\title{
Supporting Information: \\ Porphyrins Bearing Mono or Tripodal Benzylphosphonic Acid Tethers for Attachment to Oxide Surfaces
}

\author{
Robert S. Loewe, Arounaguiry Ambroise, Kannan Muthukumaran, Kisari Padmaja, \\ Andrey B. Lysenko, Guru Mathur, Qiliang Li, \\ David F. Bocian, Veena Misra, and Jonathan S. Lindsey
}

Table of Contents:

Complete Experimental Section

S2-S9

Experimental Data

S10-S66 


\section{Experimental Section}

General. ${ }^{1} \mathrm{H}(300$ or $400 \mathrm{MHz}),{ }^{13} \mathrm{C}(75$ or $100 \mathrm{MHz})$, and ${ }^{31} \mathrm{P}(121$ or $162 \mathrm{MHz}) \mathrm{NMR}$ spectra were recorded in $\mathrm{CDCl}_{3}$ unless noted otherwise. Mass spectra of compounds were obtained by FABMS, laser desorption mass spectrometry in the absence of a matrix (LDMS), ${ }^{41}$ or by MALDI-MS using the matrix 1,4-bis(5-phenyloxazol-2-yl)benzene (POPOP). Absorption and emission spectra were collected in toluene unless noted otherwise. Melting points are uncorrected. Silica gel (40 $\mu \mathrm{m}$ average particle size) and alumina (80-200 mesh) were used for column chromatography. Phosphoric acid was used as external standard (referenced to $\delta=0.00$ ppm) for ${ }^{31} \mathrm{P}$ NMR spectroscopy. $\mathrm{CHCl}_{3}$ stabilized with $0.8 \%$ ethanol was used for the porphyrin-forming reactions and chromatography procedures. $\mathrm{CHCl}_{3}$ stabilized with amylenes (not ethanol) was used for the cleavage of dialkyl phosphonates.

Non-commercial compounds. Compounds $1,{ }^{24} \mathbf{6},{ }^{28} \mathbf{1 0},,^{31} \mathbf{1 2},{ }^{33} \mathbf{1 6},,^{34,36} \mathbf{1 7},,^{37} \mathbf{1 9},{ }^{14} \mathbf{2 8}{ }^{40}$ and $\mathbf{Z n 3 1}{ }^{1}$ were prepared as described in the literature.

4-(Diethoxyphosphorylmethyl)benzoic acid (1). Triethylphosphite (9.28 mL, 53.3 mmol) and $\alpha$-bromo- $p$-toluic acid (10.4 g, $48.4 \mathrm{mmol})$ were suspended in toluene $(25 \mathrm{~mL})$ and the mixture was heated to reflux for $18 \mathrm{~h}$. The mixture was cooled to room temperature. After $12 \mathrm{~h}$, the resulting white solid was collected by suction filtration, washed with petroleum ether and dried to yield a white solid (10.2 g, 77\%): mp 117-120 ${ }^{\circ} \mathrm{C} ;{ }^{1} \mathrm{H}$ NMR $\delta 1.26(\mathrm{t}, J=6.8 \mathrm{~Hz}$, $6 \mathrm{H}), 3.24(\mathrm{~d}, J=25.6 \mathrm{~Hz}, 2 \mathrm{H}), 4.06(\mathrm{~m}, 4 \mathrm{H}), 7.38-7.40(\mathrm{~m}, 2 \mathrm{H}), 8.02(\mathrm{~d}, J=7.6 \mathrm{~Hz}, 2 \mathrm{H}), 11.4$ (br s, $1 \mathrm{H}) ;{ }^{13} \mathrm{C}$ NMR $\delta 16.5,16.6,33.4,34.8,62.8,62.9,129.1,129.2,130.00,130.05,130.10$, 130.48, 130.51, 137.3, 137.4, 170.1; FABMS obsd 273.0896, calcd 273.0892; ${ }^{31} \mathrm{P}$ NMR $\delta 26.7$; Anal. Calcd. for $\mathrm{C}_{12} \mathrm{H}_{17} \mathrm{O}_{5} \mathrm{P}: \mathrm{C}, 52.94 ; \mathrm{H}, 6.29$; Found: C, 52.92; H, 6.28.

4-(Diethoxyphosphorylmethyl)benzaldehyde (3). A suspension of 1 (9.99 g, 36.7 mmol) in anhydrous THF $(40 \mathrm{~mL})$ at $0{ }^{\circ} \mathrm{C}$ was treated dropwise over 10 min with borane-THF complex (40.3 mL, $40.3 \mathrm{mmol}, 1.0 \mathrm{M}$ in THF). The reaction mixture was stirred at room temperature for $4 \mathrm{~h}$. The reaction was quenched by slow addition of water. The mixture was concentrated and the residue was partitioned between water $(100 \mathrm{~mL})$ and $\mathrm{CH}_{2} \mathrm{Cl}_{2}(100 \mathrm{~mL})$. The aqueous layer was washed with $\mathrm{CH}_{2} \mathrm{Cl}_{2}$. The combined organic layers were washed with water, dried $\left(\mathrm{MgSO}_{4}\right)$, and concentrated to give a yellow oil. Analysis by TLC and ${ }^{1} \mathrm{H}$ NMR spectroscopy showed starting material $(\sim 10 \%)$ and the desired benzyl alcohol 2. A sample of PCC (10.8 g, $50.1 \mathrm{mmol})$ in $\mathrm{CH}_{2} \mathrm{Cl}_{2}(50 \mathrm{~mL})$ was rapidly stirred while $2(9.56 \mathrm{~g})$ in $\mathrm{CH}_{2} \mathrm{Cl}_{2}$ was rapidly added. After $2 \mathrm{~h}$, ethyl ether $(150 \mathrm{~mL})$ was added and the organic solution was decanted from the tarry residue. The residue was washed with ethyl ether $(3 \times 50 \mathrm{~mL})$. The combined organic solution was filtered through Florisil and concentrated to a brown oil. Kugelrohr distillation (190 ${ }^{\circ} \mathrm{C} @ 0.05$ Torr) afforded a clear, colorless oil $(6.54 \mathrm{~g}, 77 \%):{ }^{1} \mathrm{H}$ NMR $\delta 1.24(\mathrm{t}$, $J=7.2 \mathrm{~Hz}, 6 \mathrm{H}), 3.21(\mathrm{~d}, J=22.4 \mathrm{~Hz}, 2 \mathrm{H}), 4.03(\mathrm{~m}, 4 \mathrm{H}), 7.45-7.48(\mathrm{~m}, 2 \mathrm{H}), 7.83(\mathrm{~d}, J=7.6 \mathrm{~Hz}$, 2H), $9.99(\mathrm{~s}, 1 \mathrm{H}) ;{ }^{13} \mathrm{C}$ NMR $\delta 16.1,16.2,33.0,34.9,62.1,62.2,77.2,129.49,129.53,129.6$, 129.66, 129.71, 130.2, 130.2, 138.8, 138.9, 191.61, 191.63; ${ }^{31} \mathrm{P}$ NMR $\delta 25.4,25.9$; FABMS obsd 257.0949, calcd 257.0943 $\left(\mathrm{C}_{12} \mathrm{H}_{17} \mathrm{PO}_{4}\right)$.

5-[4-(Diethoxyphosphorylmethyl)phenyl]-10,15,20-trimesitylporphyrin (4) (Method A). Following a standard procedure for mixed-aldehyde condensation ${ }^{25}$ at high concentration ${ }^{26}$ with $\mathrm{BF}_{3} \cdot \mathrm{O}(\mathrm{Et})_{2}$-ethanol cocatalysis, ${ }^{27}$ samples of $\mathbf{3}(500 \mathrm{mg}, 1.95 \mathrm{mmol})$, mesitaldehyde (865 $\mu \mathrm{L}, 5.87 \mathrm{mmol})$, and pyrrole $(540 \mu \mathrm{L}, 7.78 \mathrm{mmol})$ were condensed in $\mathrm{CHCl}_{3}(107 \mathrm{~mL})$ in the presence of $\mathrm{BF}_{3} \cdot \mathrm{O}(\mathrm{Et})_{2}(242 \mu \mathrm{L}, 1.92 \mathrm{mmol})$ at room temperature for $1 \mathrm{~h}$. DDQ $(1.33 \mathrm{~g}, 5.86$ 
mmol) was added. After $1 \mathrm{~h}$, TEA $(267 \mu \mathrm{L}, 1.91 \mathrm{mmol})$ was added and the crude mixture was passed through a silica column $\left[\mathrm{CHCl}_{3}\right.$ /ethyl acetate (95:5)]. A second column [silica, $\mathrm{CHCl}_{3}$ /ethyl acetate (95:5)] afforded a purple solid (164 mg, 9.4\%): ${ }^{1} \mathrm{H}$ NMR $\delta-2.57$ (br s, 2H), $1.42(\mathrm{t}, J=6.8 \mathrm{~Hz}, 6 \mathrm{H}), 1.85-1.86(\mathrm{~m}, 18 \mathrm{H}), 2.63(\mathrm{~s}, 9 \mathrm{H}), 3.50(\mathrm{~d}, J=22.0 \mathrm{~Hz}, 2 \mathrm{H}), 4.23(\mathrm{p}, J=$ $6.8 \mathrm{~Hz}, 4 \mathrm{H}), 7.28(\mathrm{~s}, 6 \mathrm{H}), 7.68\left(\mathrm{dd}, J^{1}=7.6 \mathrm{~Hz}, J^{2}=2.4 \mathrm{~Hz}, 2 \mathrm{H}\right), 8.15(\mathrm{~d}, J=7.6 \mathrm{~Hz}, 2 \mathrm{H}), 8.63$ $(\mathrm{s}, 4 \mathrm{H}), 8.67(\mathrm{~d}, J=4.0 \mathrm{~Hz}, 2 \mathrm{H}), 8.75(\mathrm{~d}, J=4.0 \mathrm{~Hz}, 2 \mathrm{H}) ;{ }^{31} \mathrm{P}$ NMR $\delta 27.1$; LDMS obsd 890.6; FABMS obsd 890.4351; calcd $890.4325\left(\mathrm{C}_{58} \mathrm{H}_{59} \mathrm{~N}_{4} \mathrm{O}_{3} \mathrm{P}\right) . \lambda_{\mathrm{abs}}=419,515,547,592,649 \mathrm{~nm} ; \lambda_{\mathrm{em}}$ $\left(\lambda_{\mathrm{ex}}=515 \mathrm{~nm}\right) 650720 \mathrm{~nm}$.

(Method B). A solution of $7(200 \mathrm{mg}, 240 \mu \mathrm{mol})$ in triethylphosphite $(2.1 \mathrm{~mL})$ and toluene $(8 \mathrm{~mL})$ was heated to $120^{\circ} \mathrm{C}$ under argon for $24 \mathrm{~h}$. The reaction mixture was cooled and concentrated under vacuum (to remove excess triethylphosphite; $b p=154{ }^{\circ} \mathrm{C}$ ). Column chromatography [silica, $\mathrm{CHCl}_{3} \rightarrow \mathrm{CHCl}_{3}$ /ethyl acetate (95:5)] afforded a purple solid (165 mg, $80 \%)$. Characterization data were consistent with those described above.

(Method C). A solution of diethylphosphite $(1.16 \mathrm{~mL}, 9.00 \mathrm{mmol})$ in THF $(20 \mathrm{~mL})$ was treated with $\mathrm{NaH}$ (206 mg, $8.60 \mathrm{mmol}$ ) and the mixture was stirred at room temperature under $\operatorname{argon}$ for $1 \mathrm{~h}$. A $1.9 \mathrm{~mL}$ aliquot of this solution $(864 \mathrm{mmol}, 3 \mathrm{eq})$ was then added to a solution of $7(240 \mathrm{mg}, 288 \mu \mathrm{mol})$ in THF $(20 \mathrm{~mL})$. The reaction mixture was stirred at under argon for $24 \mathrm{~h}$. Water and $\mathrm{CH}_{2} \mathrm{Cl}_{2}$ were added. The layers were separated and the aqueous layer was washed with $\mathrm{CH}_{2} \mathrm{Cl}_{2}$. The organic layers were combined, dried $\left(\mathrm{K}_{2} \mathrm{CO}_{3}\right)$, and concentrated. Column chromatography [silica, $\mathrm{CHCl}_{3} \rightarrow \mathrm{CHCl}_{3}$ /ethyl acetate (95:5)] afforded a purple solid (187 $\mathrm{mg}$, $73 \%$ ). Characterization data were consistent with those described above.

\section{Zn(II)-5-[4-(Diethoxyphosphorylmethyl)phenyl]-10,15,20-trimesitylporphyrin}

(Zn4). A solution of $4(163 \mathrm{mg}, 189 \mu \mathrm{mol})$ in $\mathrm{CHCl}_{3}(15 \mathrm{~mL})$ was treated with a solution of $\mathrm{Zn}(\mathrm{OAc})_{2} \cdot 2 \mathrm{H}_{2} \mathrm{O}(207 \mathrm{mg}, 943 \mu \mathrm{mol})$ in methanol $(3 \mathrm{~mL})$. Column chromatography [silica, $\mathrm{CHCl}_{3}$ /ethyl acetate (95:5)] afforded a purple solid (164 mg, 94\%): ${ }^{1} \mathrm{H}$ NMR $\left(\mathrm{THF}-\mathrm{d}_{8}\right) \delta 1.35(\mathrm{t}$, $J=6.9 \mathrm{~Hz}, 6 \mathrm{H}), 1.86(\mathrm{~s}, 18 \mathrm{H}), 2.61(\mathrm{~s}, 9 \mathrm{H}), 3.46(\mathrm{~d}, J=22.0 \mathrm{~Hz}, 2 \mathrm{H}), 4.14(\mathrm{p}, J=7.2 \mathrm{~Hz}, 4 \mathrm{H})$, $7.29(\mathrm{~s}, 6 \mathrm{H}), 7.70\left(\mathrm{dd}, J^{1}=7.5 \mathrm{~Hz}, J^{2}=1.8 \mathrm{~Hz}, 2 \mathrm{H}\right), 8.11(\mathrm{~d}, J=7.5 \mathrm{~Hz}, 2 \mathrm{H}), 8.62-8.63(\mathrm{~m}, 6 \mathrm{H})$, $8.76(\mathrm{~d}, J=4.5 \mathrm{~Hz}, 2 \mathrm{H}) ;{ }^{31} \mathrm{P}$ NMR $\delta 26.8$; LDMS obsd 956.0; FABMS obsd 952.3466; calcd $952.3460\left(\mathrm{C}_{58} \mathrm{H}_{57} \mathrm{~N}_{4} \mathrm{O}_{3} \mathrm{PZn}\right) ; \lambda_{\mathrm{abs}}=425,555,595 \mathrm{~nm}$.

\section{Zn(II)-5-[4-(Dihydroxyphosphorylmethyl)phenyl]-10,15,20-trimesitylporphyrin}

(Zn5) (from Zn4). A mixture of $\mathbf{Z n 4}(178 \mathrm{mg}, 186 \mu \mathrm{mol})$ in $\mathrm{CHCl}_{3}(18 \mathrm{~mL})$ and TEA $(518 \mu \mathrm{L}$, $3.72 \mathrm{mmol}$ ) was heated to reflux, affording a homogeneous solution. A sample of TMS-Br (369 $\mu \mathrm{L}, 2.80 \mathrm{mmol}$ ) was then added. The solution was stirred at reflux for $4 \mathrm{~h}$, then cooled. The reaction mixture was washed with water. The organic layer was collected, dried $\left(\mathrm{Na}_{2} \mathrm{SO}_{4}\right)$, and concentrated. Column chromatography [silica, $\mathrm{CHCl}_{3} / \mathrm{MeOH}(1: 1)$ ] afforded a purple solid. The solid was washed with hexanes, then water, then hexanes again to afford a purple solid (130 $\mathrm{mg}$, 78\%): ${ }^{1} \mathrm{H}$ NMR $\left(\mathrm{DMSO}_{6}\right) \delta 1.71(\mathrm{~s}, 9 \mathrm{H}), 1.76(\mathrm{~s}, 9 \mathrm{H}), 2.54(\mathrm{~s}, 3 \mathrm{H})$, the other methyl signal is presumed to be buried under the DMSO solvent peak, $7.20(\mathrm{~s}, 3 \mathrm{H}), 7.36(\mathrm{~s}, 3 \mathrm{H}), 7.69(\mathrm{~d}, 2 \mathrm{H})$, $7.97(\mathrm{~d}, 2 \mathrm{H}), 8.47(\mathrm{~s}, 6 \mathrm{H}), 8.74(\mathrm{~s}, 2 \mathrm{H}) ;{ }^{31} \mathrm{P}$ NMR analysis was attempted but no peak could be observed; LDMS obsd 927.7 [(M + OMe $\left.)^{+}\right]$; FABMS obsd 896.2870; calcd 896.2834 $\left(\mathrm{C}_{48} \mathrm{H}_{49} \mathrm{~N}_{4} \mathrm{O}_{3} \mathrm{PZn}\right) ; \lambda_{\text {abs }}=426,556,596 \mathrm{~nm} ; \lambda_{\text {em }}\left(\lambda_{\text {ex }}=556 \mathrm{~nm}\right) 601,655 \mathrm{~nm}$.

\section{Zn(II)-5-[4-(Dihydroxyphosphorylmethyl)phenyl]-10,15,20-trimesitylporphyrin}

(Zn5) (from Zn8). A mixture of $\mathbf{Z n 8}(159 \mathrm{mg}, 172 \mu \mathrm{mol})$ in $\mathrm{CHCl}_{3}(17 \mathrm{~mL})$ and TEA (479 $\mu \mathrm{L}$, $3.44 \mathrm{mmol})$ was heated to reflux, affording a homogeneous solution. A sample of TMS-Br (341 
$\mu \mathrm{L}, 2.58 \mathrm{mmol}$ ) was then added. The solution was stirred at reflux for $2 \mathrm{~h}$ then cooled. Analogous workup as described above yielded a purple solid (120 mg, 77\%). Characterization data were consistent with those described above.

5-[4-(Bromomethyl)phenyl]-10,15,20-trimesitylporphyrin (7). Following a standard procedure for mixed-aldehyde condensation ${ }^{25}$ at high concentration ${ }^{26}$ with $\mathrm{BF}_{3} \cdot \mathrm{O}(\mathrm{Et})_{2}$-ethanol cocatalysis, ${ }^{27}$ samples of $6(700 \mathrm{mg}, 3.52 \mathrm{mmol})$, mesitaldehyde $(1.56 \mathrm{~mL}, 10.6 \mathrm{mmol})$, and pyrrole $(977 \mu \mathrm{L}, 14.1 \mathrm{mmol})$ were condensed in $\mathrm{CHCl}_{3}(193 \mathrm{~mL})$ in the presence of $\mathrm{BF}_{3} \cdot \mathrm{O}(\mathrm{Et})_{2}$ $(435 \mu \mathrm{L}, 3.43 \mathrm{mmol})$ at room temperature for $1 \mathrm{~h}$. DDQ $(2.41 \mathrm{~g}, 10.6 \mathrm{mmol})$ was added. After 1 h, TEA (478 $\mu \mathrm{mol}, 3.43 \mathrm{mmol})$ was added and the crude mixture was passed over a pad of silica $\left[\mathrm{CH}_{2} \mathrm{Cl}_{2} /\right.$ hexanes (1:1)] followed by column chromatography [silica, hexanes/ $\mathrm{CH}_{2} \mathrm{Cl}_{2}(7: 3), 10 \mathrm{x}$ $30 \mathrm{~cm}]$ to afford a purple solid $(465 \mathrm{mg}, 16 \%):{ }^{1} \mathrm{H}$ NMR $\delta-2.57(\mathrm{br} \mathrm{s}, 2 \mathrm{H}), 1.86(\mathrm{~s}, 18 \mathrm{H}), 2.63$ $(\mathrm{s}, 9 \mathrm{H}), 4.85$ (s, 2H), $7.28(\mathrm{~s}, 6 \mathrm{H}), 7.77(\mathrm{~d}, J=7.2 \mathrm{~Hz}, 2 \mathrm{H}), 8.18(\mathrm{~d}, J=7.2 \mathrm{~Hz}, 2 \mathrm{H}), 8.64(\mathrm{~s}, 4 \mathrm{H})$, $8.68(\mathrm{~d}, J=4.4 \mathrm{~Hz}, 2 \mathrm{H}), 8.77(\mathrm{~d}, J=4.4 \mathrm{~Hz}, 2 \mathrm{H})$; LDMS obsd 830.4, $751.5\left[(\mathrm{M}-\mathrm{Br})^{+}\right]$. FABMS obsd 832.3135; calcd 832.3141 $\left(\mathrm{C}_{54} \mathrm{H}_{49} \mathrm{BrN}_{4}\right) ; \lambda_{\text {abs }}=420,515,547,592,649 \mathrm{~nm} ; \lambda_{\mathrm{em}}$ $\left(\lambda_{\text {ex }}=515 \mathrm{~nm}\right) 650,720 \mathrm{~nm}$.

5-[4-(Dimethoxyphosphorylmethyl)phenyl]-10,15,20-trimesitylporphyrin (8). A solution of $7(200 \mathrm{mg}, 240 \mu \mathrm{mol})$ in trimethylphosphite $(2.2 \mathrm{~mL})$ and toluene $(5 \mathrm{~mL})$ was heated to $120{ }^{\circ} \mathrm{C}$ under argon for $17 \mathrm{~h}$. The reaction mixture was cooled and concentrated under vacuum. The residue was chromatographed [silica, $\mathrm{CHCl}_{3} \rightarrow \mathrm{CHCl}_{3}$ /ethyl acetate (95:5)] to afford a purple solid (164 mg, 79\%): ${ }^{1} \mathrm{H}$ NMR $\delta-2.57$ (br s, 2H), 1.84-1.85 (m, 18H), 2.62 (s, $9 \mathrm{H}), 3.50(\mathrm{~d}, J=21.6 \mathrm{~Hz}, 2 \mathrm{H}), 3.87(\mathrm{~d}, J=11.1 \mathrm{~Hz}, 6 \mathrm{H}), 7.27(\mathrm{~s}, 6 \mathrm{H}), 7.67\left(\mathrm{dd}, J^{1}=8.4 \mathrm{~Hz}, J^{2}=\right.$ $1.8 \mathrm{~Hz}, 2 \mathrm{H}), 8.15(\mathrm{~d}, J=7.8 \mathrm{~Hz}, 2 \mathrm{H}), 8.63(\mathrm{~s}, 4 \mathrm{H}), 8.67$ (d, $J=4.8 \mathrm{~Hz}, 2 \mathrm{H}), 8.75$ (d, $J=4.8 \mathrm{~Hz}$, $2 \mathrm{H}) ;{ }^{31} \mathrm{P}$ NMR $\delta 29.50$; LDMS obsd 863.2; FABMS obsd 862.4045; calcd 862.4012 $\left(\mathrm{C}_{56} \mathrm{H}_{55} \mathrm{~N}_{4} \mathrm{O}_{3} \mathrm{P}\right) ; \lambda_{\text {abs }}=421,514,547,593,649 \mathrm{~nm}$.

\section{Zn(II)-5-[4-(Dimethoxyphosphorylmethyl)phenyl]-10,15,20-trimesitylporphyrin}

(Zn8). A solution of $8(160 \mathrm{mg}, 185 \mu \mathrm{mol})$ in $\mathrm{CHCl}_{3}(12 \mathrm{~mL})$ was treated with a solution of $\mathrm{Zn}(\mathrm{OAc})_{2} \cdot 2 \mathrm{H}_{2} \mathrm{O}(204 \mathrm{mg}, 0.929 \mathrm{mmol})$ in methanol $(3 \mathrm{~mL})$. Column chromatography [silica, $\mathrm{CHCl}_{3}$ /ethyl acetate (95:5)] afforded a purple solid (169 mg, 98\%): ${ }^{1} \mathrm{H}$ NMR $\left(\mathrm{THF}-\mathrm{d}_{8}\right) \delta 1.85(\mathrm{~s}$, $18 \mathrm{H}), 2.60(\mathrm{~s}, 9 \mathrm{H}), 3.47(\mathrm{~d}, J=22.0 \mathrm{~Hz}, 2 \mathrm{H}), 3.77(\mathrm{~d}, J=11.1 \mathrm{~Hz}, 6 \mathrm{H}), 7.28(\mathrm{~s}, 6 \mathrm{H}), 7.69(\mathrm{~d}, J=$ $7.5 \mathrm{~Hz}, 2 \mathrm{H}), 8.11(\mathrm{~d}, J=7.6 \mathrm{~Hz}, 2 \mathrm{H}), 8.61-8.63(\mathrm{~m}, 6 \mathrm{H}), 8.75(\mathrm{~d}, J=4.5 \mathrm{~Hz}, 2 \mathrm{H}) ;{ }^{31} \mathrm{P}$ NMR $\delta$ 29.2; LDMS obsd 926.1; FABMS obsd 924.3146; calcd $924.3147\left(\mathrm{C}_{56} \mathrm{H}_{53} \mathrm{~N}_{4} \mathrm{O}_{3} \mathrm{PZn}\right)$; $\lambda_{\text {abs }}=$ $423,551,592 \mathrm{~nm}$.

5-[4-(Diethoxyphosphorylmethyl)phenyl]dipyrromethane (9). Following a standard procedure, ${ }^{37}$ a solution of $\mathbf{3}(2.00 \mathrm{~g}, 7.81 \mathrm{mmol})$ in pyrrole $(22.0 \mathrm{~mL}, 315 \mathrm{mmol})$ was treated with TFA $(60 \mu \mathrm{L}, 780 \mu \mathrm{mol})$ at room temperature under argon for $5 \mathrm{~min}$. TEA $(109 \mu \mathrm{L}, 782$ $\mu \mathrm{mol})$ was added and the reaction mixture was concentrated under vacuum. Column chromatography (silica, ethyl acetate) afforded a viscous, yellow oil $(1.33 \mathrm{~g}, 46 \%)$ : ${ }^{1} \mathrm{H}$ NMR $\left(\mathrm{CD}_{2} \mathrm{Cl}_{2}\right) \delta 1.25(\mathrm{t}, J=6.4 \mathrm{~Hz}, 6 \mathrm{H}), 3.11(\mathrm{~d}, J=21.6 \mathrm{~Hz}, 2 \mathrm{H}), 4.01(\mathrm{~m}, 4 \mathrm{H}), 5.43(\mathrm{~s}, 1 \mathrm{H}), 5.86(\mathrm{~s}$, $2 \mathrm{H}), 6.11(\mathrm{~d}, J=2.8 \mathrm{~Hz}, 2 \mathrm{H}), 6.67(\mathrm{~s}, 2 \mathrm{H}), 7.14(\mathrm{~d}, J=8.0 \mathrm{~Hz}, 2 \mathrm{H}), 7.23(\mathrm{~d}, J=8.0 \mathrm{~Hz}, 2 \mathrm{H})$, 8.29 (br s, 2H); ${ }^{13} \mathrm{C}$ NMR $\delta 16.6,16.7,32.5,34.3,43.74,43.75,62.35,62.44,107.3,108.3$, $117.5,128.8,128.9,130.06,130.14,130.2,132.81,132.83,141.39,141.44 ;{ }^{31} \mathrm{P}$ NMR $\delta 27.6$; FABMS obsd 372.1598, calcd $372.1603\left(\mathrm{C}_{20} \mathrm{H}_{25} \mathrm{~N}_{2} \mathrm{O}_{3} \mathrm{P}\right)$.

5-[4-(Diethoxyphosphorylmethyl)phenyl]-10,15,20-tri-p-tolylporphyrin (11).

Following a standard procedure, ${ }^{32}$ a solution of $\mathbf{1 0}(360 \mathrm{mg}, 762 \mu \mathrm{mol})$ in dry THF/methanol [44 
mL, (10:1)] was treated with $\mathrm{NaBH}_{4}(576 \mathrm{mg}, 15.2 \mathrm{mmol})$. After $40 \mathrm{~min}$, the reaction mixture was poured into a stirred mixture of saturated aqueous $\mathrm{NH}_{4} \mathrm{Cl}(150 \mathrm{~mL})$ and $\mathrm{CH}_{2} \mathrm{Cl}_{2}(150 \mathrm{~mL})$. The organic phase was washed with water and brine, dried $\left(\mathrm{Na}_{2} \mathrm{SO}_{4}\right)$, and concentrated. The resulting 10-diol was dissolved in $\mathrm{CH}_{2} \mathrm{Cl}_{2}(305 \mathrm{~mL})$. The solution was treated with 9 (284 mg, $762 \mu \mathrm{mol})$ and $\mathrm{InCl}_{3}(22 \mathrm{mg}, 99 \mu \mathrm{mol})$ under argon for $1 \mathrm{~h}$. DDQ (519 $\left.\mathrm{mg}, 2.29 \mathrm{mmol}\right)$ was added and the mixture was stirred for $1 \mathrm{~h}$ at room temperature. TEA $(1 \mathrm{~mL})$ was added and the reaction mixture was chromatographed [silica, $\mathrm{CHCl}_{3}$ /ethyl acetate (9:1); silica, $\mathrm{CHCl}_{3} /$ ethyl acetate (95:5)] to afford a purple solid. The solid was dissolved in $\mathrm{CHCl}_{3}(120 \mathrm{~mL})$ and treated overnight with a solution of $\mathrm{Zn}(\mathrm{OAc})_{2} \cdot 2 \mathrm{H}_{2} \mathrm{O}(220 \mathrm{mg}, 1.00 \mathrm{mmol})$ in methanol $(12 \mathrm{~mL})$ at room temperature. The reaction mixture was concentrated and chromatographed [silica, $\mathrm{CHCl}_{3} /$ ethyl acetate (4:1)] to afford a purple solid $(95 \mathrm{mg})$. This compound could not be analyzed for purity due to its poor solubility. The solid was suspended $(95 \mathrm{mg}, 110 \mu \mathrm{mol})$ in $\mathrm{CH}_{2} \mathrm{Cl}_{2}(80 \mathrm{~mL})$ and treated with TFA (420 $\mu \mathrm{L}, 5.5 \mathrm{mmol}, 50 \mathrm{eq})$ at room temperature under argon for $1 \mathrm{~h}$. The solution was washed with $10 \%$ aqueous $\mathrm{NaHCO}_{3}$ and water. The organic layer was dried $\left(\mathrm{Na}_{2} \mathrm{SO}_{4}\right)$, filtered, and concentrated. Column chromatography [silica, $\mathrm{CHCl}_{3}$ /ethyl acetate (1:1)] afforded a purple solid $(82 \mathrm{mg}, 12 \%)$ : ${ }^{1} \mathrm{H}$ NMR $\delta-2.78(\mathrm{br} \mathrm{s}, 2 \mathrm{H}), 1.41(\mathrm{t}, J=6.9 \mathrm{~Hz}, 6 \mathrm{H}), 2.71$ $(\mathrm{s}, 9 \mathrm{H}), 3.50(\mathrm{~d}, J=21.6 \mathrm{~Hz}, 2 \mathrm{H}), 4.22(\mathrm{p}, J=6.9 \mathrm{~Hz}, 4 \mathrm{H}), 7.55(\mathrm{~d}, J=7.8 \mathrm{~Hz}, 6 \mathrm{H}), 7.69(\mathrm{~d}, J=$ $7.2 \mathrm{~Hz}, 2 \mathrm{H}), 8.10(\mathrm{~d}, J=7.8 \mathrm{~Hz}, 6 \mathrm{H}), 8.16(\mathrm{~d}, J=7.2 \mathrm{~Hz}, 2 \mathrm{H}), 8.80-8.86(\mathrm{~m}, 8 \mathrm{H}) ;{ }^{31} \mathrm{P}$ NMR $\delta$ 27.1; LDMS obsd 805.2; FABMS obsd 806.3401, calcd $806.3386\left(\mathrm{C}_{52} \mathrm{H}_{47} \mathrm{~N}_{4} \mathrm{O}_{3} \mathrm{P}\right) ; \lambda_{\text {abs }}=421$, $516,551,593,649 \mathrm{~nm}$.

5-(6-Bromohexyl)-10,15,20-tri-p-tolylporphyrin (13). Following a standard procedure, ${ }^{32}$ a solution of $\mathbf{1 0}(236 \mathrm{mg}, 0.500 \mathrm{mmol})$ in dry $\mathrm{THF} / \mathrm{CH}_{3} \mathrm{OH}$ [22 $\left.\mathrm{mL},(10: 1)\right]$ was treated with $\mathrm{NaBH}_{4}(378 \mathrm{mg}, 10.0 \mathrm{mmol})$. After $40 \mathrm{~min}$, the reaction mixture was poured into a stirred mixture of aqueous $\mathrm{NH}_{4} \mathrm{Cl}(100 \mathrm{~mL})$ and $\mathrm{CH}_{2} \mathrm{Cl}_{2}(100 \mathrm{~mL})$. The organic phase was isolated, washed with water, dried $\left(\mathrm{Na}_{2} \mathrm{SO}_{4}\right)$, and concentrated. The resulting 10-diol was dissolved in $\mathrm{CH}_{2} \mathrm{Cl}_{2}(200 \mathrm{~mL})$. The solution was treated with $12(153 \mathrm{mg}, 0.500 \mathrm{mmol})$ and $\mathrm{InCl}_{3}(14.2 \mathrm{mg}, 63.7 \mu \mathrm{mol})$ under argon for $40 \mathrm{~min}$. DDQ (341 mg, $\left.1.50 \mathrm{mmol}\right)$ was added and the mixture was stirred at room temperature for $1 \mathrm{~h}$. TEA $(3 \mathrm{~mL})$ was added and the mixture was concentrated and filtered [silica, hexanes $/ \mathrm{CH}_{2} \mathrm{Cl}_{2}$ (7:3)]. The porphyrin band was collected and recrystallized $\left(\mathrm{CH}_{2} \mathrm{Cl}_{2} / \mathrm{CH}_{3} \mathrm{OH}\right)$ to afford a purple solid $(88 \mathrm{mg}, 24 \%)$ : ${ }^{1} \mathrm{H} \mathrm{NMR} \delta-2.72(\mathrm{~s}, 2 \mathrm{H})$, 1.60-1.70 (m, 2H), 1.75-1.84 (m, 2H), 1.85-1.96 (m, 2H), 2.52-2.62 (m, 2H), $2.71(\mathrm{~s}, 3 \mathrm{H}), 2.73$ (s, 6H), 3.38-3.44 (m, 2H), 5.00-5.07 (m, 2H), 7.52-7.59 (m, 6H), 8.05-8.12 (m, 6H), 8.82 (s, $4 \mathrm{H}), 8.95(\mathrm{~d}, J=5.12 \mathrm{H}), 9.47(\mathrm{~d}, J=5.1 \mathrm{~Hz}, 2 \mathrm{H})$; LDMS obsd 743.8; FABMS obsd 742.2653, calcd $742.2671\left(\mathrm{C}_{47} \mathrm{H}_{43} \mathrm{BrN}_{4}\right) ; \lambda_{\text {abs }}=418,516,550,594,651 \mathrm{~nm}$.

Zn(II)-5-(6-Bromohexyl)-5,10,15-tri-p-tolylporphyrin (Zn13). A solution of 13 (74.4 $\mathrm{mg}, 0.100 \mathrm{mmol})$ in $\mathrm{THF} / \mathrm{CH}_{2} \mathrm{Cl}_{2}[20 \mathrm{~mL},(1: 1)]$ was treated with $\mathrm{Zn}(\mathrm{OAc})_{2} \cdot 2 \mathrm{H}_{2} \mathrm{O}(109 \mathrm{mg}$, $0.500 \mathrm{mmol}$ ). After $1 \mathrm{~h}$, the mixture was concentrated and chromatographed [silica, hexanes/toluene $(1: 1)]$ to afford a purple solid $(81 \mathrm{mg}, 85 \%):{ }^{1} \mathrm{H}$ NMR $\delta 1.60-1.70(\mathrm{~m}, 2 \mathrm{H})$, 1.80-1.96 (m, 4H), 2.52-2.62 (m, 2H), $2.67(\mathrm{~s}, 3 \mathrm{H}), 2.70(\mathrm{~s}, 6 \mathrm{H}), 3.45(\mathrm{t}, J=6.8 \mathrm{~Hz}, 2 \mathrm{H}), 5.10-$ $5.18(\mathrm{~m}, 2 \mathrm{H}), 7.50-7.58(\mathrm{~m}, 6 \mathrm{H}), 8.01-8.08(\mathrm{~m}, 6 \mathrm{H}), 8.76-8.80(\mathrm{~m}, 4 \mathrm{H}), 8.91(\mathrm{~d}, J=4.8 \mathrm{~Hz}$, 2H), $9.60(\mathrm{~d}, J=4.8 \mathrm{~Hz}, 2 \mathrm{H})$; LDMS obsd 806.9; FABMS obsd 804.1825, calcd 804.1806 $\left(\mathrm{C}_{47} \mathrm{H}_{41} \mathrm{BrN}_{4} \mathrm{Zn}\right) ; \lambda_{\text {abs }}=424,553,591 \mathrm{~nm}$.

Zn(II)-5-[6-(Diethoxyphosphoryl)hexyl]-10,15,20-tri-p-tolylporphyrin (Zn14). A solution of Zn13 (40.4 mg, $50.1 \mu \mathrm{mol})$ in triethylphosphite $(2 \mathrm{~mL})$ was refluxed under argon for $2 \mathrm{~d}$. The mixture was chromatographed [silica, $\mathrm{CH}_{2} \mathrm{Cl}_{2} \rightarrow \mathrm{CH}_{2} \mathrm{Cl}_{2} / \mathrm{THF}$ (95:5)] and the second 
red fraction was collected, affording a purple solid $(42.1 \mathrm{mg}, 100 \%):{ }^{1} \mathrm{H}$ NMR $\delta 1.67(\mathrm{t}, J=7.0$ $\mathrm{Hz}, 6 \mathrm{H}), 1.48-1.68(\mathrm{~m}, 6 \mathrm{H}), 1.77-1.90(\mathrm{~m}, 2 \mathrm{H}), 2.46-2.62(\mathrm{~m}, 2 \mathrm{H}), 2.67(\mathrm{~s}, 3 \mathrm{H}), 2.70(\mathrm{~s}, 6 \mathrm{H})$, 3.80-3.96 (m, 4H), 5.10-5.18 (m, 2H), 7.51-7.58 (m, 6H), 8.01-8.08 (m, 6H), 8.75-8.80 (m, $4 \mathrm{H}), 8.91(\mathrm{~d}, J=4.8 \mathrm{~Hz}, 2 \mathrm{H}), 9.60(\mathrm{~d}, J=4.8 \mathrm{~Hz}, 2 \mathrm{H}) ;{ }^{31} \mathrm{P}$ NMR $\delta 31.9$; LDMS obsd 864.9; FABMS obsd 862.3003, calcd 862.2990 $\left(\mathrm{C}_{51} \mathrm{H}_{51} \mathrm{~N}_{4} \mathrm{O}_{3} \mathrm{PZn}\right) ; \lambda_{\text {abs }}=424,558,599 \mathrm{~nm}$.

Zn(II)-5-[6-(Dihydroxyphosphoryl)hexyl]-10,15,20-tri-p-tolylporphyrin (Zn15). A solution of $\mathbf{Z n 1 4}(43.2 \mathrm{mg}, 50.0 \mu \mathrm{mol})$ in $\mathrm{CHCl}_{3}(15 \mathrm{~mL})$ was treated with TEA $(140 \mu \mathrm{L}, 1.0$ mmol) followed by TMS-Br $(99.0 \mu \mathrm{L}, 0.75 \mathrm{mmol})$. The mixture was stirred overnight at 60-65 ${ }^{\circ} \mathrm{C}$ under argon. The reaction mixture was cooled and water and $\mathrm{CH}_{2} \mathrm{Cl}_{2}$ were added. The organic layer was separated, washed with water, dried $\left(\mathrm{Na}_{2} \mathrm{SO}_{4}\right)$ and concentrated. Recrystallization $\left(\mathrm{CH}_{2} \mathrm{Cl}_{2}\right.$ /hexanes) afforded a purple solid $(35.4 \mathrm{mg}, 88 \%)$ : ${ }^{1} \mathrm{H} \mathrm{NMR} \delta 0.60-$ 1.78 (br m, 4H), 2.16-2.36 (m, 4H), 2.40-2.50 (m, 2H), 2.62 (s, 6H), 2.64 (s, 3H), 4.98-5.05 (m, 2H), 7.45-7.51 (m, 6H), 7.98-8.03 (m, 6H), 8.75 (s, 4H), 8.83-8.87 (m, 2H), 9.49-9.53 (m, 2H); ${ }^{31} \mathrm{P}$ NMR $\delta$ 27.3; LDMS obsd 809.1; FABMS obsd 806.2392, calcd 806.2364 $\left(\mathrm{C}_{47} \mathrm{H}_{43} \mathrm{~N}_{4} \mathrm{O}_{3} \mathrm{PZn}\right)$; $\lambda_{\text {abs }}=424,558,600 \mathrm{~nm}$.

\section{5-(4-Ferrocenylphenyl)-15-[4-(dimethoxyphosphorylmethyl)phenyl]-10,20-}

dimesitylporphyrin (18). A solution containing 16 (375 mg, $1.29 \mathrm{mmol}), 6$ (257 mg, 1.29 $\mathrm{mmol})$, and 17 (683 $\mathrm{mg}, 2.58 \mathrm{mmol})$ in $\mathrm{CH}_{2} \mathrm{Cl}_{2}(258 \mathrm{~mL})$ was treated with TFA $(354 \mu \mathrm{L}, 4.59$ mmol) at room temperature under argon for $1 \mathrm{~h}$. DIEA $(800 \mu \mathrm{L}, 4.59 \mathrm{mmol})$ and $p$-chloranil (952 $\mathrm{mg}, 3.87 \mathrm{mmol}$ ) were then added and the mixture was stirred for $18 \mathrm{~h}$. Column chromatography [silica, $\mathrm{CH}_{2} \mathrm{Cl}_{2}$ ] afforded a mixture of porphyrins free from polar byproducts $(553 \mathrm{mg}, 44 \%)$. The porphyrin mixture $(553 \mathrm{mg})$ was taken up in toluene $(50 \mathrm{~mL})$ and treated with trimethylphosphite $(3.4 \mathrm{~mL}, 28.8 \mathrm{mmol}, 50 \mathrm{eq})$ at reflux under argon for $2 \mathrm{~d}$. Column chromatography $\left[\mathrm{CH}_{2} \mathrm{Cl}_{2}\right.$ /ethyl acetate (3:1)] afforded a purple solid (253 mg, 20\%): ${ }^{1} \mathrm{H}$ NMR $\left(\mathrm{THF}_{\mathrm{d}}\right)_{8} \delta-2.57(\mathrm{br} \mathrm{s}, 2 \mathrm{H}), 1.85(\mathrm{~s}, 12 \mathrm{H}), 2.61(\mathrm{~s}, 6 \mathrm{H}), 3.48(\mathrm{~d}, J=21.6 \mathrm{~Hz}, 2 \mathrm{H}), 3.76(\mathrm{~d}, J=$ $10.4 \mathrm{~Hz}, 6 \mathrm{H}), 4.20(\mathrm{~s}, 5 \mathrm{H}), 4.45(\mathrm{~s}, 2 \mathrm{H}), 4.99(\mathrm{~s}, 2 \mathrm{H}), 7.30(\mathrm{~s}, 4 \mathrm{H}), 7.70\left(\mathrm{dd}, J^{1}=8.0 \mathrm{~Hz}, J^{2}=1.7\right.$ $\mathrm{Hz}, 2 \mathrm{H}), 7.89$ (d, $J=8.0 \mathrm{~Hz}, 2 \mathrm{H}), 8.11-8.14(\mathrm{~m}, 4 \mathrm{H}), 8.65(\mathrm{~d}, J=4.4 \mathrm{~Hz}, 2 \mathrm{H}), 8.67(\mathrm{~d}, J=4.4$ $\mathrm{Hz}, 2 \mathrm{H}), 8.78(\mathrm{~d}, J=4.4 \mathrm{~Hz}, 2 \mathrm{H}), 8.88(\mathrm{~d}, J=4.4 \mathrm{~Hz}, 2 \mathrm{H})$; FABMS obsd 1004.3546; calcd $1004.3518\left(\mathrm{C}_{63} \mathrm{H}_{57} \mathrm{FeN}_{4} \mathrm{O}_{3} \mathrm{P}\right) ; \lambda_{\text {abs }}=425,518,556,599 \mathrm{~nm}$.

\section{Co(II)-5-[4-(Diethoxyphosphorylmethyl)phenyl]-10,15,20-trimesitylporphyrin}

(Co4). A solution of $4(184 \mathrm{mg}, 206 \mu \mathrm{mol})$ in $\mathrm{CHCl}_{3}(100 \mathrm{~mL})$ was treated with a suspension of $\mathrm{Co}(\mathrm{OAc})_{2}(365 \mathrm{mg}, 2.06 \mathrm{mmol})$ in methanol $(20 \mathrm{~mL})$. The mixture was heated to reflux under argon. After $12 \mathrm{~h}$, the mixture was cooled and washed with water. The organic layer was dried $\left(\mathrm{K}_{2} \mathrm{CO}_{3}\right)$, filtered, and concentrated. Column chromatography [silica, $\mathrm{CHCl}_{3} /$ ethyl acetate (9:1)] afforded a red solid $(133 \mathrm{mg}, 68 \%)$ : ${ }^{1} \mathrm{H}$ NMR $\delta 2.23(\mathrm{t}, J=6.3 \mathrm{~Hz}, 6 \mathrm{H}), 3.28$ (br s, 6H), $3.58(\mathrm{br}$ $\mathrm{s}, 12 \mathrm{H}), 3.93(\mathrm{~s}, 3 \mathrm{H}), 4.01(\mathrm{~s}, 6 \mathrm{H}), 4.81(\mathrm{~d}, J=21.6 \mathrm{~Hz}, 2 \mathrm{H}), 5.12(\mathrm{~m}, 4 \mathrm{H}), 9.17(\mathrm{~s}, 2 \mathrm{H}), 9.30(\mathrm{~s}$, 4H), 9.66 (s, 2H), 12.49 (br s, 2H), 15.25 (br s, 4H), 15.50 (br s, 4H); LDMS obsd 948.9. FABMS obsd 947.3525, calcd $947.3500\left(\mathrm{C}_{58} \mathrm{H}_{57} \mathrm{CoN}_{4} \mathrm{O}_{3} \mathrm{P}\right) ; \lambda_{\text {abs }}=412,528 \mathrm{~nm}$.

\section{Co(II)-5-[4-(Dihydroxyphosphorylmethyl)phenyl]-10,15,20-trimesitylporphyrin}

(Co5). A solution of $\mathbf{C o 4}(126 \mathrm{mg}, 133 \mu \mathrm{mol})$ in $\mathrm{CHCl}_{3}(13 \mathrm{~mL})$ was treated with samples of TEA $(371 \mu \mathrm{L}, 2.66 \mathrm{mmol})$ and TMS-Br $(263 \mu \mathrm{L}, 2.00 \mathrm{mmol})$ at reflux under argon for $4 \mathrm{~h}$. The mixture was cooled and then washed with water. The organic layer was dried $\left(\mathrm{Na}_{2} \mathrm{CO}_{3}\right)$, filtered and concentrated. The resulting red solid was washed with hexanes to afford a red solid (110 mg, 92\%): ${ }^{1} \mathrm{H}$ NMR analysis was not successful due both to aggregation and the paramagnetic 
Co(II). LDMS obsd 891.1; FABMS obsd 891.2883, calcd $891.2874\left(\mathrm{C}_{54} \mathrm{H}_{49} \mathrm{CoN}_{4} \mathrm{O}_{3} \mathrm{P}\right) ; \lambda_{\text {abs }}=$ $413,529 \mathrm{~nm}$.

1-(4-Cyanophenyl)-1,1,1-tri-p-tolylmethane (20). A mixture of 19 (21.0 g, 47.6 $\mathrm{mmol})$ and $\mathrm{CuCN}(6.39 \mathrm{~g}, 71.4 \mathrm{mmol})$ in DMF $(125 \mathrm{~mL})$ was heated to reflux under argon for 18 $\mathrm{h}$. The reaction mixture was cooled and poured into $500 \mathrm{~mL}$ of aqueous ammonia. Air was bubbled through the mixture for $2 \mathrm{~h}$. The mixture was filtered and the resulting solid was washed with water until the washings were neutral $(\sim 1 \mathrm{~L})$. The solid was dissolved in toluene and the excess water was removed in a separatory funnel. The organic layer was dried $\left(\mathrm{Na}_{2} \mathrm{SO}_{4}\right)$, filtered, and concentrated. Column chromatography (silica, toluene, $7 \times 25 \mathrm{~cm}$ ) afforded a beige solid (11.1 g, 60.0\%): mp 207-209 ${ }^{\circ} \mathrm{C} ;{ }^{1} \mathrm{H}$ NMR $\delta 2.32$ (s, 9H), 7.03-7.08 (m, 12H), 7.36 (d, $J=$ $8.4 \mathrm{~Hz}, 2 \mathrm{H}), 7.52(\mathrm{~d}, J=8.4 \mathrm{~Hz}, 2 \mathrm{H}) ;{ }^{13} \mathrm{C}$ NMR $\delta 20.9,64.2,109.5,119.0,128.1,128.2,128.4$, 128.7, 130.4, 130.7, 130.90, 130.94, 131.2, 131.6, 135.8, 143.0, 152.9; FABMS obsd 388.2060, calcd 388.2065 (M + H); Anal. Calcd for $\mathrm{C}_{29} \mathrm{H}_{25} \mathrm{~N}$ : C, 89.89; H, 6.50; N, 3.61; Found: C, 89.21; $\mathrm{H}, 6.60 ; \mathrm{N}, 3.47$.

1,1,1-Tris[4-(diethoxyphosphorylmethyl)phenyl]-1-(4-formylphenyl)methane (24). A solution of $20(10.36 \mathrm{~g}, 26.7 \mathrm{mmol})$ in benzene $(200 \mathrm{~mL})$ was heated to reflux under argon. Then, samples of NBS (16.2 g, $90.9 \mathrm{mmol}, 3.3 \mathrm{eq})$ and AIBN (149 mg, $909 \mu \mathrm{mol})$ were added all at once. The mixture was refluxed for $20 \mathrm{~min}$ then cooled. The mixture was filtered through a silica pad $(7 \times 10 \mathrm{~cm}$, eluted with toluene) to remove succinimide. Removal of solvent afforded crude 1,1,1-tris[4-(bromomethyl)phenyl]-1-(4-cyanophenyl)methane (21) as an offwhite solid (16.5 g). Analysis by ${ }^{1} \mathrm{H}$ NMR spectroscopy showed $\sim 10 \%$ of tolyl resonances, indicating incomplete bromination. A solution of crude $21(6.00 \mathrm{~g}, 9.61 \mathrm{mmol})$ in $\mathrm{CH}_{2} \mathrm{Cl}_{2} /$ toluene $[60 \mathrm{~mL},(1: 1)]$ at $0{ }^{\circ} \mathrm{C}$ was treated dropwise with a solution of DIBALH (12.0 $\mathrm{mL}, 12.0 \mathrm{mmol}, 1.0 \mathrm{M}$ solution in hexanes) under argon for $1 \mathrm{~h}$. Then $\mathrm{CHCl}_{3}(80 \mathrm{~mL})$ and $2 \mathrm{~N}$ $\mathrm{HCl}(200 \mathrm{~mL})$ were added and the mixture was stirred at room temperature for $1 \mathrm{~h}$. The organic layer was separated, washed with water, dried $\left(\mathrm{Na}_{2} \mathrm{SO}_{4}\right)$, filtered and concentrated. Column chromatography (silica, $\mathrm{CH}_{2} \mathrm{Cl}_{2}$ ) afforded crude 1,1,1-tris[4-(bromomethyl)phenyl]-1-(4formylphenyl)methane (22) as a white solid $(4.93 \mathrm{~g})$. A solution of crude $\mathbf{2 2}(4.79 \mathrm{~g}, 7.64$ mmol) in $\mathrm{CH}_{2} \mathrm{Cl}_{2} /$ methanol [ $80 \mathrm{~mL},(1: 1)$ ] was treated with a solution of $\mathrm{TiCl}_{4}(153 \mu \mathrm{L}, 153$ $\mu \mathrm{mol}, 1.0 \mathrm{M}$ in $\left.\mathrm{CH}_{2} \mathrm{Cl}_{2}\right)$ at room temperature under argon for $30 \mathrm{~min}$. TEA $(153 \mu \mathrm{L})$ was added. After $30 \mathrm{~min}$, water $(50 \mathrm{~mL})$ was added. The organic phase was extracted with ethyl ether $(2 \mathrm{x}$ $100 \mathrm{~mL})$. The organic layer was dried $\left(\mathrm{Na}_{2} \mathrm{SO}_{4}\right)$, filtered, and concentrated to give crude $1,1,1$ tris[4-(bromomethyl)phenyl]-1-[4-(1,1-dimethoxymethyl)phenyl]methane (23) as an off-white solid $(4.51 \mathrm{~g})$. A mixture of crude $23(4.51 \mathrm{~g}, 6.70 \mathrm{mmol})$ in triethylphosphite $(50 \mathrm{~mL})$ was stirred under argon at $100{ }^{\circ} \mathrm{C}$ for $6 \mathrm{~h}$. The solution was cooled and poured into $1 \mathrm{~N} \mathrm{HCl}(250$ $\mathrm{mL})$. The mixture was extracted with ethyl acetate $(150 \mathrm{~mL})$. The layers were separated and the aqueous layer was washed with ethyl acetate $(2 \times 50 \mathrm{~mL})$. The organic layers were combined, washed with $5 \% \mathrm{NaHCO}_{3}$ and water. The organic layer was dried $\left(\mathrm{Na}_{2} \mathrm{SO}_{4}\right)$, filtered, and concentrated. Column chromatography [silica, ethyl acetate/methanol $(9: 1) \rightarrow(8: 2)$ ] afforded the title compound as a pale yellow oil $(2.89 \mathrm{~g}, 54 \%):{ }^{1} \mathrm{H}$ NMR $\delta 1.21(\mathrm{t}, J=6.8 \mathrm{~Hz}, 18 \mathrm{H}), 3.11$ $(\mathrm{d}, J=22.4 \mathrm{~Hz}, 6 \mathrm{H}), 4.00(\mathrm{~m}, 12 \mathrm{H}), 7.10(\mathrm{~d}, J=8.4 \mathrm{~Hz}, 6 \mathrm{H}), 7.18(\mathrm{~d}, J=8.4 \mathrm{~Hz}, 6 \mathrm{H}), 7.36(\mathrm{~d}, J$ $=8.4 \mathrm{~Hz}, 2 \mathrm{H}), 7.73(\mathrm{~d}, J=8.4 \mathrm{~Hz}, 2 \mathrm{H}), 9.98(\mathrm{~s}, 1 \mathrm{H}) ;{ }^{13} \mathrm{C} \mathrm{NMR} \delta 16.2,16.3,32.5,33.8,62.0$, $62.1,125.7,128.9,129.0,129.1,129.2,129.6,129.7,130.8,130.9,131.0,131.1,131.4,134.1$, 144.4, 153.6, 191.8; ${ }^{31} \mathrm{P}$ NMR $\delta$ 27.0; FABMS obsd 799.2968, calcd 799.2930 $(\mathrm{M}+\mathrm{H})$ $\left(\mathrm{C}_{41} \mathrm{H}_{53} \mathrm{O}_{10} \mathrm{P}_{3}\right)$. 


\section{5-[4-[1,1,1-Tris[4-}

(diethoxyphosphorylmethyl)phenyl]methyl]phenyl]dipyrromethane (25). Following a standard procedure, ${ }^{39}$ a solution of $\mathbf{2 4}(2.83 \mathrm{~g}, 3.54 \mathrm{mmol})$ in pyrrole $(24.7 \mathrm{~mL}, 354 \mathrm{mmol})$ was degassed with argon for $10 \mathrm{~min}$. A sample of $\mathrm{InCl}_{3}(78 \mathrm{mg}, 0.354 \mathrm{mmol})$ was added and the mixture was stirred at room temperature for $90 \mathrm{~min}$. Powdered $\mathrm{NaOH}(425 \mathrm{mg})$ was added and the mixture was stirred for $30 \mathrm{~min}$. The mixture was filtered and the pyrrole was removed under vacuum. Column chromatography [silica, ethyl acetate/MeOH (3:1)] afforded a pale yellow foam $(2.50 \mathrm{~g}, 77 \%): \mathrm{mp} 58-61{ }^{\circ} \mathrm{C} ;{ }^{1} \mathrm{H} \mathrm{NMR} \delta 1.21(\mathrm{t}, J=6.8 \mathrm{~Hz}, 18 \mathrm{H}), 3.10(\mathrm{~d}, J=21.6 \mathrm{~Hz}$, $6 \mathrm{H}), 3.99(\mathrm{~m}, 12 \mathrm{H}), 5.44,(\mathrm{~s}, 1 \mathrm{H}), 5.89(\mathrm{br} \mathrm{s}, 2 \mathrm{H}), 6.13(\mathrm{~m}, 2 \mathrm{H}), 6.67(\mathrm{~m}, 2 \mathrm{H}), 7.06-7.17(\mathrm{~m}$, 16H), 8.10 (br s, 2H); ${ }^{13} \mathrm{C}$ NMR $\delta 16.1,16.2,32.3,33.7,43.2,62.0,62.1,63.7,106.9,107.9$, 117.1, 127.3, 128.77, 128.84, 128.9, 129.0, 130.8, 130.95, 130.98, 132.4, 140.0, 144.8, 145.1, 145.2; ${ }^{31} \mathrm{P}$ NMR $\delta$ 27.1; FABMS obsd 915.3679, calcd 915.3668 $(\mathrm{M}+\mathrm{H})$; Anal. Calcd for $\mathrm{C}_{49} \mathrm{H}_{61} \mathrm{~N}_{2} \mathrm{O}_{9} \mathrm{P}_{3}$ : C, 64.32; H, 6.72; O, 15.74; Found: C, 63.99; H, 6.64; O, 15.55.

\section{Zn(II)-5-[4-[1,1,1-Tris[4-(diethoxyphosphorylmethyl)phenyl]methyl]phenyl]-}

10,15,20-tri-p-tolylporphyrin (Zn26). Following a standard procedure, ${ }^{32}$ a solution of 10 (120 $\mathrm{mg}, 250 \mu \mathrm{mol})$ in dry THF/methanol [11 mL, (10:1)] was treated with $\mathrm{NaBH}_{4}(190 \mathrm{mg}, 5.10$ $\mathrm{mmol}$ ). After $40 \mathrm{~min}$, the reaction mixture was poured into a stirred mixture of saturated aqueous $\mathrm{NH}_{4} \mathrm{Cl}(150 \mathrm{~mL})$ and $\mathrm{CH}_{2} \mathrm{Cl}_{2}(50 \mathrm{~mL})$. The organic phase was washed with water and brine, dried $\left(\mathrm{Na}_{2} \mathrm{SO}_{4}\right)$, and concentrated. The resulting 10-diol was dissolved in $\mathrm{CH}_{2} \mathrm{Cl}_{2}(100$ $\mathrm{mL})$ and treated with $25(225 \mathrm{mg}, 250 \mu \mathrm{mol})$ and $\mathrm{InCl}_{3}(7.0 \mathrm{mg}, 33 \mu \mathrm{mol})$ under argon for $1 \mathrm{~h}$ at room temperature. DDQ $(170 \mathrm{mg}, 750 \mu \mathrm{mol})$ was added. After $1 \mathrm{~h}$, TEA (400 $\mu \mathrm{L})$ was added. Column chromatography [silica, ethyl acetate/methanol (9:1)] afforded a purple solid (60 mg). The solid $(60 \mathrm{mg}, 0.045 \mathrm{mmol})$ was dissolved in $\mathrm{CHCl}_{3}(30 \mathrm{~mL})$ and treated overnight with a solution of $\mathrm{Zn}(\mathrm{OAc})_{2} \cdot 2 \mathrm{H}_{2} \mathrm{O}(60 \mathrm{mg}, 0.27 \mathrm{mmol})$ in methanol $(5 \mathrm{~mL})$. Column chromatography [silica, ethyl acetate/methanol, (9:1)] afforded a purple solid (40 mg, 63\%): ${ }^{1} \mathrm{H}$ NMR $\left(\mathrm{THF}-\mathrm{d}_{8}\right) \delta$ 1.15-1.18 (m, 18H), 2.68-2.69 (m, 9H), $3.10(\mathrm{~s}, 3 \mathrm{H}), 3.16(\mathrm{~s}, 3 \mathrm{H}), 3.90-3.94(\mathrm{~m}, 12 \mathrm{H}), 7.32-$ $7.34(\mathrm{~m}, 6 \mathrm{H}), 7.41-7.43(\mathrm{~m}, 6 \mathrm{H}), 7.54-7.59(\mathrm{~m}, 8 \mathrm{H}), 8.05-8.09(\mathrm{~m}, 8 \mathrm{H}), 8.83-8.89(\mathrm{~m}, 8 \mathrm{H}) ;{ }^{31} \mathrm{P}$ NMR $\delta$ 26.71; LDMS obsd 1413.85; FABMS obsd 1410.4476, calcd 1410.4508; $\left(\mathrm{C}_{81} \mathrm{H}_{81} \mathrm{~N}_{4} \mathrm{O}_{9} \mathrm{P}_{3} \mathrm{Zn}\right) ; \lambda_{\text {abs }}=427,558,598 \mathrm{~nm}$.

\section{Zn(II)-5-[4-[1,1,1-Tris[4-(dihydroxyphosphorylmethyl)phenyl]methyl]phenyl]-}

10,15,20-tri-p-tolylporphyrin (Zn27). A solution of $\mathbf{Z n 2 6}(33 \mathrm{mg}, 23.4 \mu \mathrm{mol})$ in $\mathrm{CHCl}_{3}(2.3$ $\mathrm{mL})$ was treated with TEA $(65 \mu \mathrm{L}, 470 \mu \mathrm{mol})$ and $\mathrm{TMS}-\mathrm{Br}(46 \mu \mathrm{L}, 350 \mu \mathrm{mol})$ and the mixture was refluxed under argon. After $2 \mathrm{~h}$, the solution was cooled and $\mathrm{CHCl}_{3}(10 \mathrm{~mL})$ and water $(10$ $\mathrm{mL}$ ) were added. The porphyrin preferentially dissolved in the aqueous layer. The mixture was concentrated. The solid was suspended in hexanes, sonicated for $10 \mathrm{~min}$, and centrifuged. The supernatant was decanted. This process was repeated once. The solid was dissolved in methanol with sonication. The solution was poured into diethyl ether and the precipitate was collected to afford a purple solid (24 mg, 82\%): FABMS obsd 1242.2656, calcd 1242.2630 $\left(\mathrm{C}_{69} \mathrm{H}_{57} \mathrm{~N}_{4} \mathrm{O}_{9} \mathrm{P}_{3} \mathrm{Zn}\right) ; \lambda_{\mathrm{abs}}=423,522,558,599 \mathrm{~nm}$.

5-(4-Ethynylphenyl)-1,9-bis(4-methylbenzoyl)dipyrromethane (29). A solution of 28 $(2.00 \mathrm{~g}, 3.61 \mathrm{mmol})$ in $\mathrm{CHCl}_{3}(75 \mathrm{~mL})$ was treated with TBAF $(4.33 \mathrm{~mL}, 4.33 \mathrm{mmol}, 1.0 \mathrm{M}$ in $\mathrm{THF}$ ) at room temperature under argon for $1 \mathrm{~h}$. The solution was then washed with $10 \%$ aqueous $\mathrm{NaHCO}_{3}$ and water. The organic phase was dried $\left(\mathrm{Na}_{2} \mathrm{SO}_{4}\right)$, filtered and concentrated. Column chromatography [silica, $\mathrm{CH}_{2} \mathrm{Cl}_{2}$ /ethyl acetate (95:5)] afforded an orange foam $(1.37 \mathrm{~g}, 79 \%)$ : $\mathrm{mp}$ 122-126 ${ }^{\circ} \mathrm{C} ;{ }^{1} \mathrm{H}$ NMR $\delta 2.39(\mathrm{~s}, 6 \mathrm{H}), 3.10(\mathrm{~s}, 1 \mathrm{H}), 5.67(\mathrm{~s}, 1 \mathrm{H}), 5.92(\mathrm{~m}, 2 \mathrm{H}), 6.51(\mathrm{~m}, 2 \mathrm{H}), 7.19$ 
$(\mathrm{d}, J=8.0 \mathrm{~Hz}, 4 \mathrm{H}), 7.53(\mathrm{~s}, 4 \mathrm{H}), 7.66(\mathrm{~d}, J=8.0 \mathrm{~Hz}, 4 \mathrm{H}), 11.64(\mathrm{br} \mathrm{s}, 2 \mathrm{H}) ;{ }^{13} \mathrm{C}$ NMR $\delta 21.5$, 44.9, 77.3, 83.5, 111.2, 120.6, 121.0, 128.6, 128.9, 129.8, 131.2, 132.5, 135.4, 140.3, 141.4, 142.2, 184.3; FABMS obsd 483.2059, calcd 483.2073 [M+ H]; Anal. Calcd for $\mathrm{C}_{33} \mathrm{H}_{26} \mathrm{~N}_{2} \mathrm{O}_{2}$ : C, 82.13; H, 5.43; Found: C, 81.54; H, 5.54.

Zn(II)-5-[4-[1,1,1-Tris[4-(diethoxyphosphorylmethyl)phenyl]methyl]phenyl-15-(4ethynylphenyl)-10,20-di-p-tolylporphyrin (Zn30). Following a standard procedure, ${ }^{32}$ a solution of $29(500 \mathrm{mg}, 1.04 \mathrm{mmol})$ in $\mathrm{THF} / \mathrm{MeOH}$ [38 mL, (10:1)] was treated with $\mathrm{NaBH}_{4}$ (784 mg, $20.7 \mathrm{mmol}$ ). After $40 \mathrm{~min}$, the reaction mixture was poured into a mixture of saturated aqueous $\mathrm{NH}_{4} \mathrm{Cl}(100 \mathrm{~mL})$ and $\mathrm{CH}_{2} \mathrm{Cl}_{2}(100 \mathrm{~mL})$. The organic phase was collected, dried $\left(\mathrm{Na}_{2} \mathrm{SO}_{4}\right)$, and concentrated to a yellow foam. The resulting 29-diol was dissolved in $\mathrm{CH}_{2} \mathrm{Cl}_{2}$ $(414 \mathrm{~mL})$ and samples of 25 (950 mg, $1.04 \mathrm{mmol})$ and $\mathrm{Yb}(\mathrm{OTf})_{3}(822 \mathrm{mg}, 1.33 \mathrm{mmol})$ were added. After $25 \mathrm{~min}$, DDQ (705 mg, $3.12 \mathrm{mmol}$ ) was added followed by TEA ( $1 \mathrm{~mL})$. After 30 min, the reaction mixture was chromatographed [silica, ethyl acetate/MeOH (8:2)] to recover the partially purified free base porphyrin. The solid was dissolved in $\mathrm{CHCl}_{3}(60 \mathrm{~mL})$ and treated with a solution of $\mathrm{Zn}(\mathrm{OAc})_{2} \cdot 2 \mathrm{H}_{2} \mathrm{O}(228 \mathrm{mg}, 1.04 \mathrm{mmol})$ in methanol $(10 \mathrm{~mL})$ for $12 \mathrm{~h}$. Column chromatography [silica, ethyl acetate/MeOH $(9: 1) \rightarrow(8: 2)$; silica, $\mathrm{CHCl}_{3} / \mathrm{MeOH}$ (5:1)] afforded a purple solid $(335 \mathrm{mg}, 24 \%)$ : ${ }^{1} \mathrm{H}$ NMR $\delta 1.16(\mathrm{t}, J=7.2 \mathrm{~Hz}, 18 \mathrm{H}), 2.69(\mathrm{~s}, 6 \mathrm{H}), 3.12(\mathrm{~d}, J=$ $21.6 \mathrm{~Hz}, 6 \mathrm{H}), 3.77(\mathrm{~s}, 1 \mathrm{H}), 3.90(\mathrm{~m}, 12 \mathrm{H}), 7.30-7.33(\mathrm{~m}, 6 \mathrm{H}), 7.41(\mathrm{~d}, J=8.0 \mathrm{~Hz}, 6 \mathrm{H}), 7.55-$ $7.59(\mathrm{~m}, 6 \mathrm{H}), 7.85(\mathrm{~d}, J=8.0 \mathrm{~Hz}, 2 \mathrm{H}), 8.06-8.10(\mathrm{~m}, 6 \mathrm{H}), 8.17(\mathrm{~d}, J=8.0 \mathrm{~Hz}, 2 \mathrm{H}), 8.81(\mathrm{~d}, J=$ $4.4 \mathrm{~Hz}, 2 \mathrm{H}), 8.86-8.90(\mathrm{~m}, 6 \mathrm{H}) ;{ }^{31} \mathrm{P}$ NMR $\delta$ 26.6; MALDI-MS (POPOP) 1424.9; FABMS obsd 1420.4305, calcd 1420.4351 $\left(\mathrm{C}_{82} \mathrm{H}_{79} \mathrm{~N}_{4} \mathrm{O}_{9} \mathrm{P}_{3} \mathrm{Zn}\right) ; \lambda_{\text {abs }}=427,555,601 \mathrm{~nm}$.

Electrochemical Studies of Monolayers on $\mathrm{SiO}_{2}$. Redox-active molecules bearing phosphonic acid groups were tethered to thin layers of $\mathrm{SiO}_{2}$ grown on (100) p-type $\mathrm{Si}$ substrates with a doping density of $1 \times 10^{18} \mathrm{~cm}^{-3}$ (obtained from MEMC, Inc.). The sample was cleaned using a JTB-111 clean for 10 min. A thin $\mathrm{SiO}_{2}$ layer $(4.5 \mathrm{~nm})$ was grown via furnace thermal oxidation at $900{ }^{\circ} \mathrm{C}$ in $\mathrm{O}_{2}$. This layer was then further thinned by subjecting the oxide layer to a slow etch in $1 \% \mathrm{HF}$ solution for $\sim 35 \mathrm{sec}$, affording a $1.3 \mathrm{~nm}$ layer of $\mathrm{SiO}_{2}$ as determined by ellipsometry. A solution of the redox-active compound (Zn27) in DMF was added dropwise (8 drops) to the thin $\mathrm{SiO}_{2}$ surface. After each drop, the substrate was heated to $170{ }^{\circ} \mathrm{C}$ for $10 \mathrm{~min}$. The sample was then rinsed in DMF and $\mathrm{CH}_{2} \mathrm{Cl}_{2}$. The substrate was then placed on a probe station and the backside (working electrode) was contacted via the chuck. A solution of $1.0 \mathrm{M}$ $\mathrm{Bu}_{4} \mathrm{NPF}_{6}$ in propylene carbonate was placed on top of the molecules and used as the conducting gate electrolyte. A silver gate electrode was immersed in the electrolyte and used as the reference electrode. Traditional cyclic voltammetry measurements were obtained using a custom program run using National Instruments LabVIEW. 


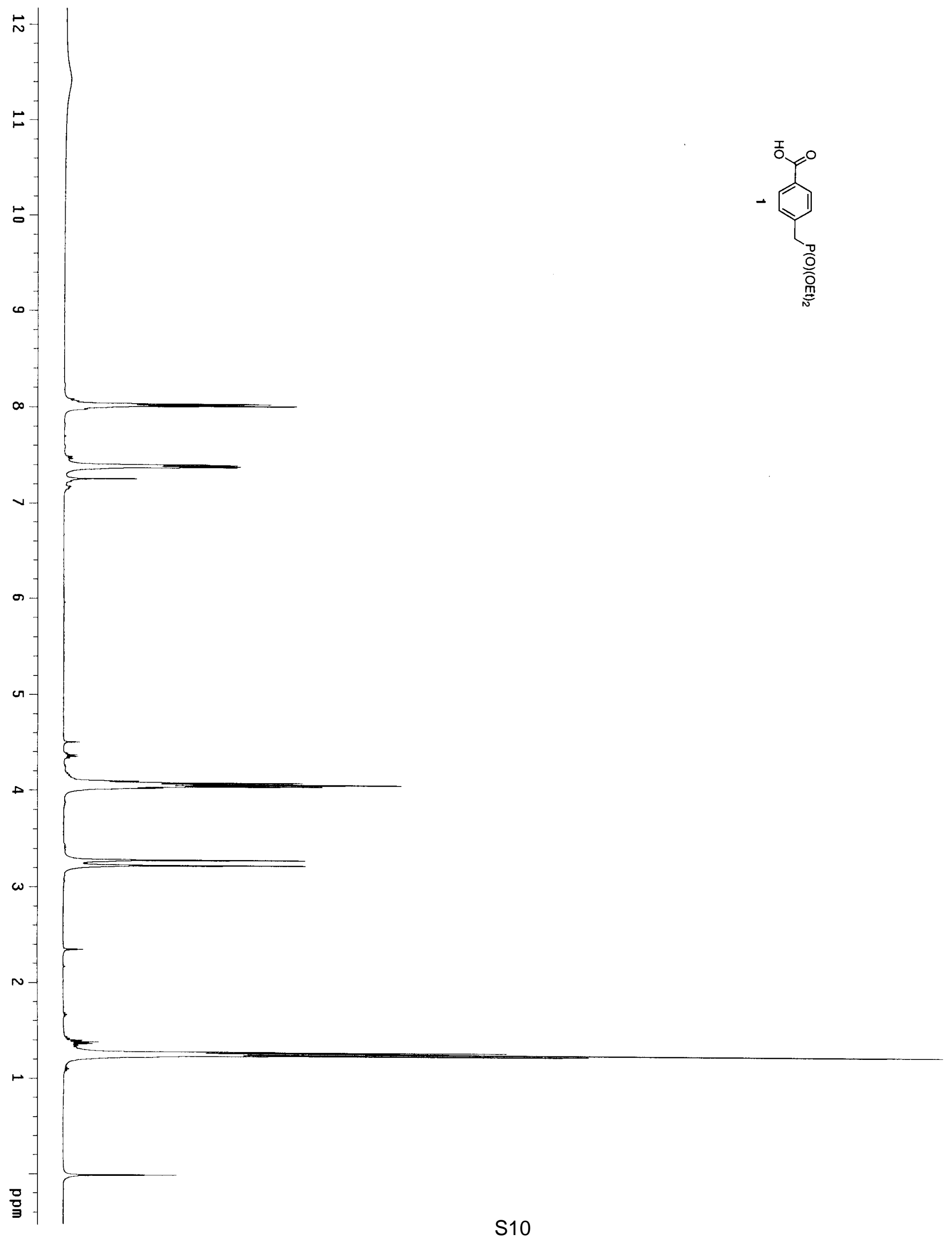




$$
\mid
$$




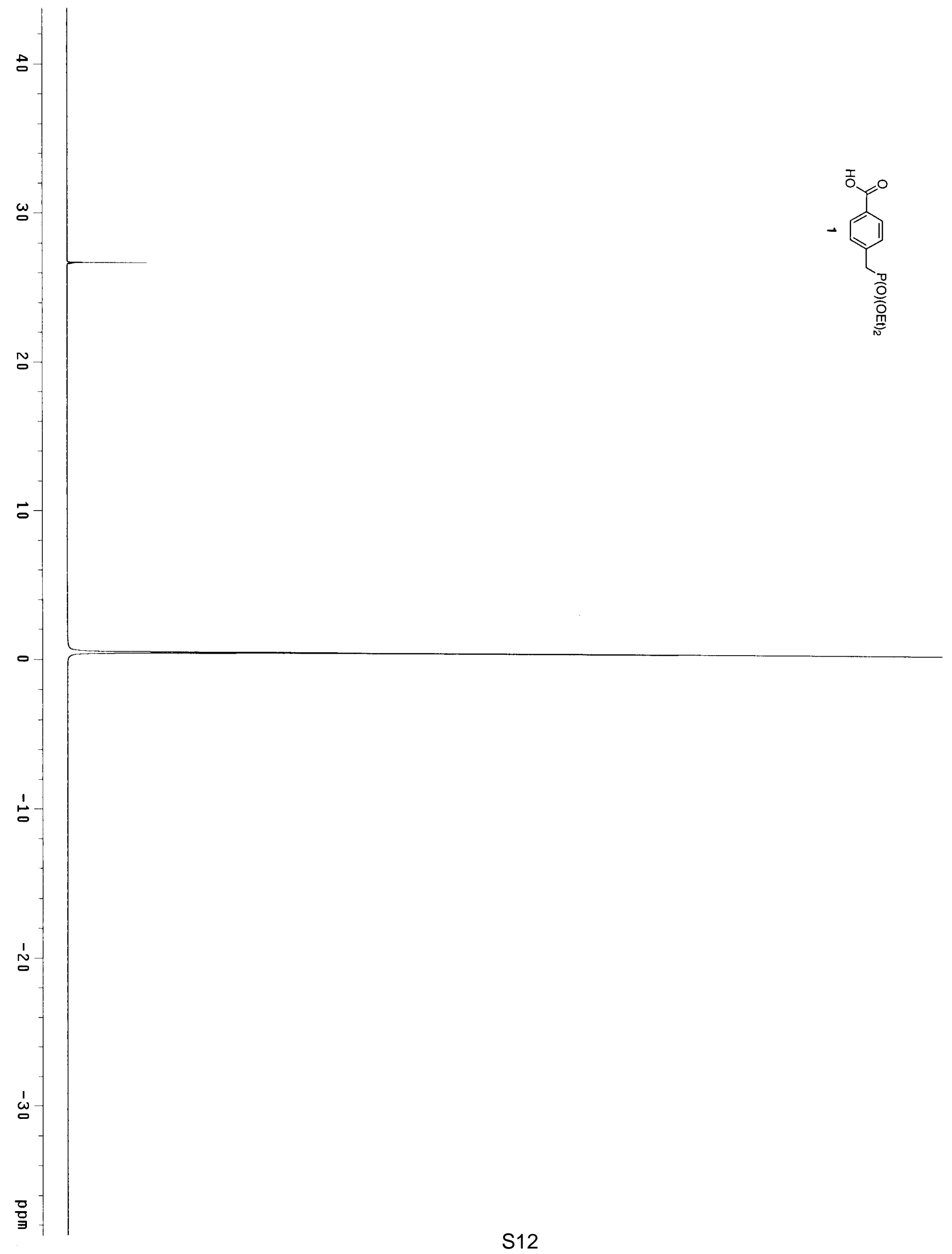





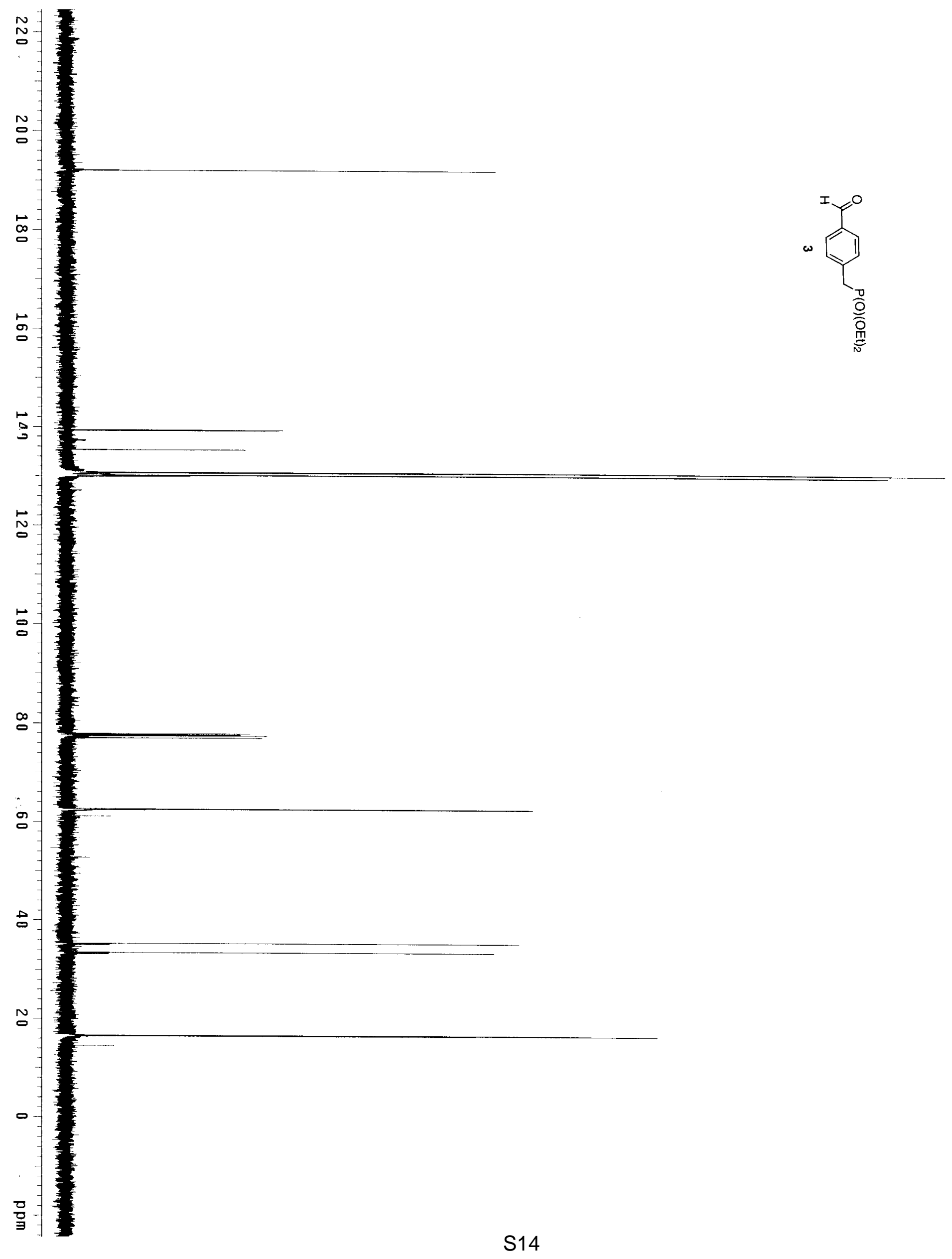




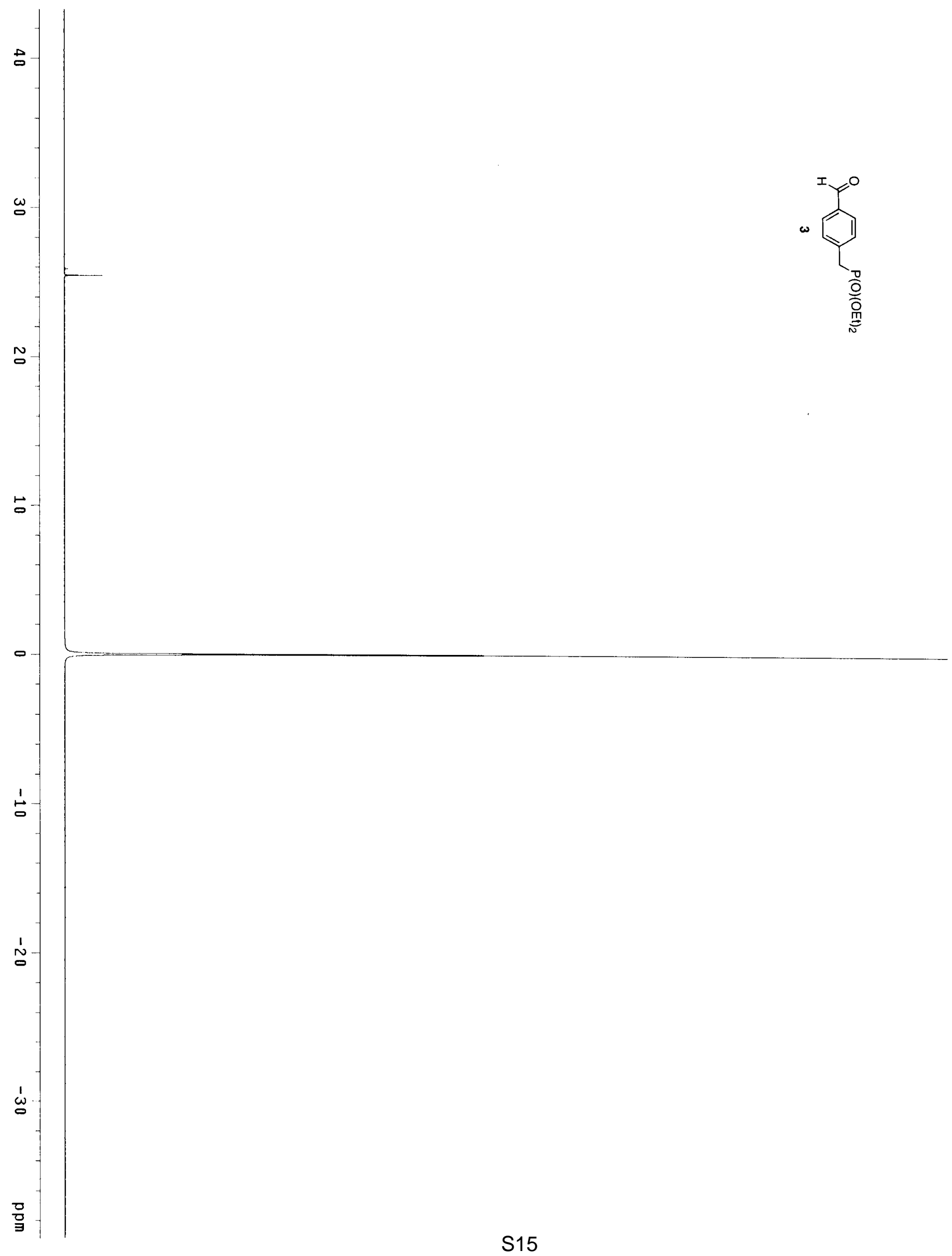




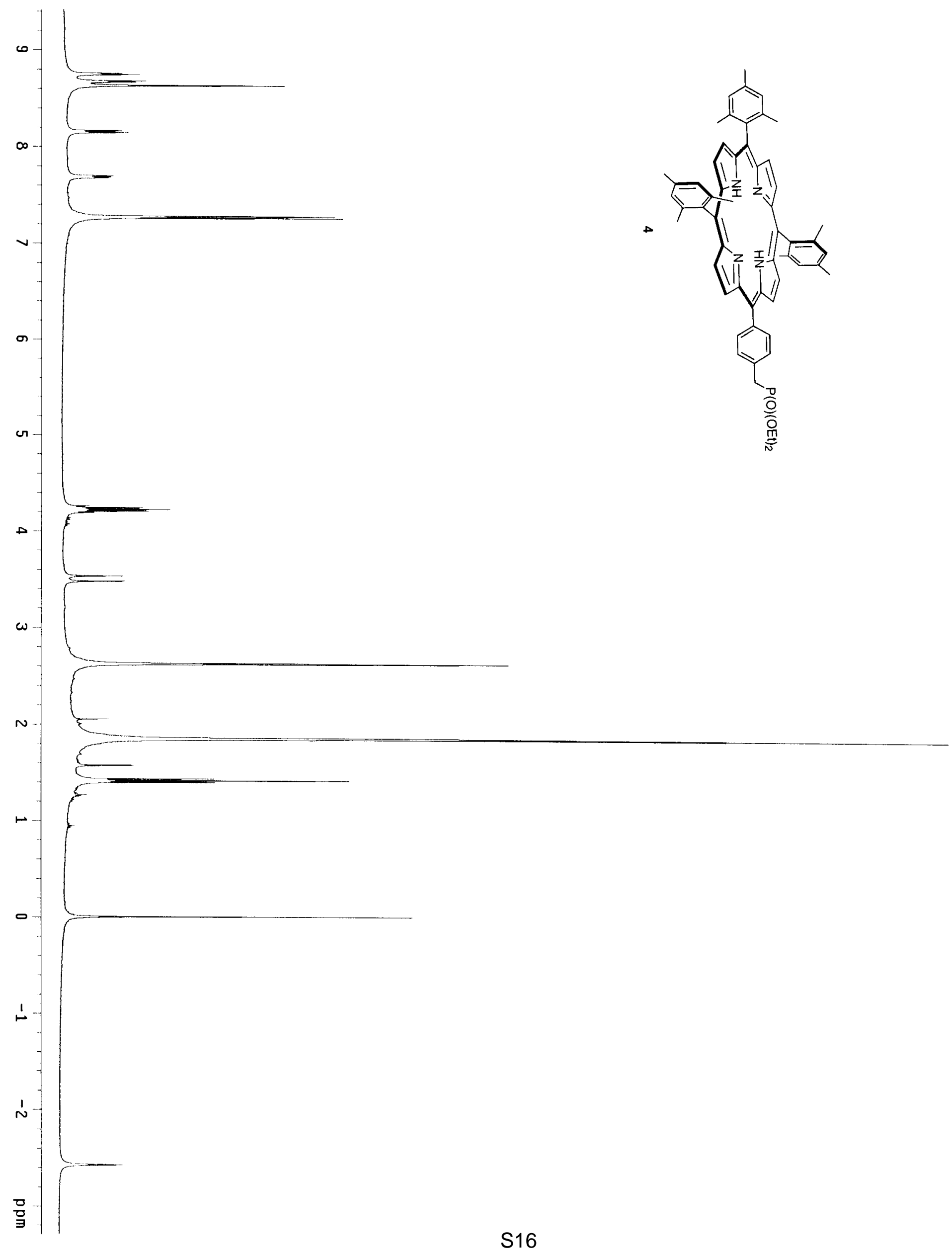




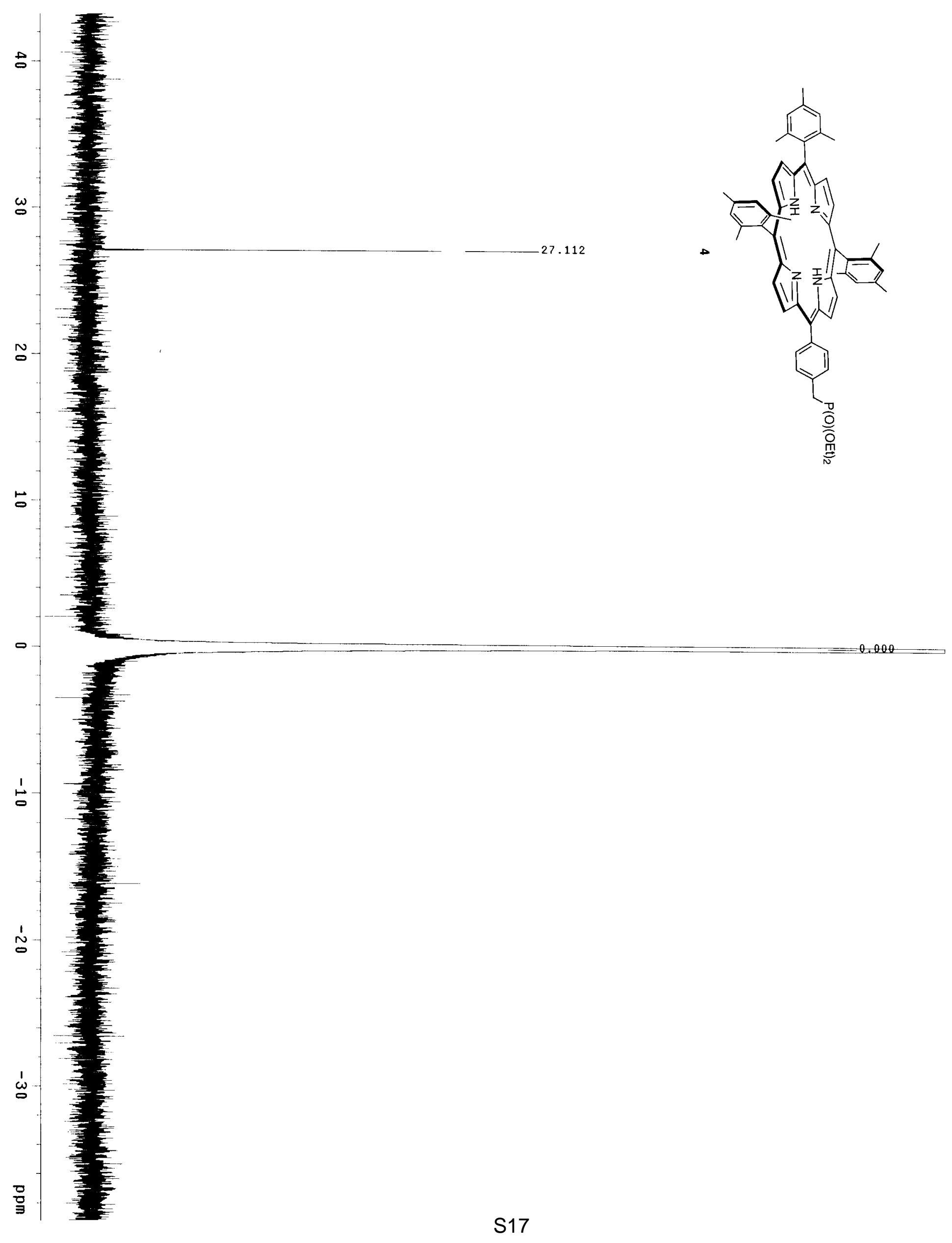




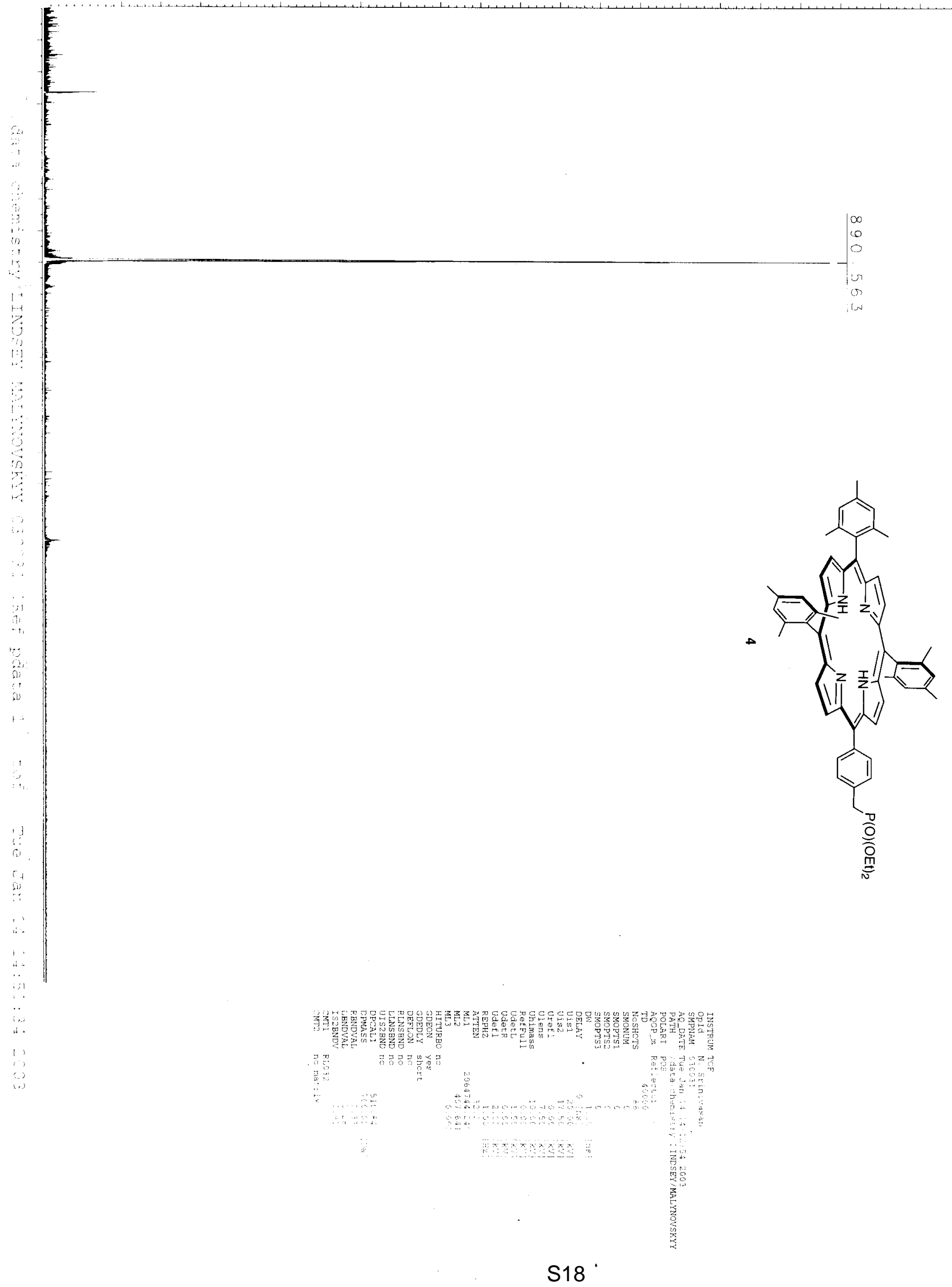




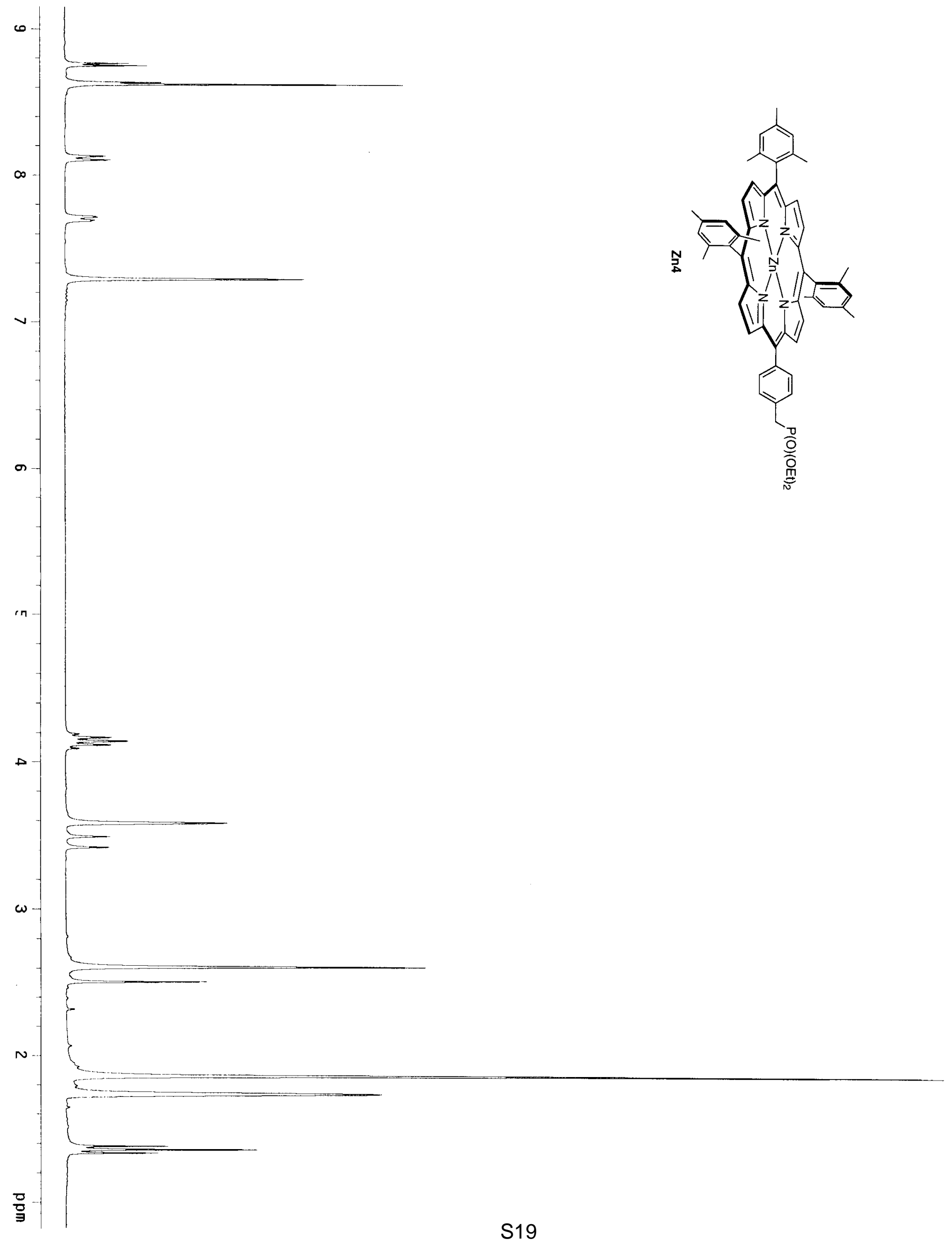




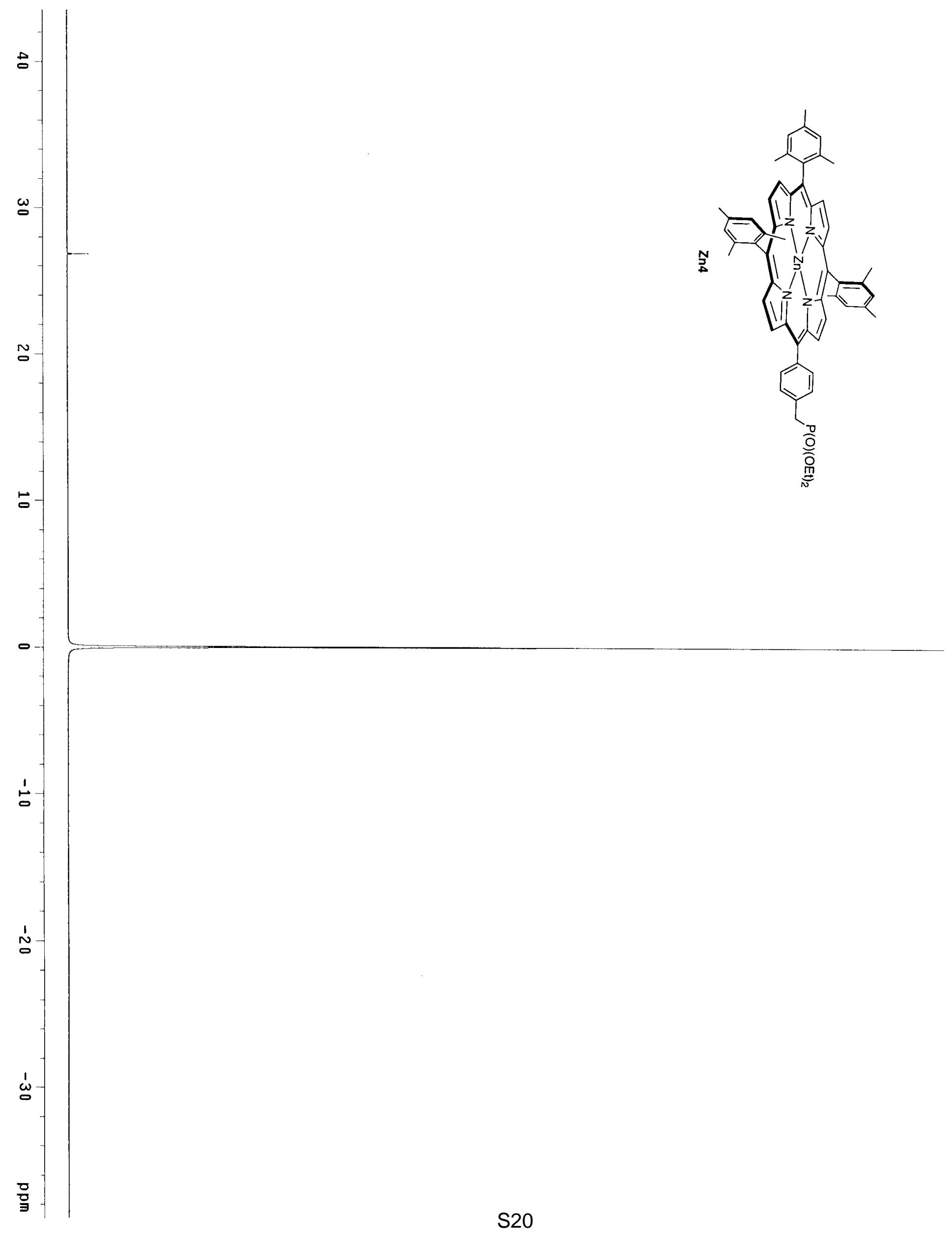




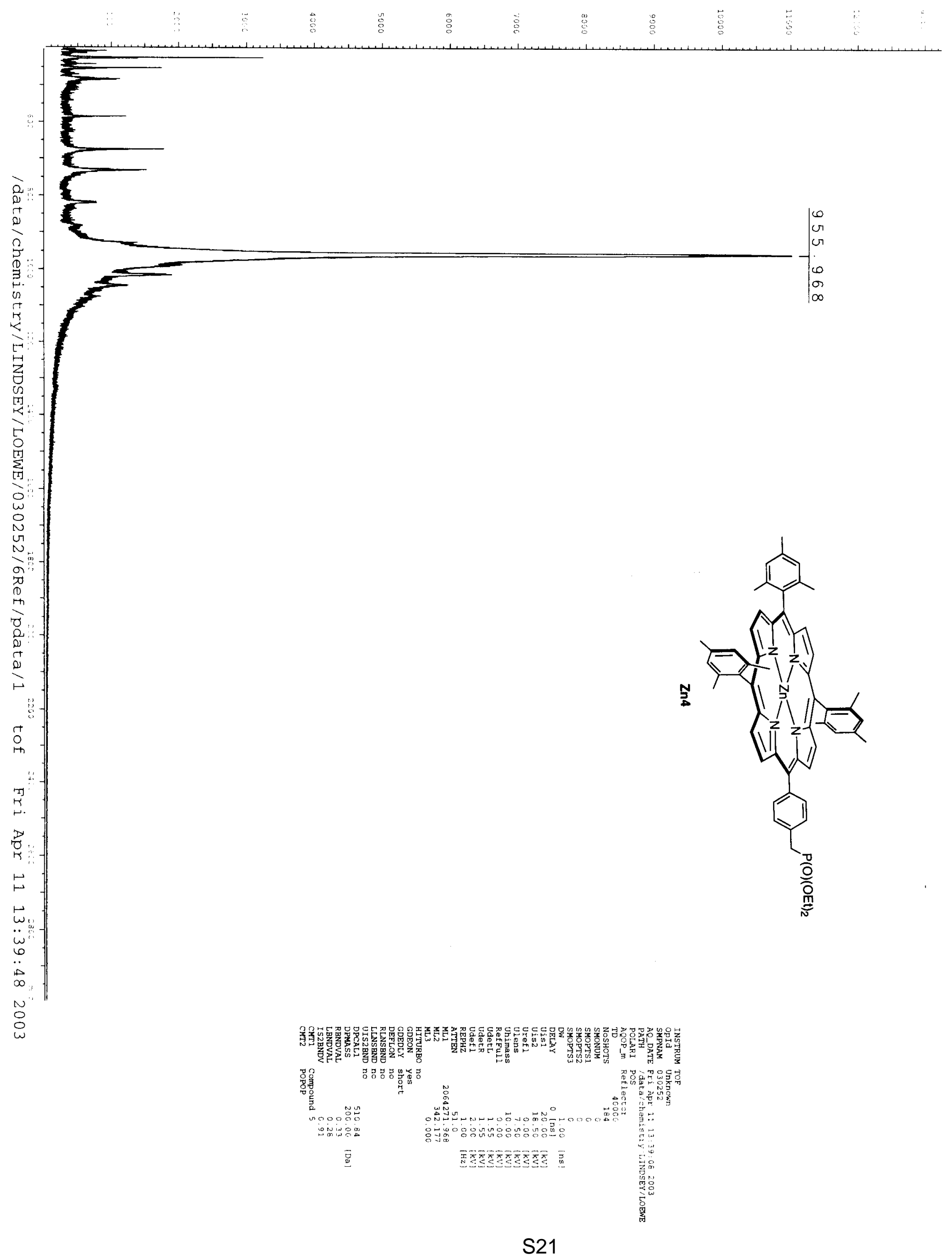




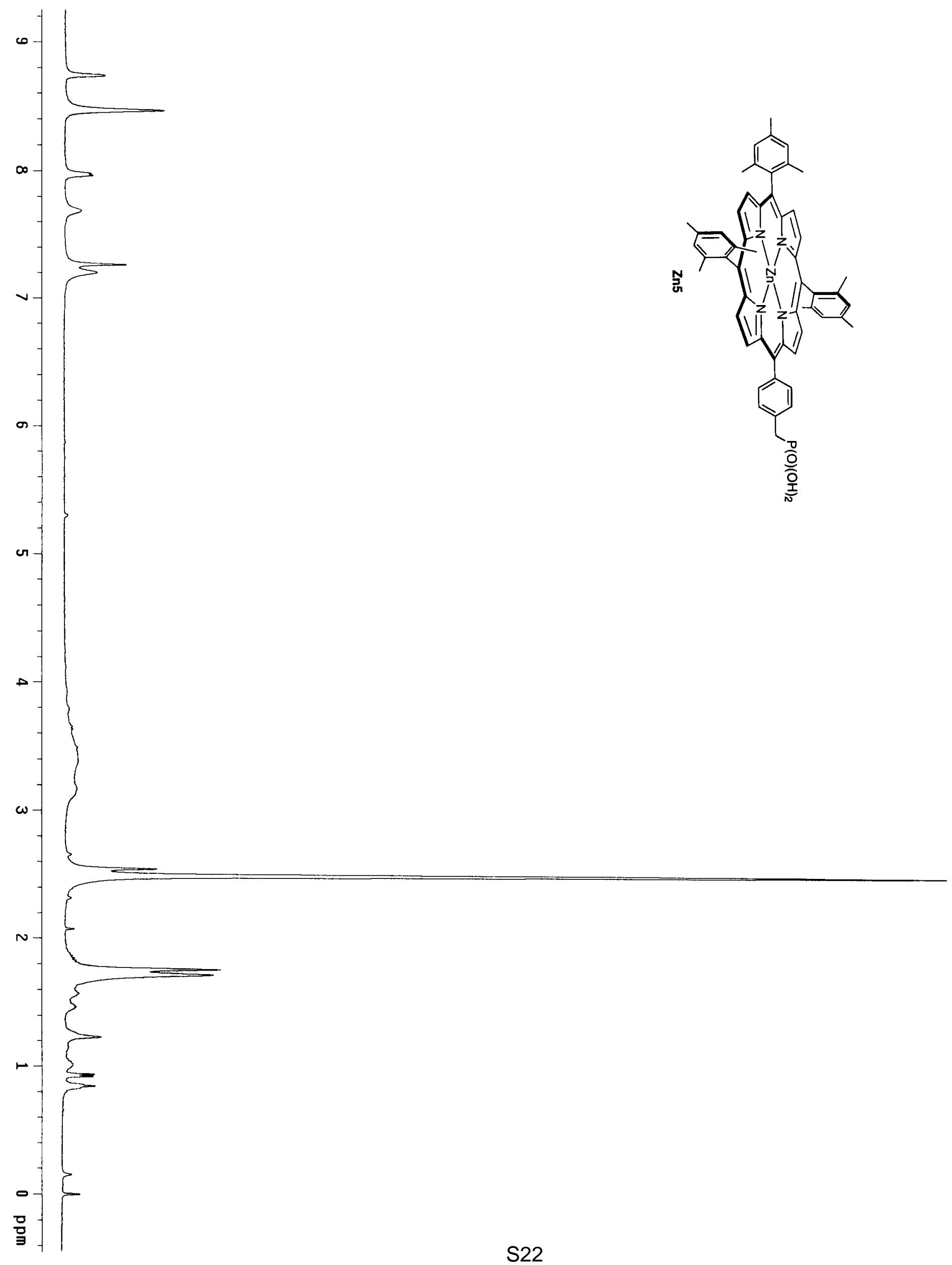



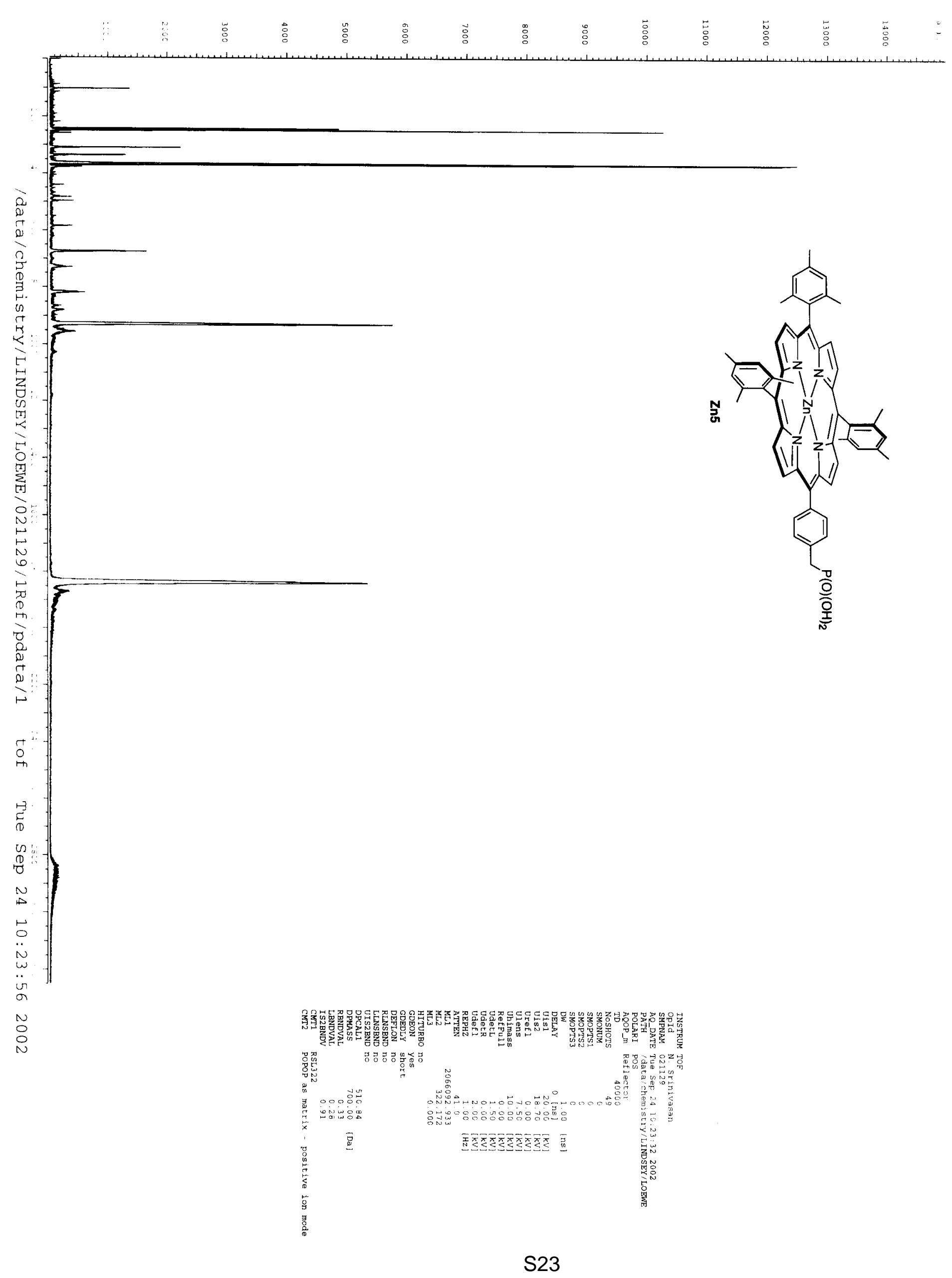


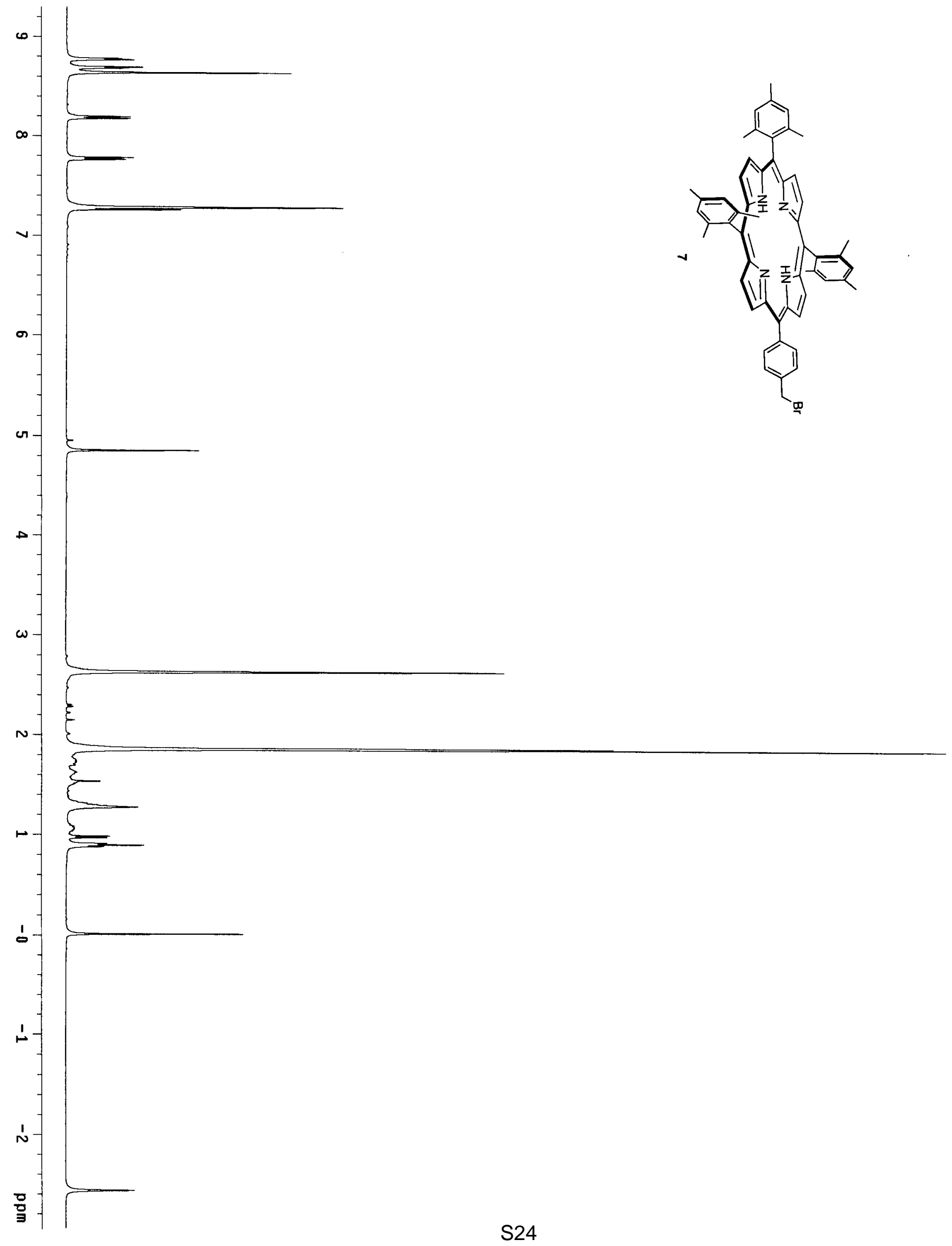



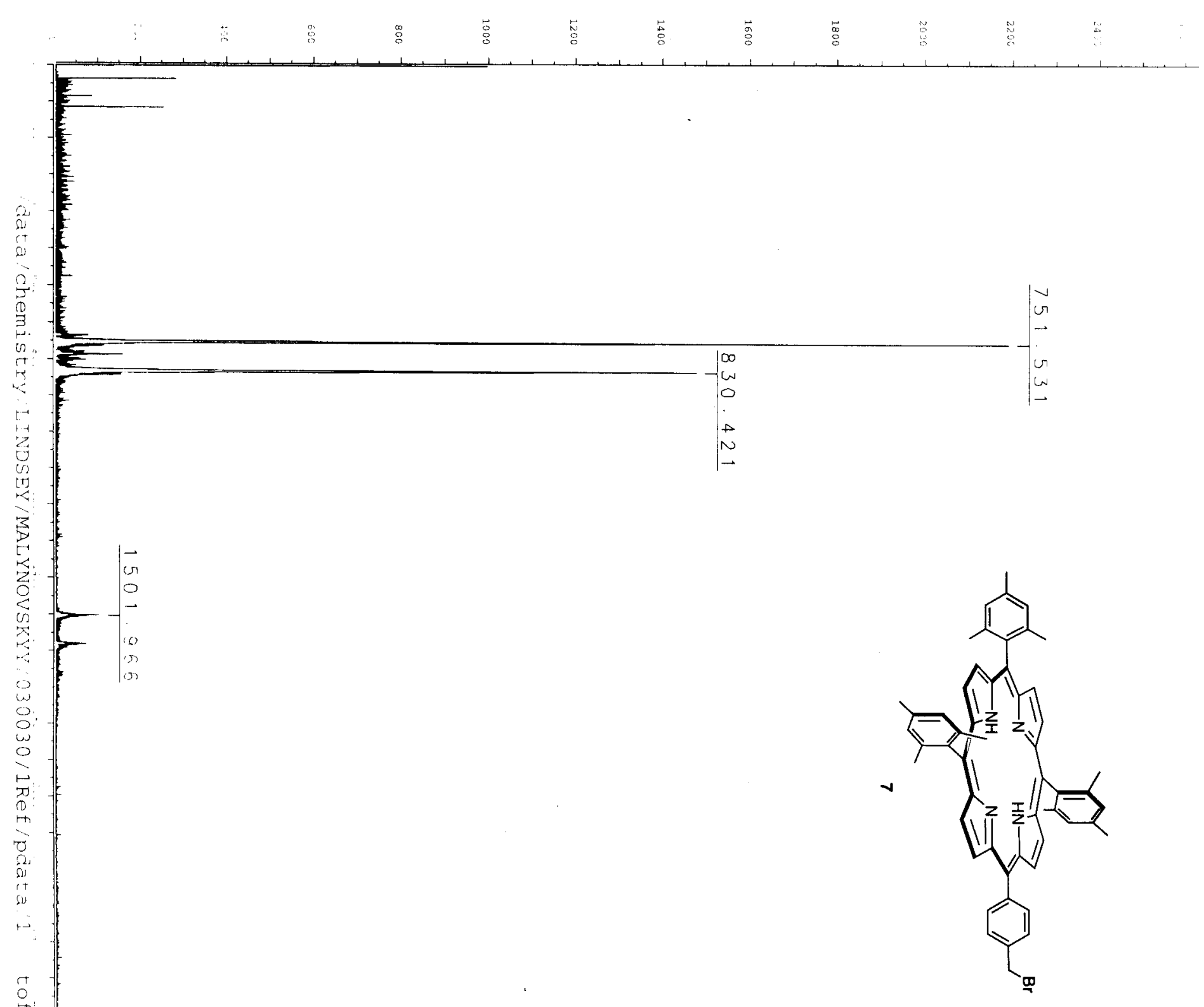


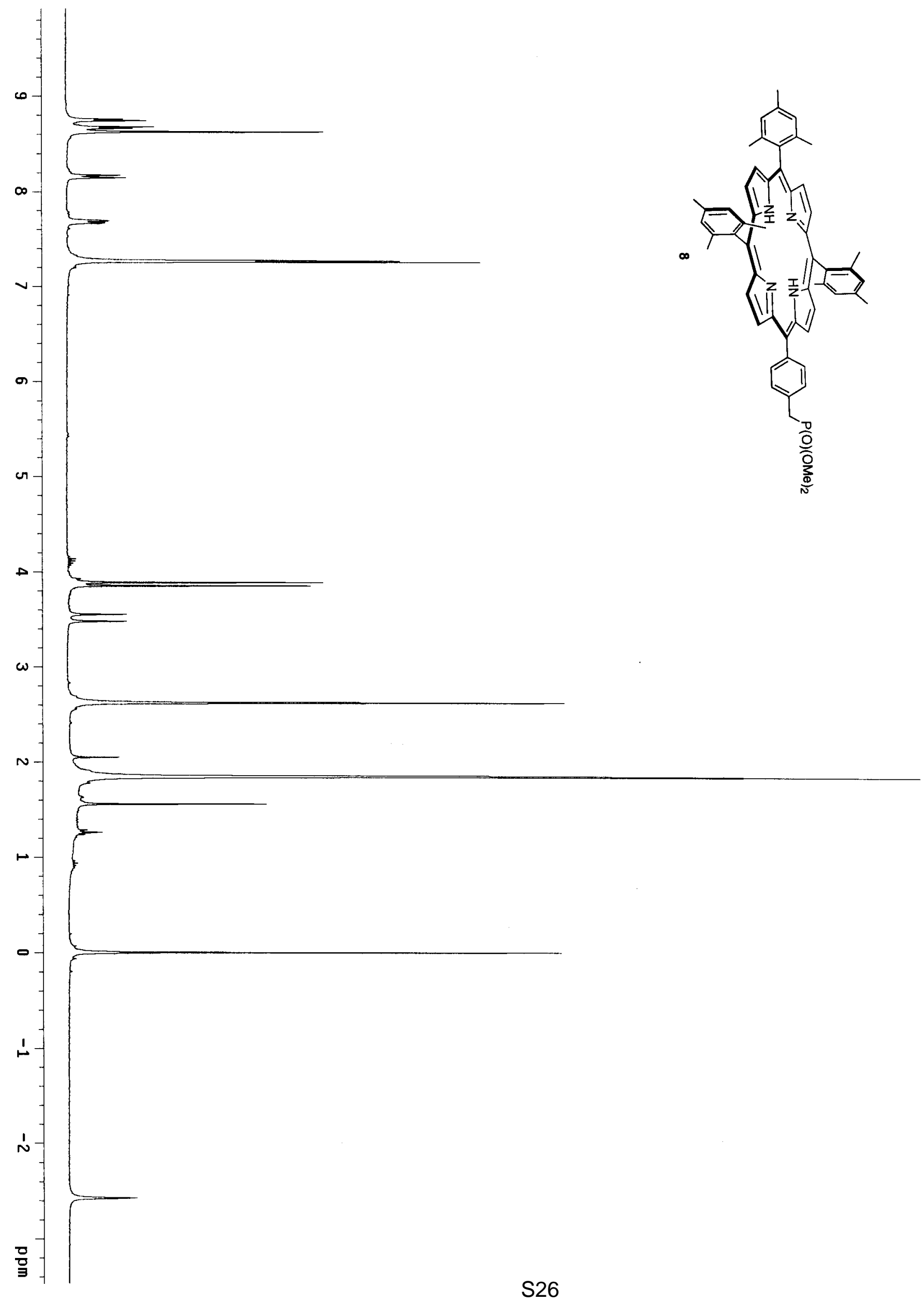




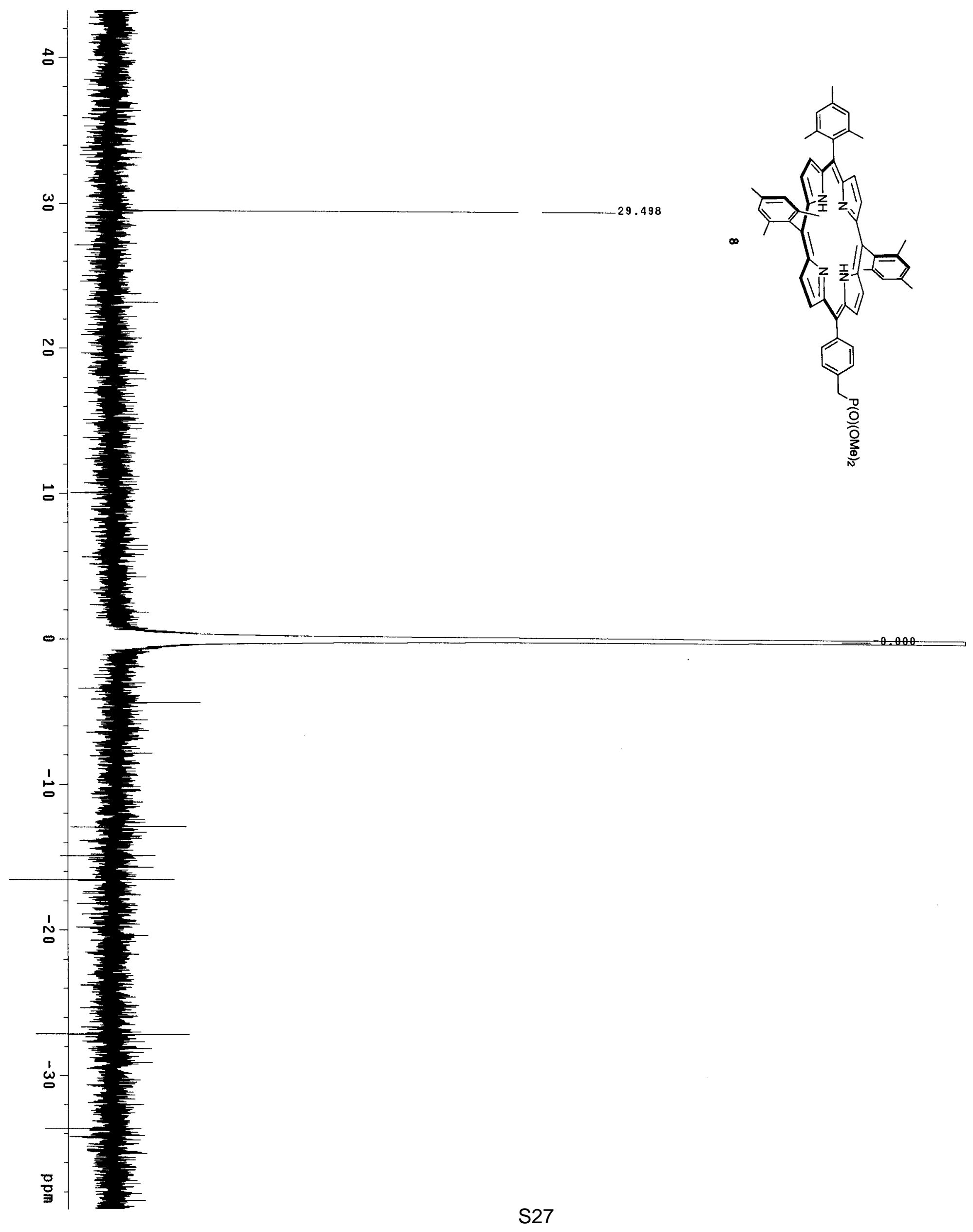




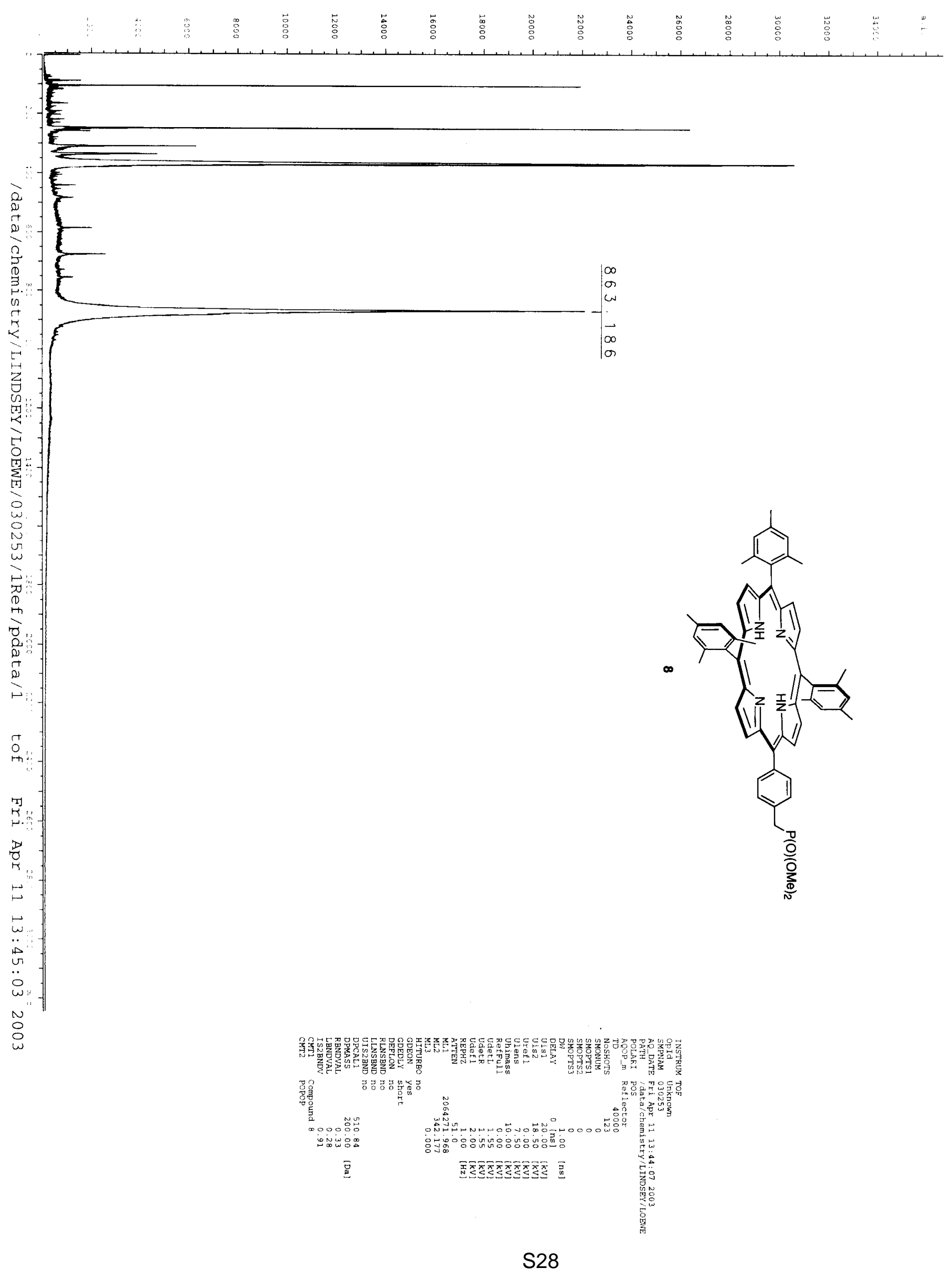




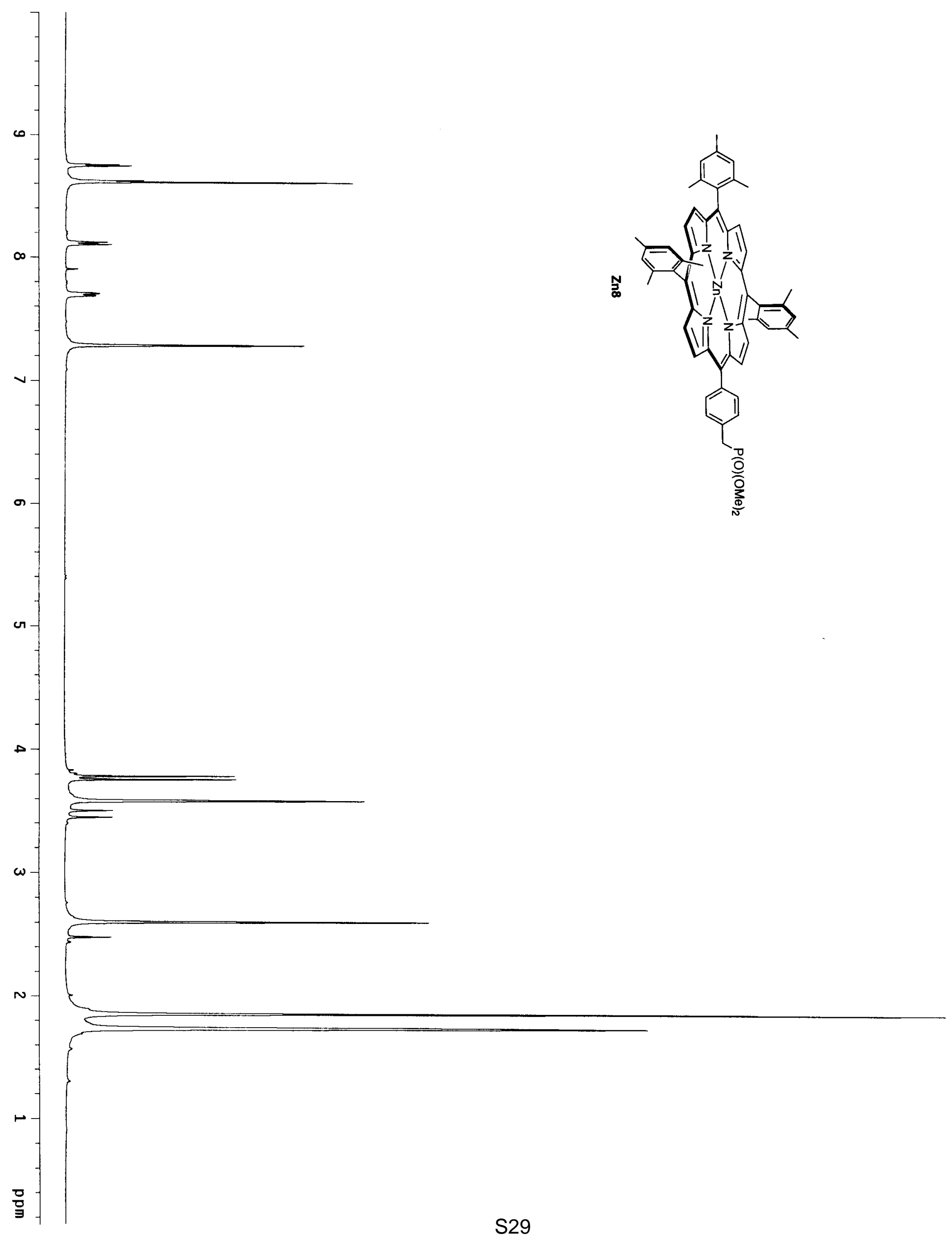




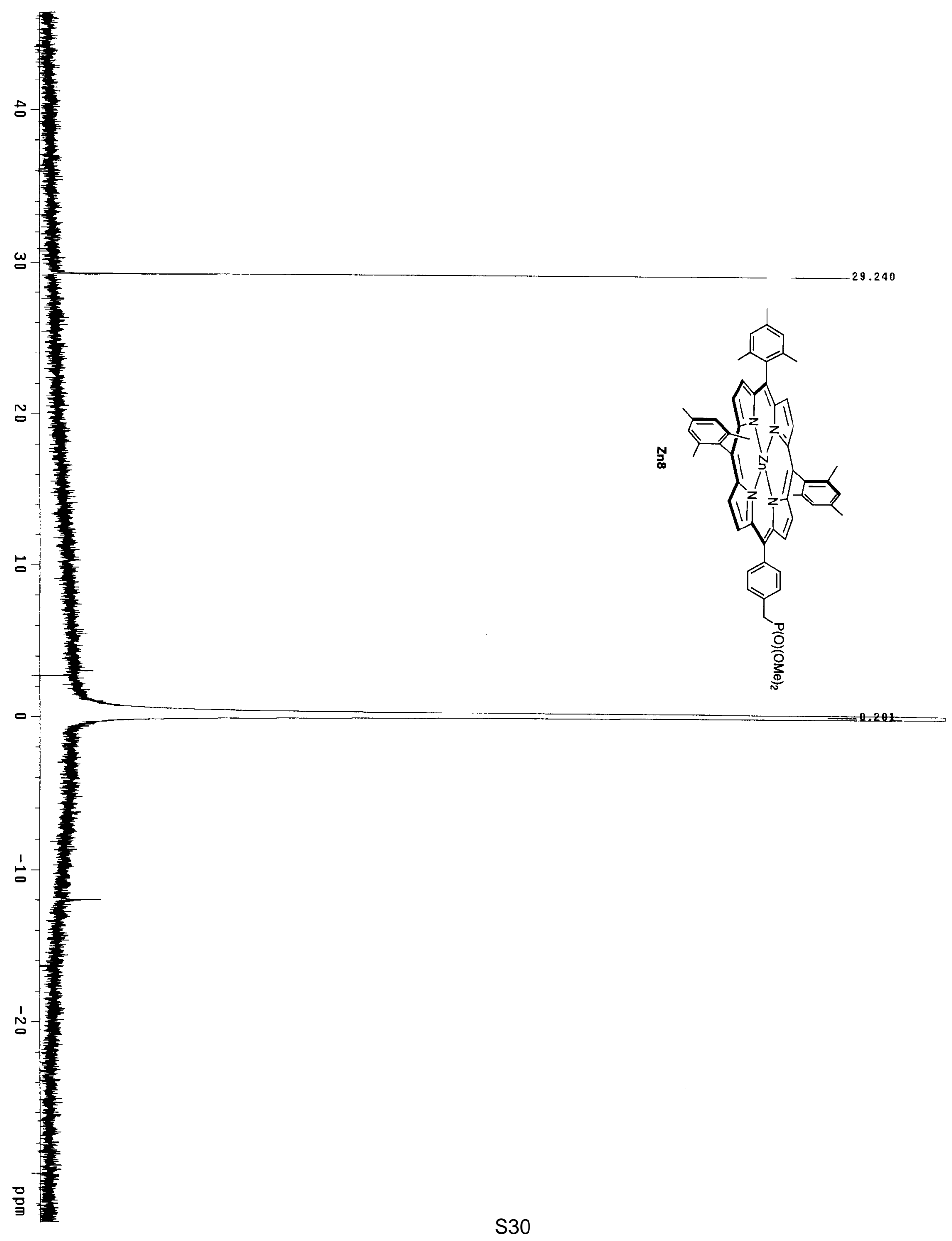




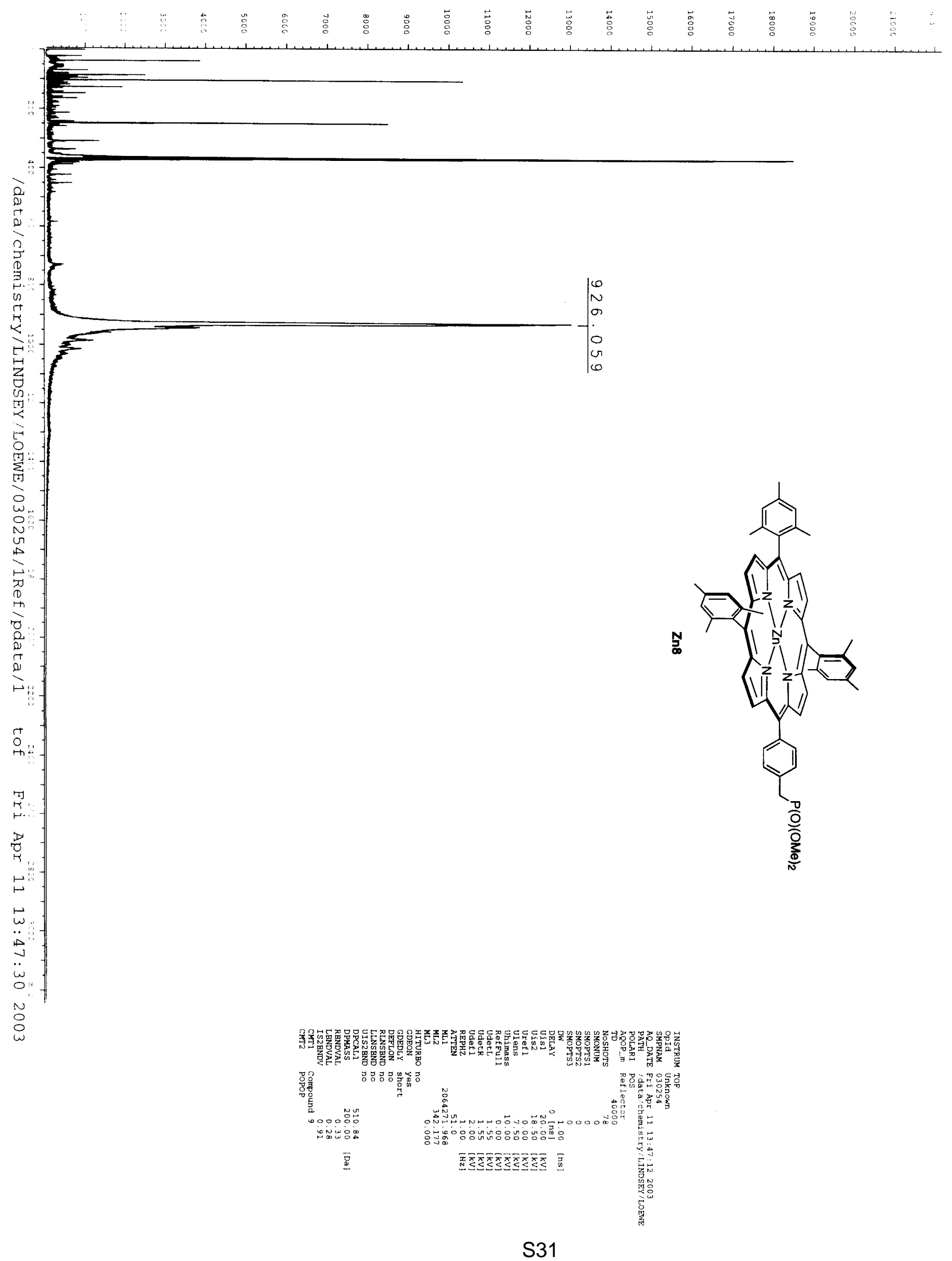




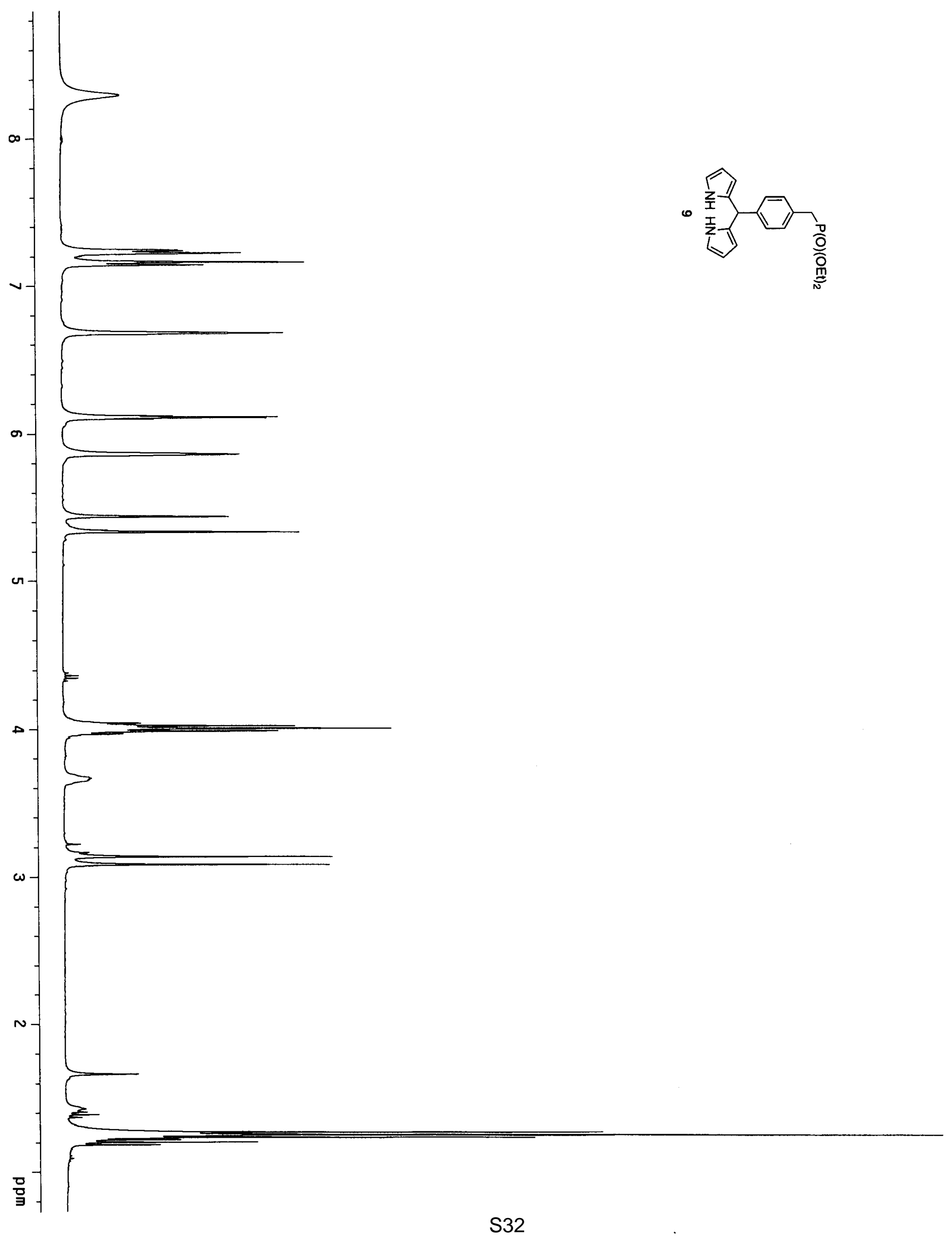




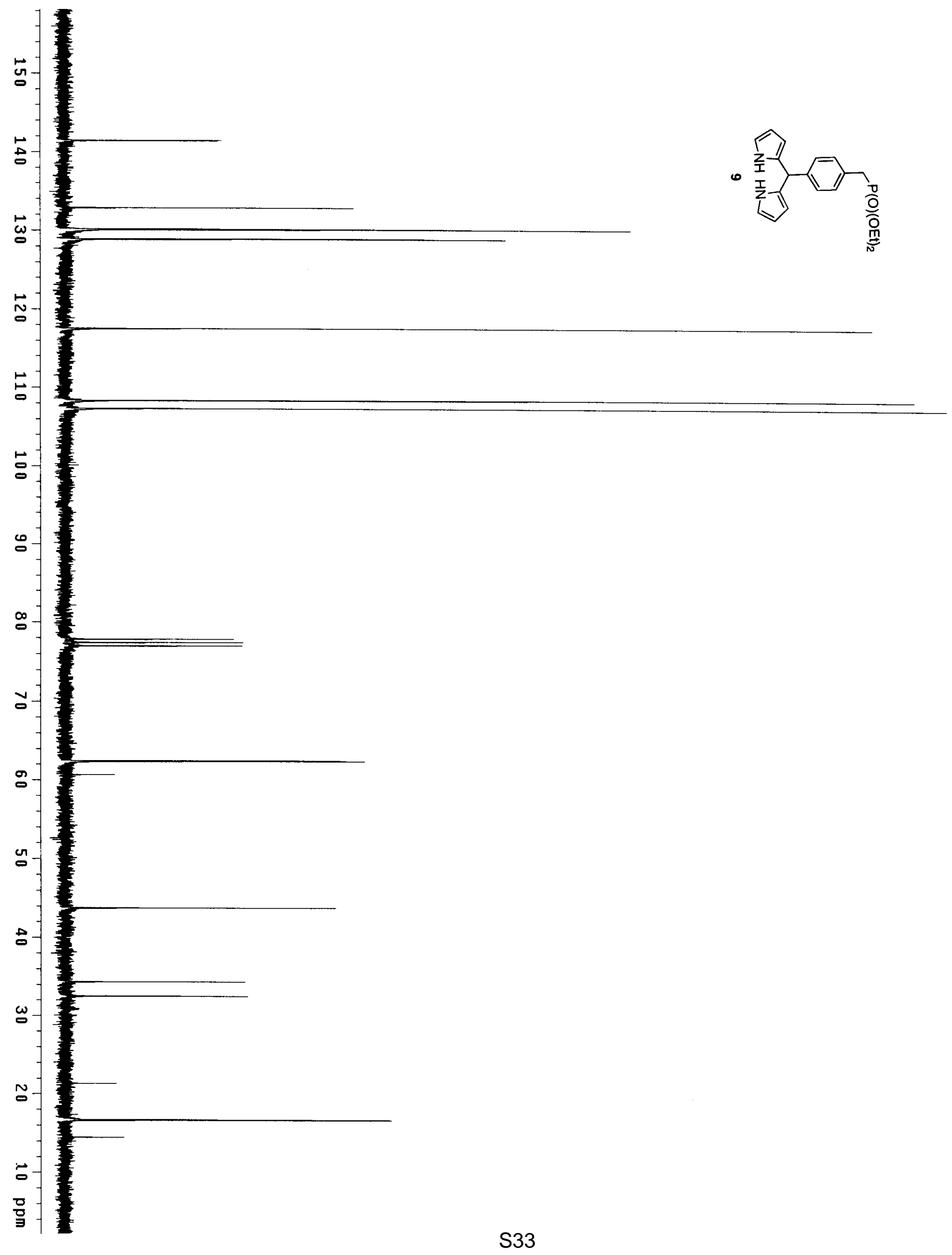




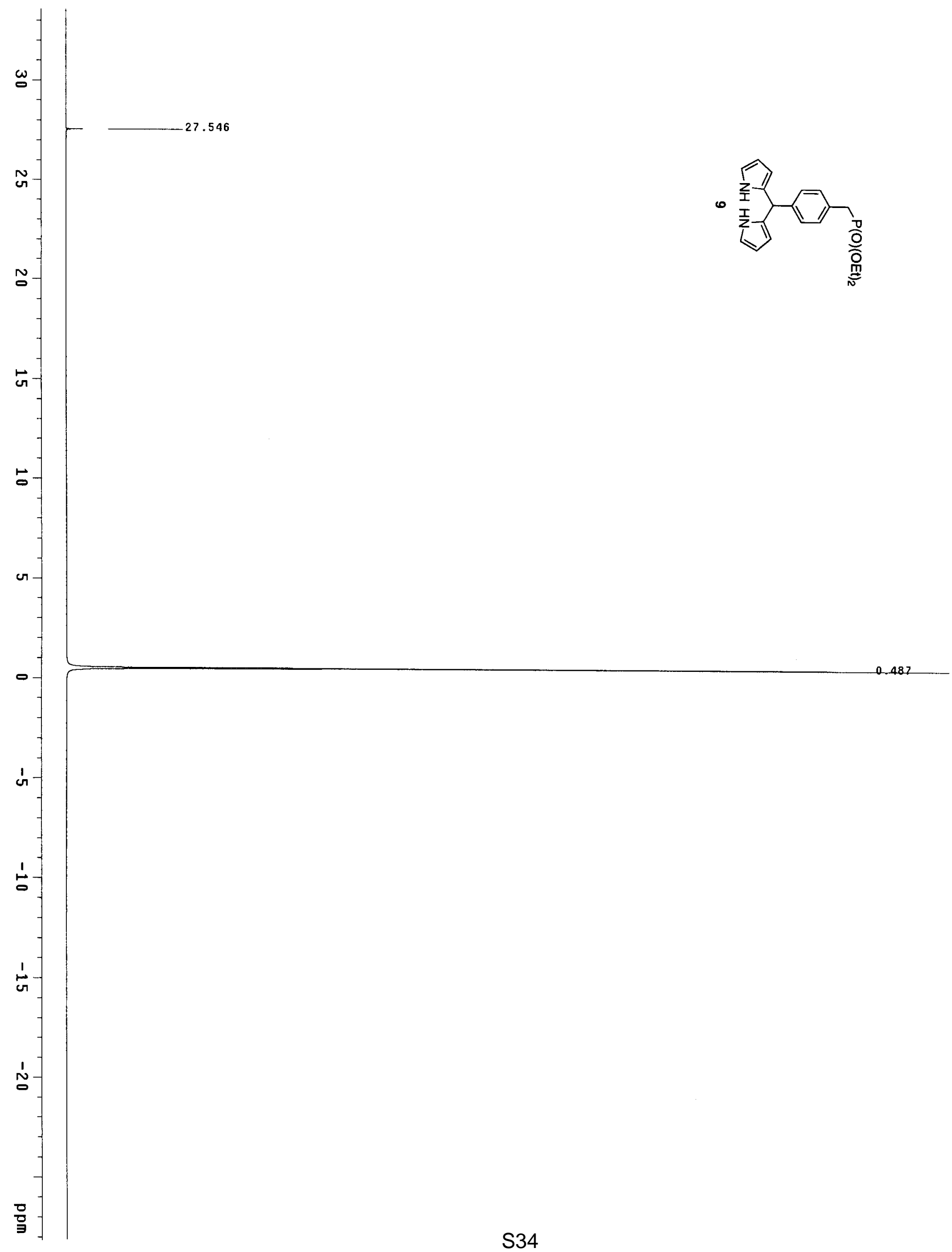




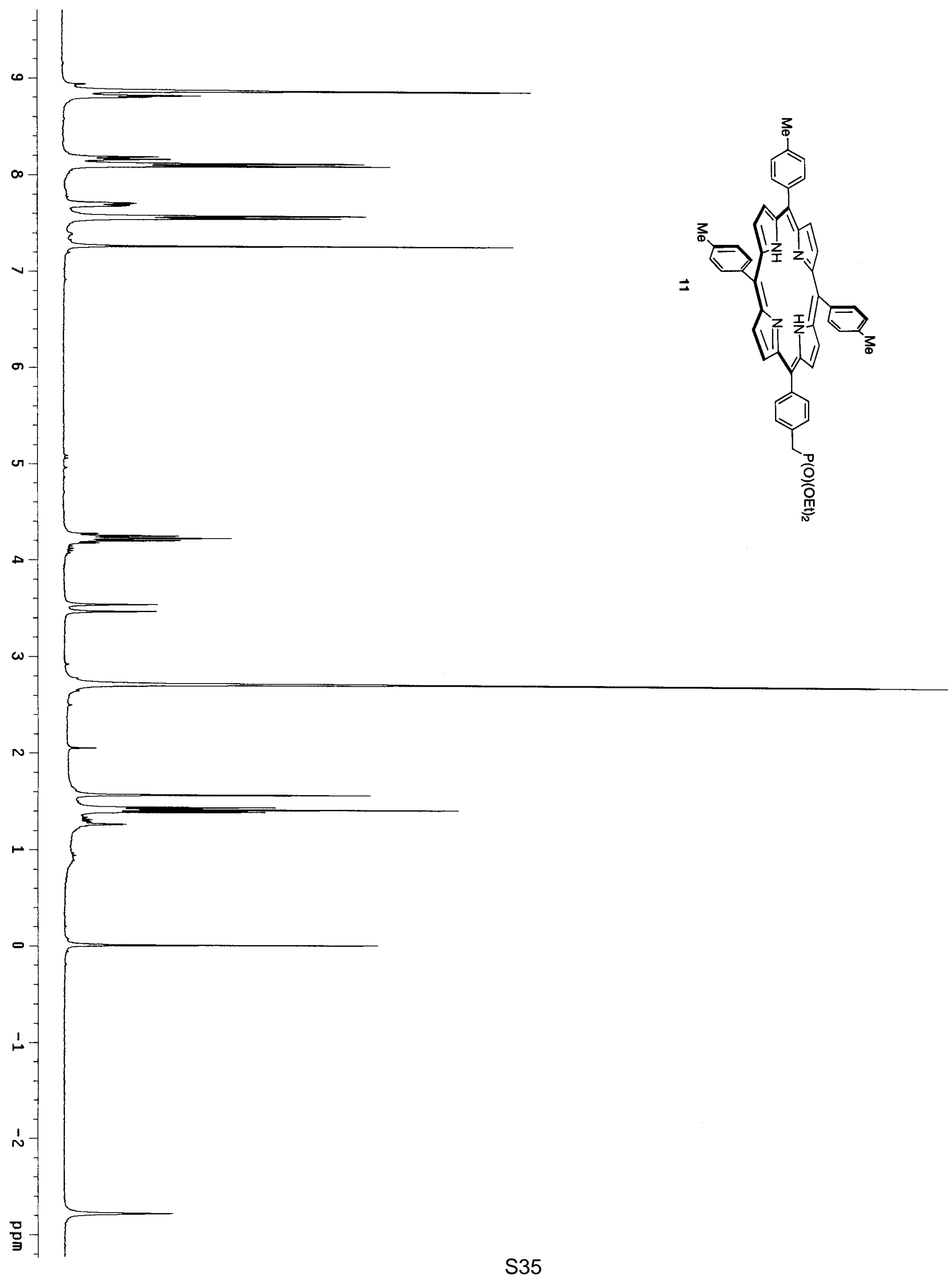




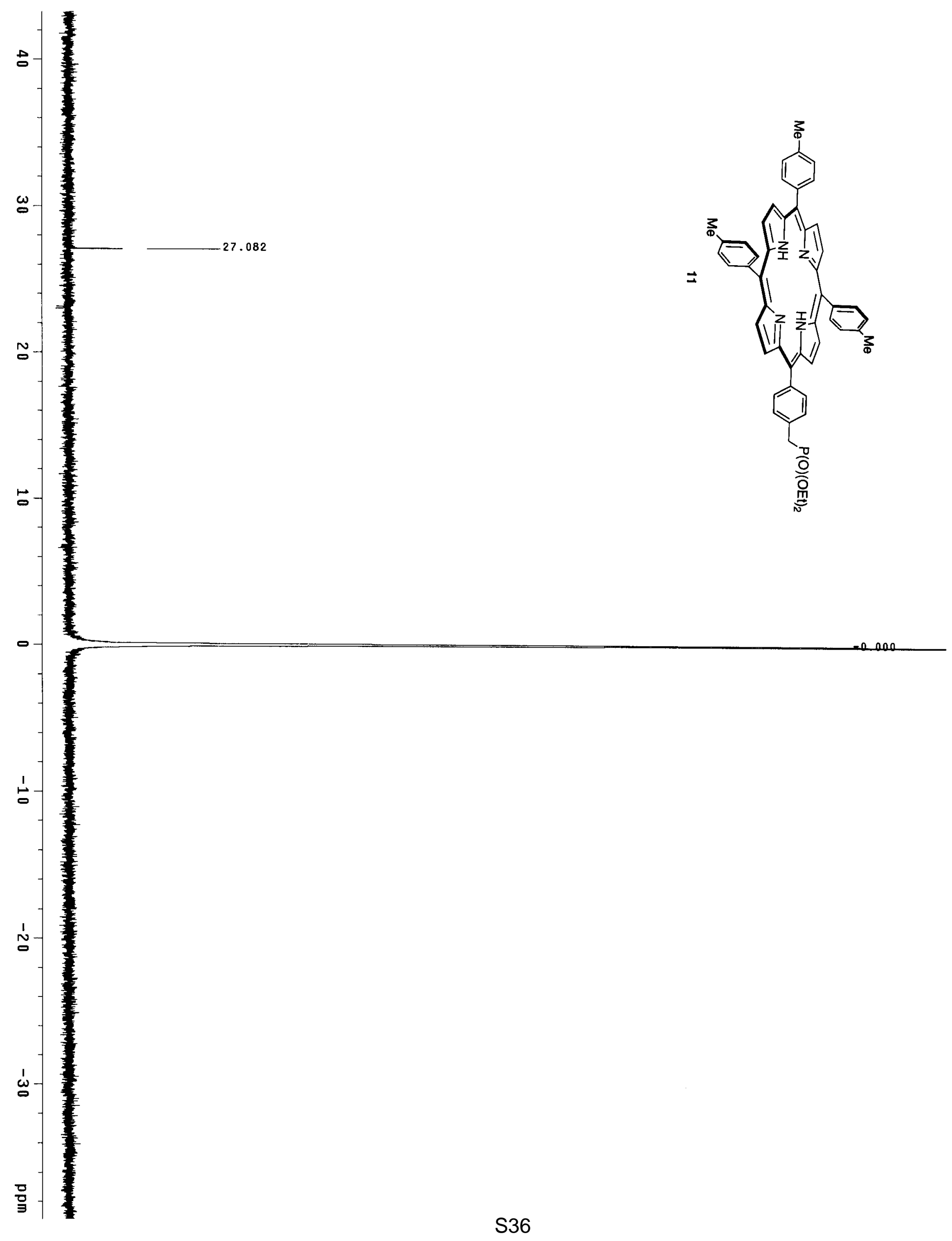



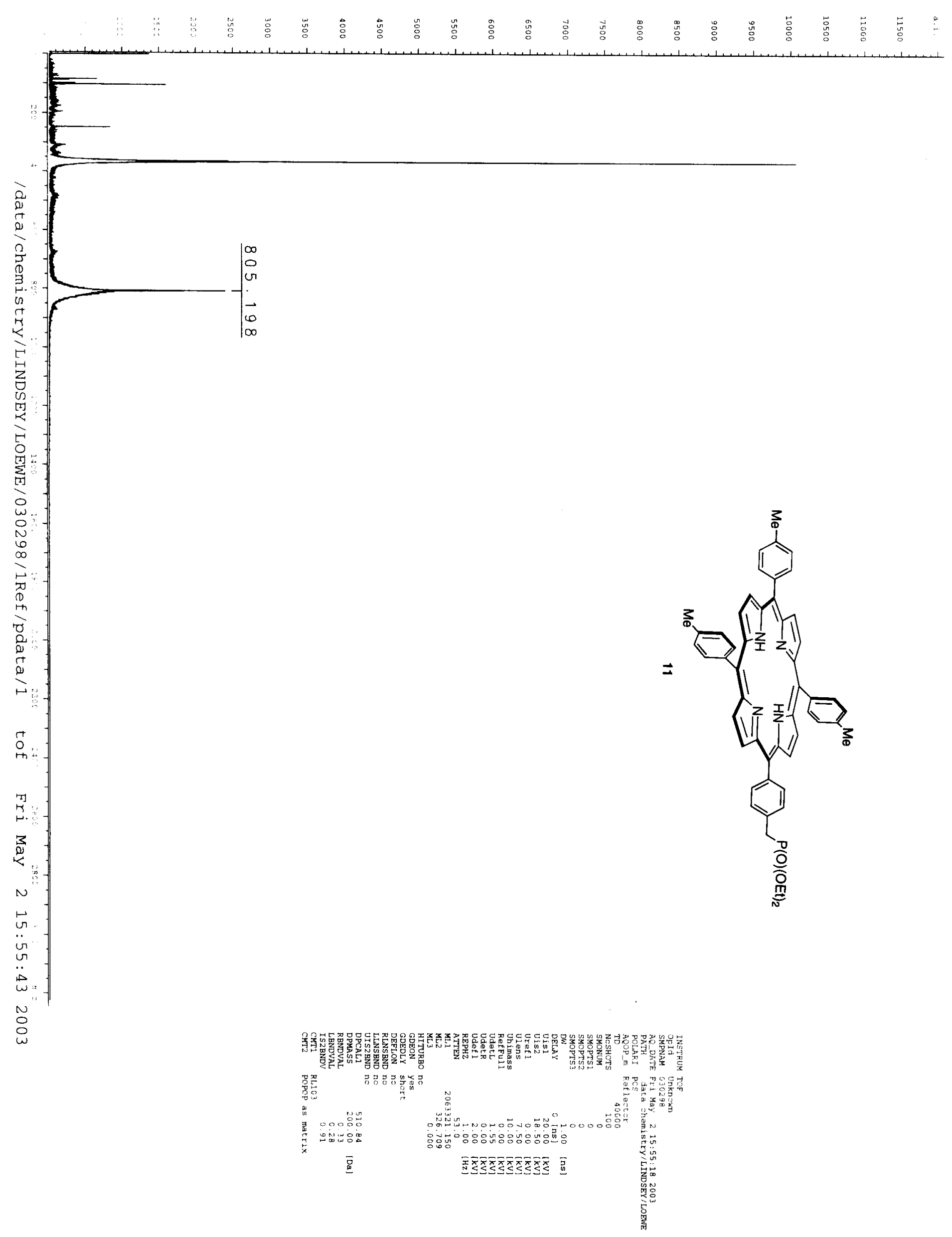


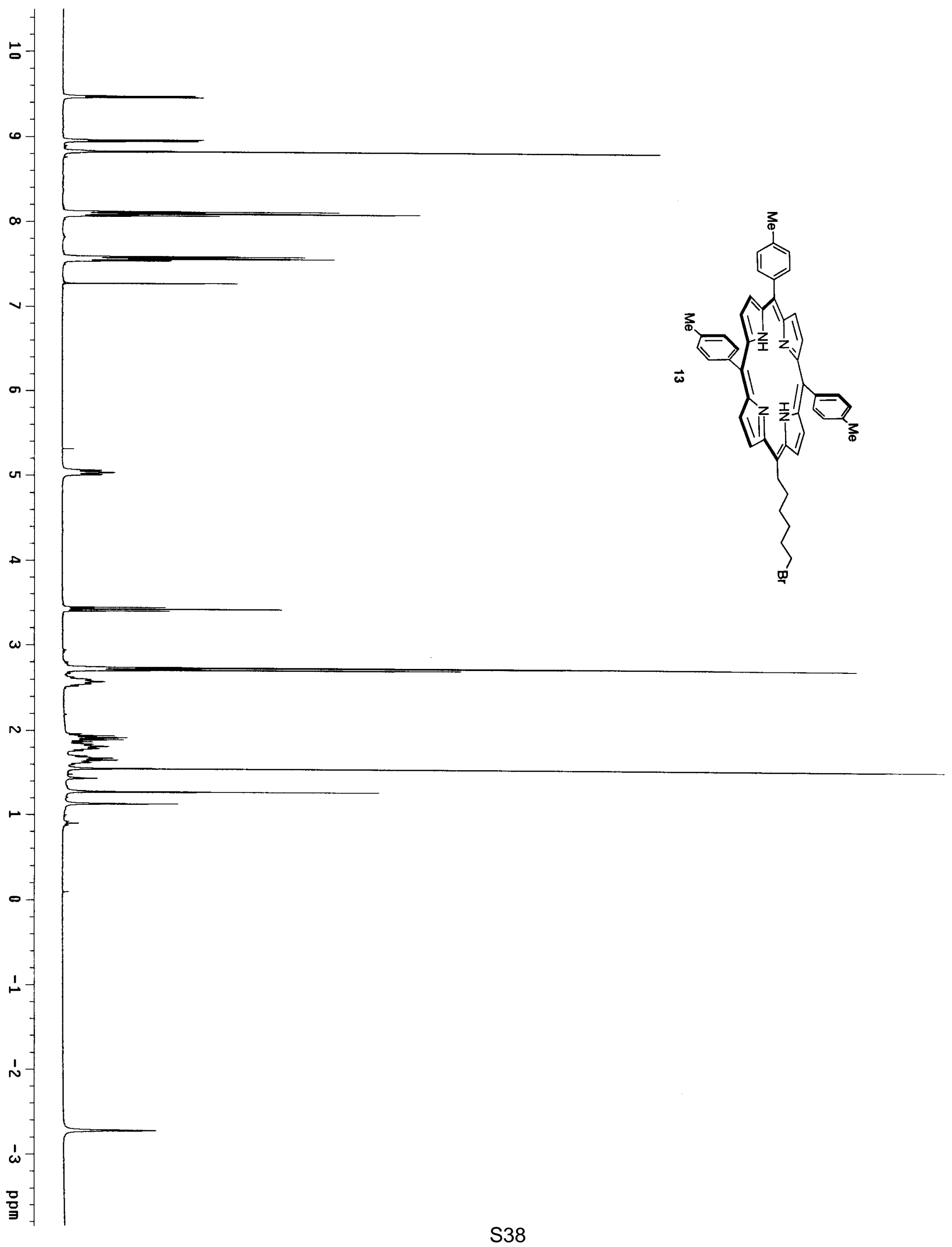




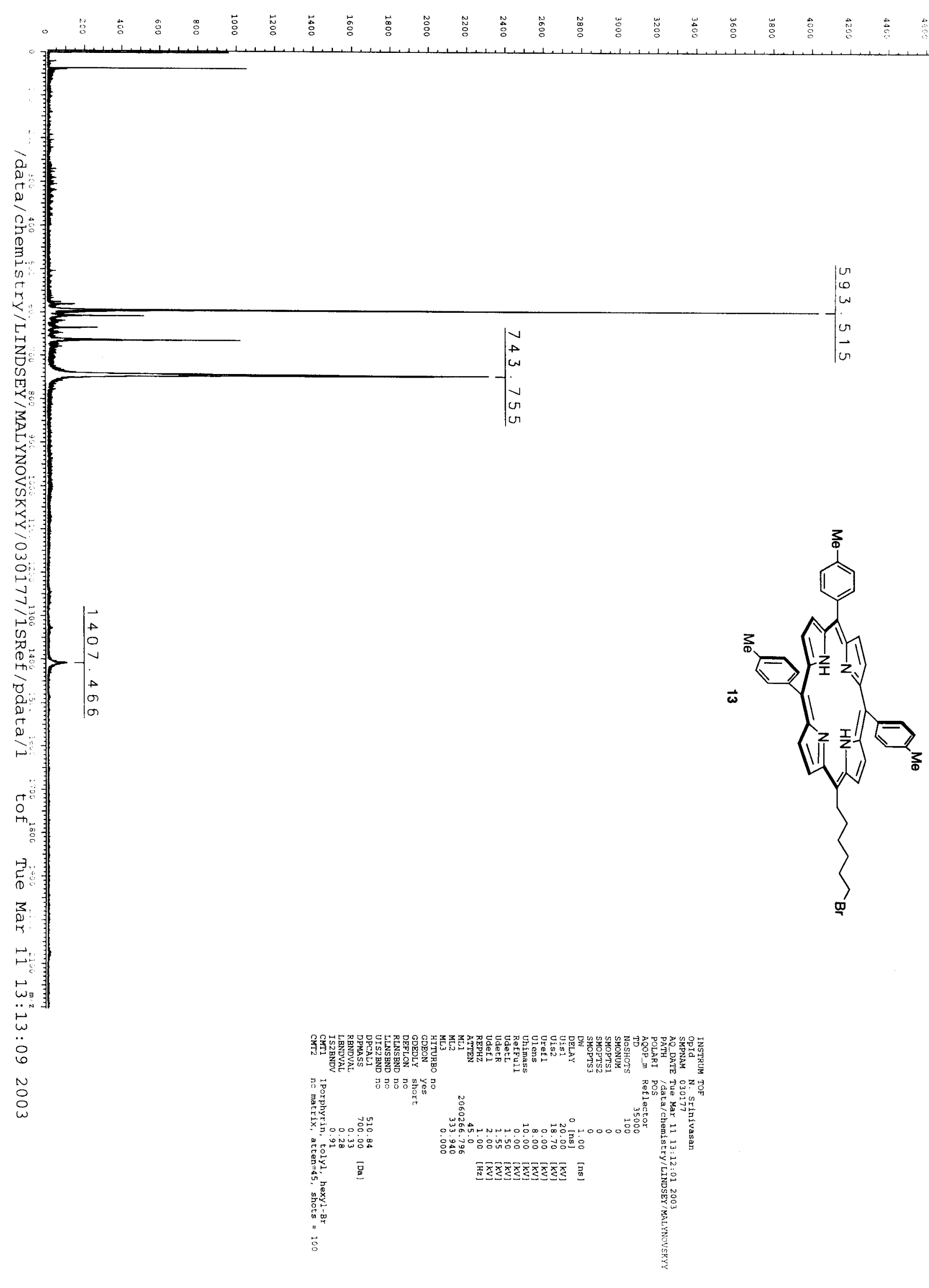




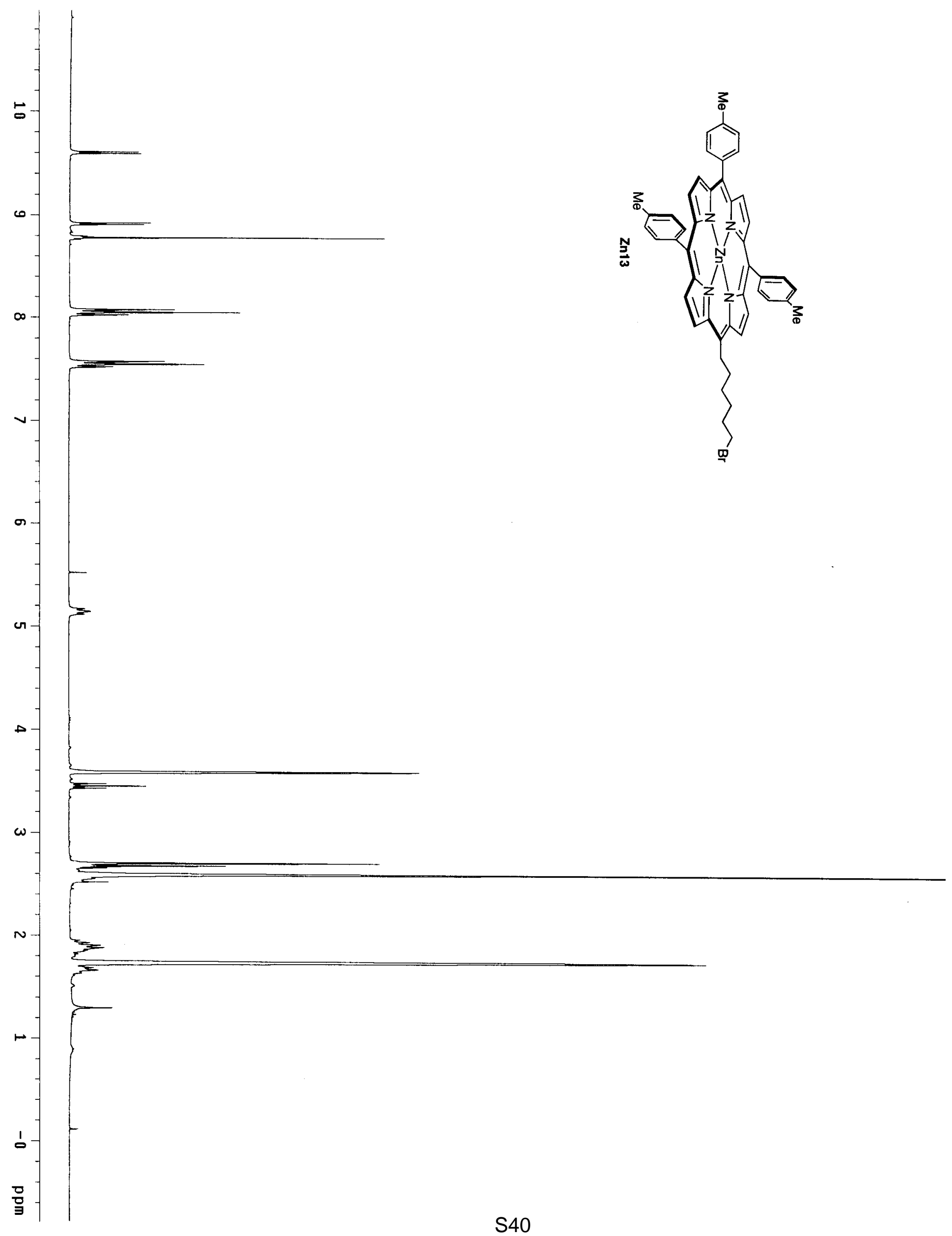




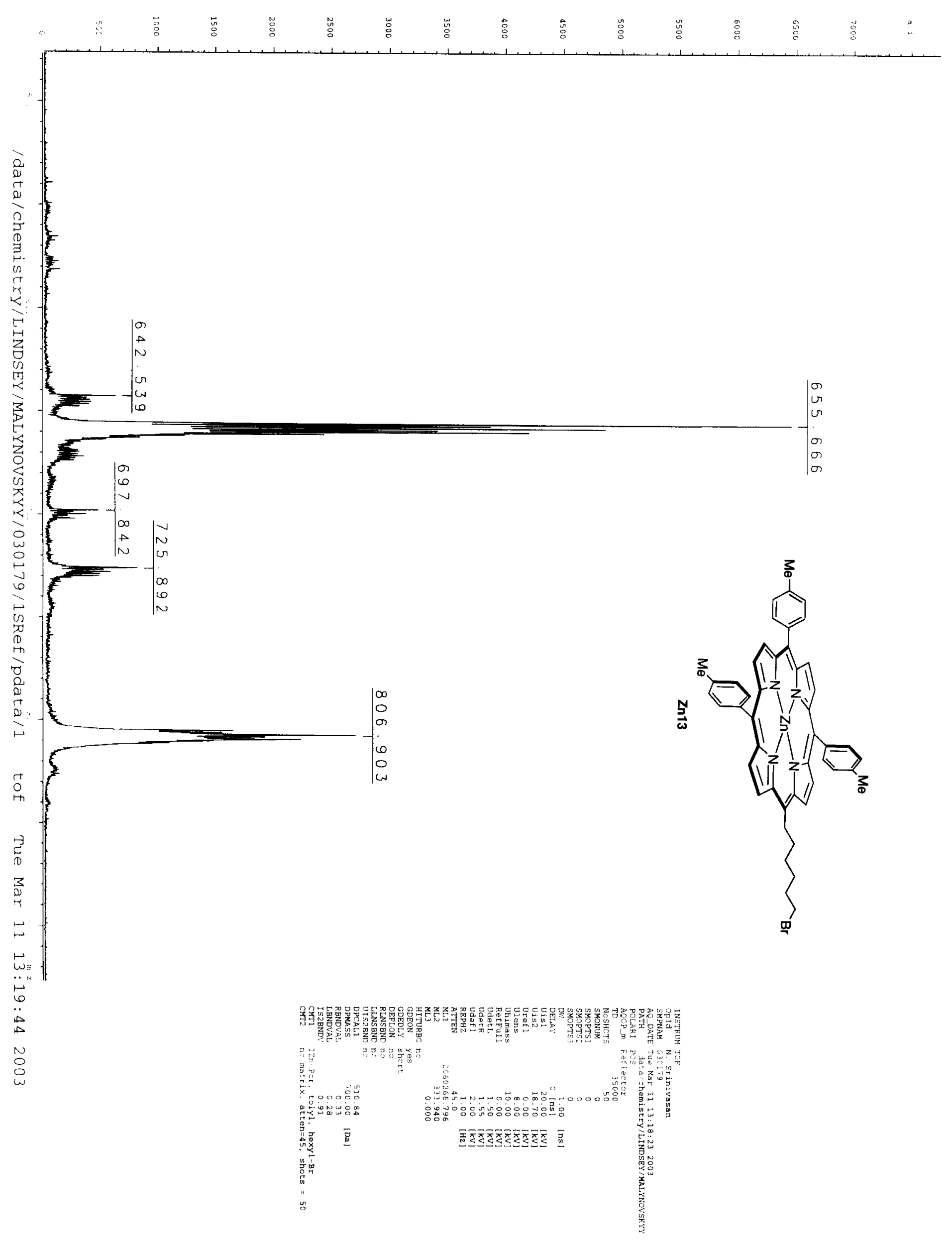




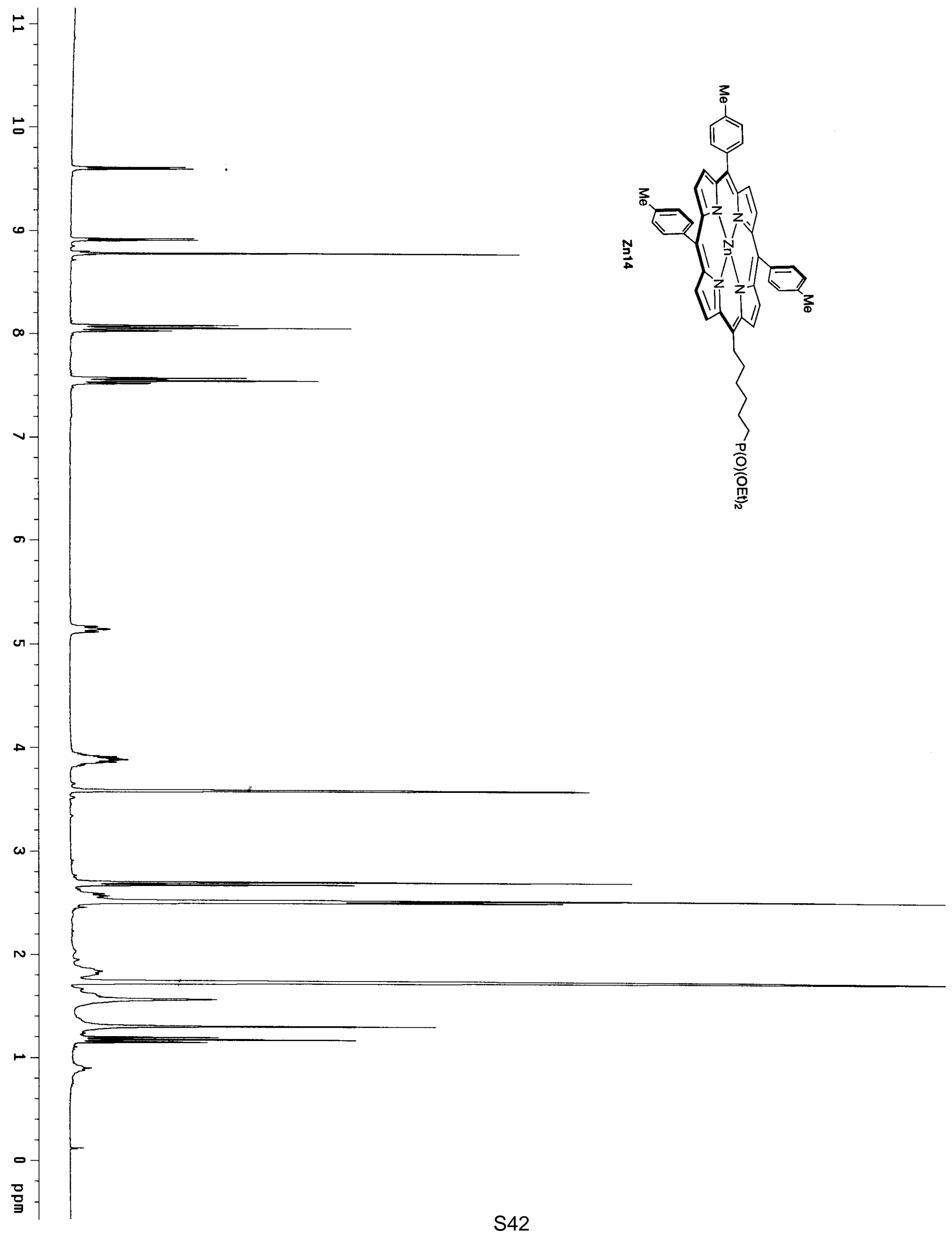




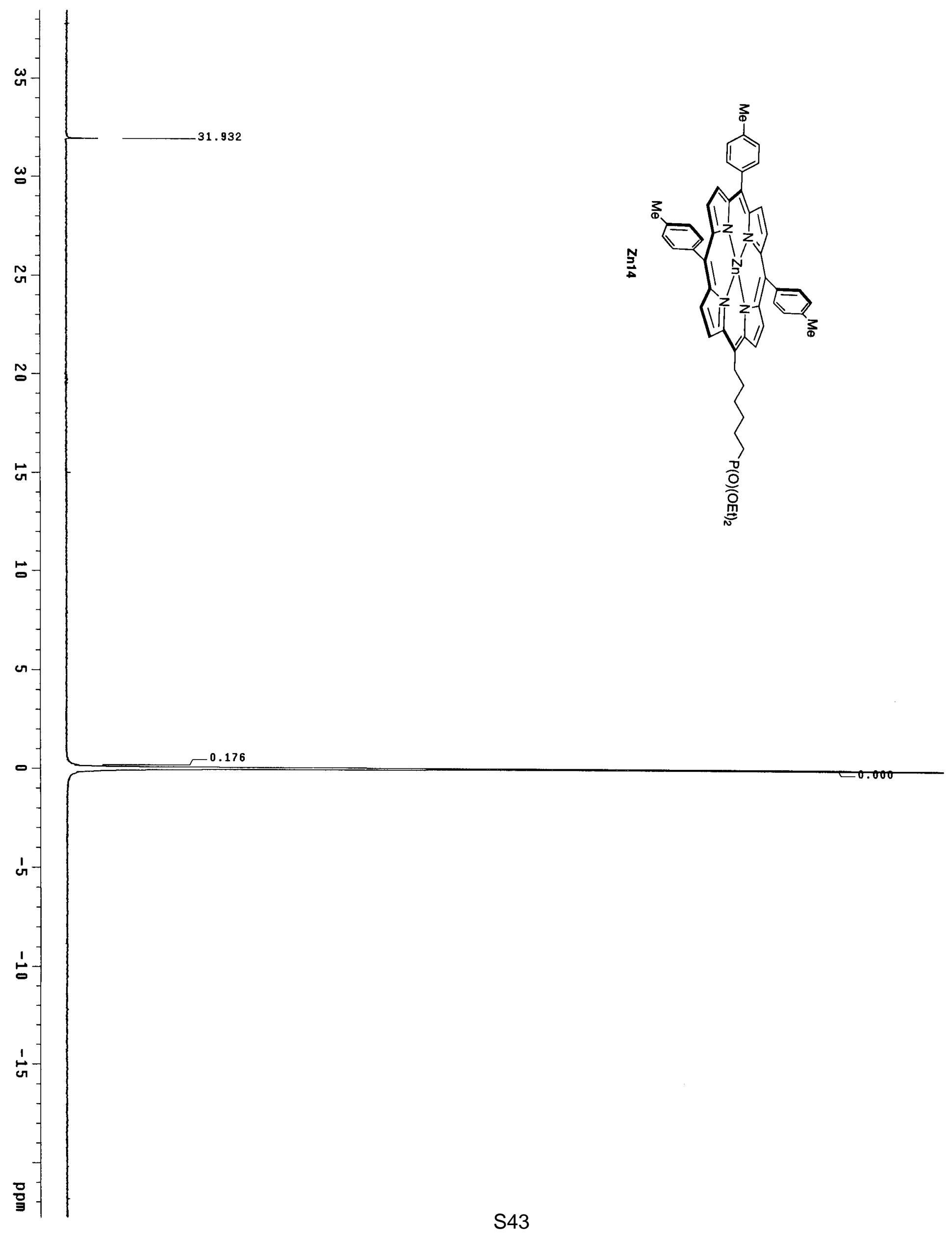




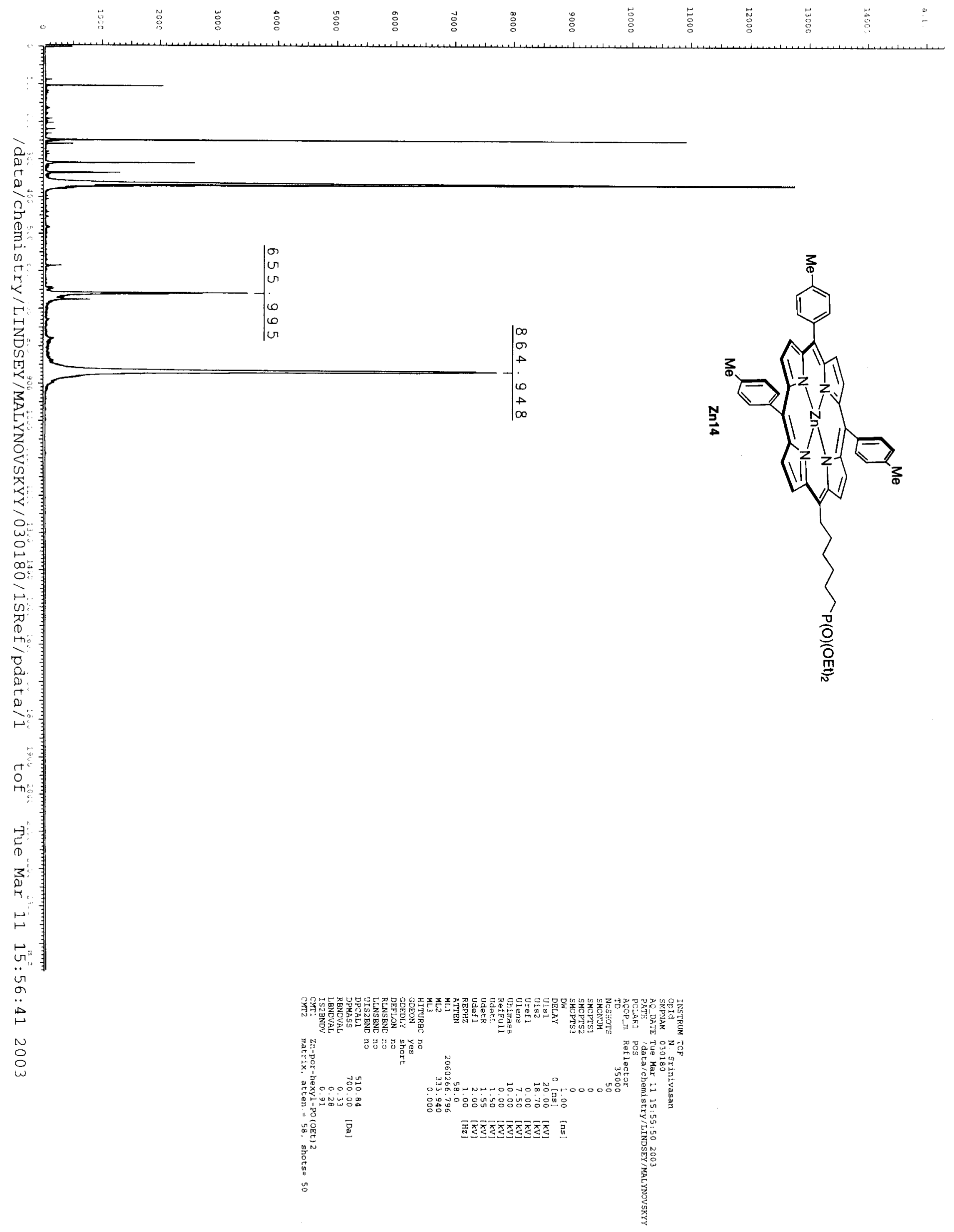




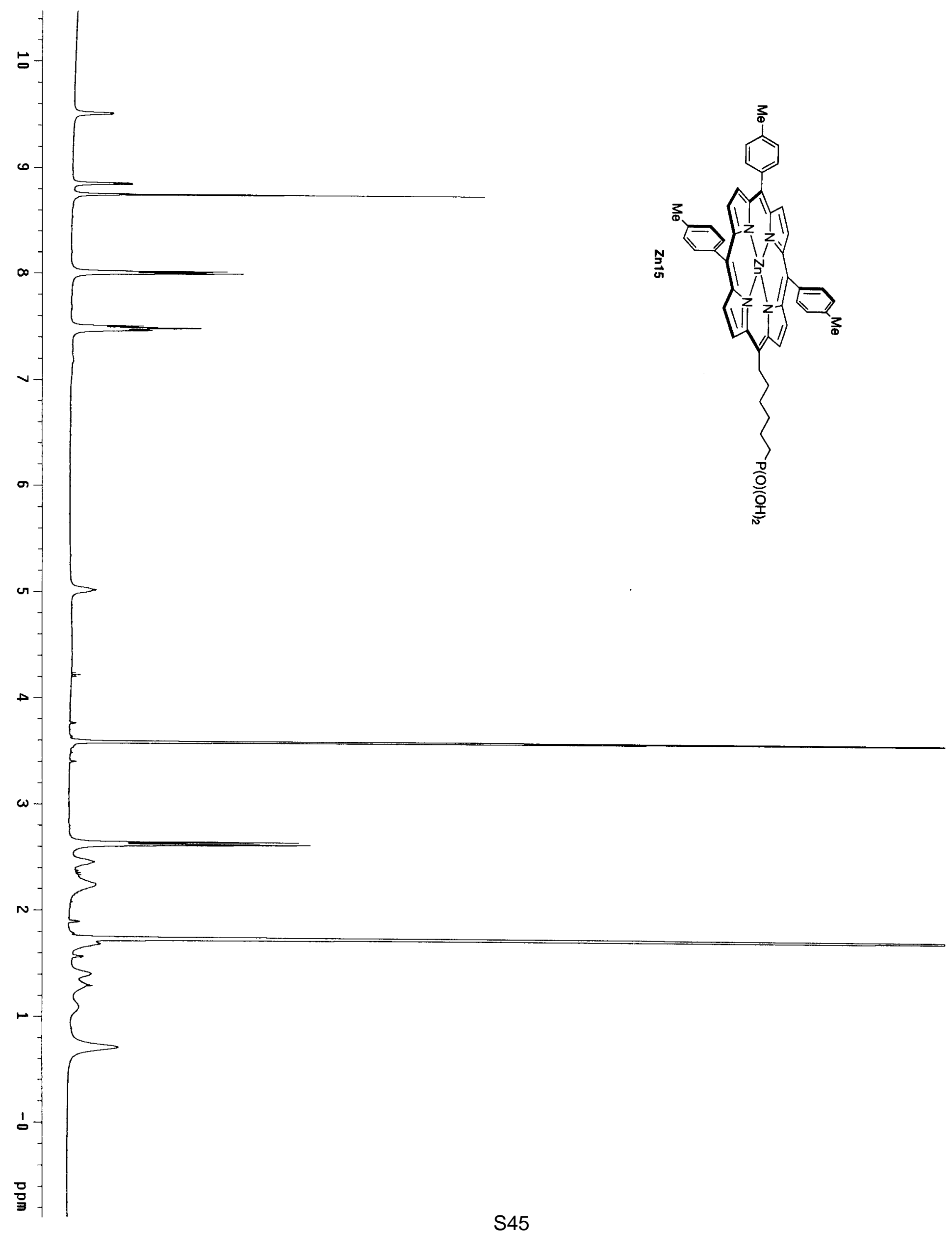




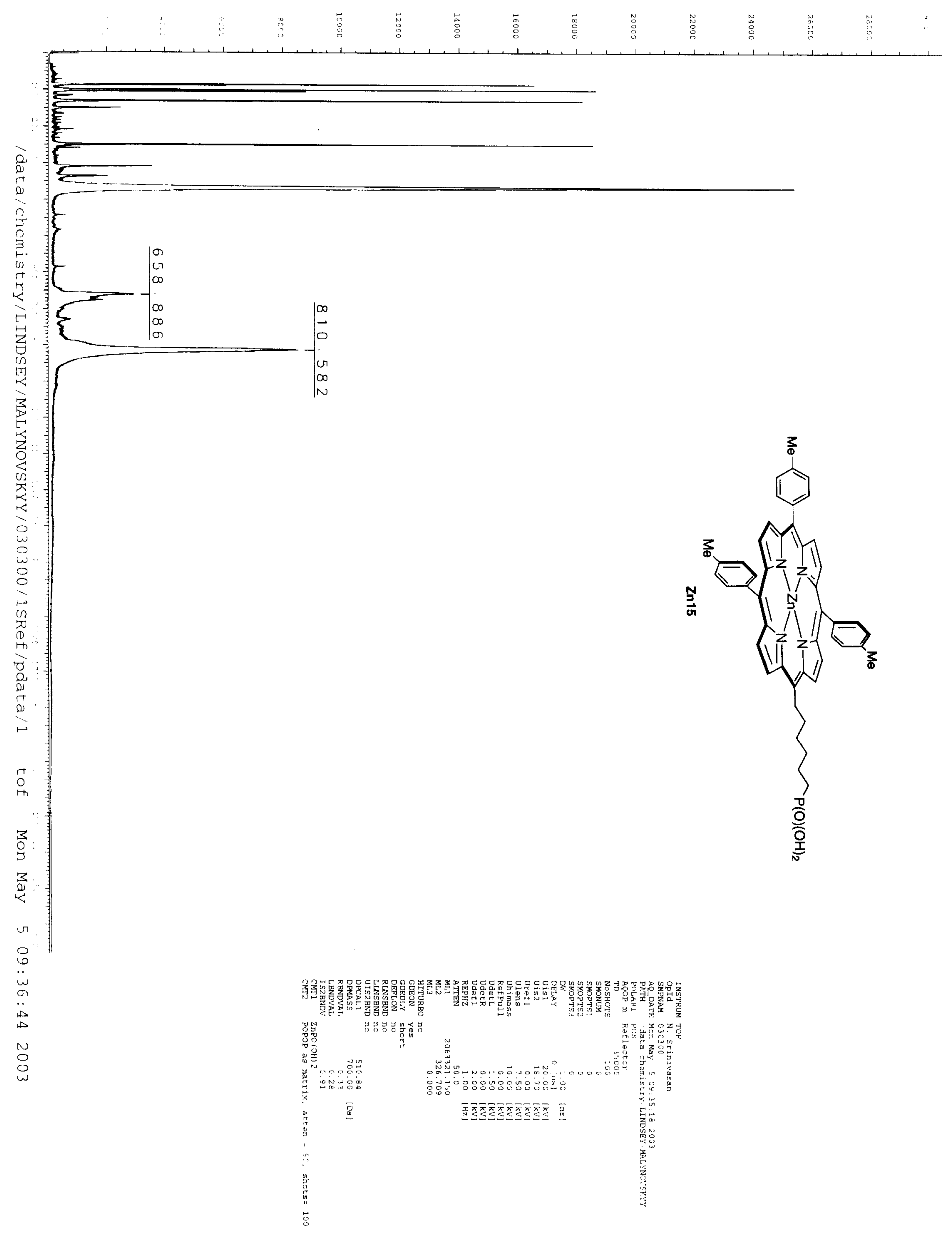




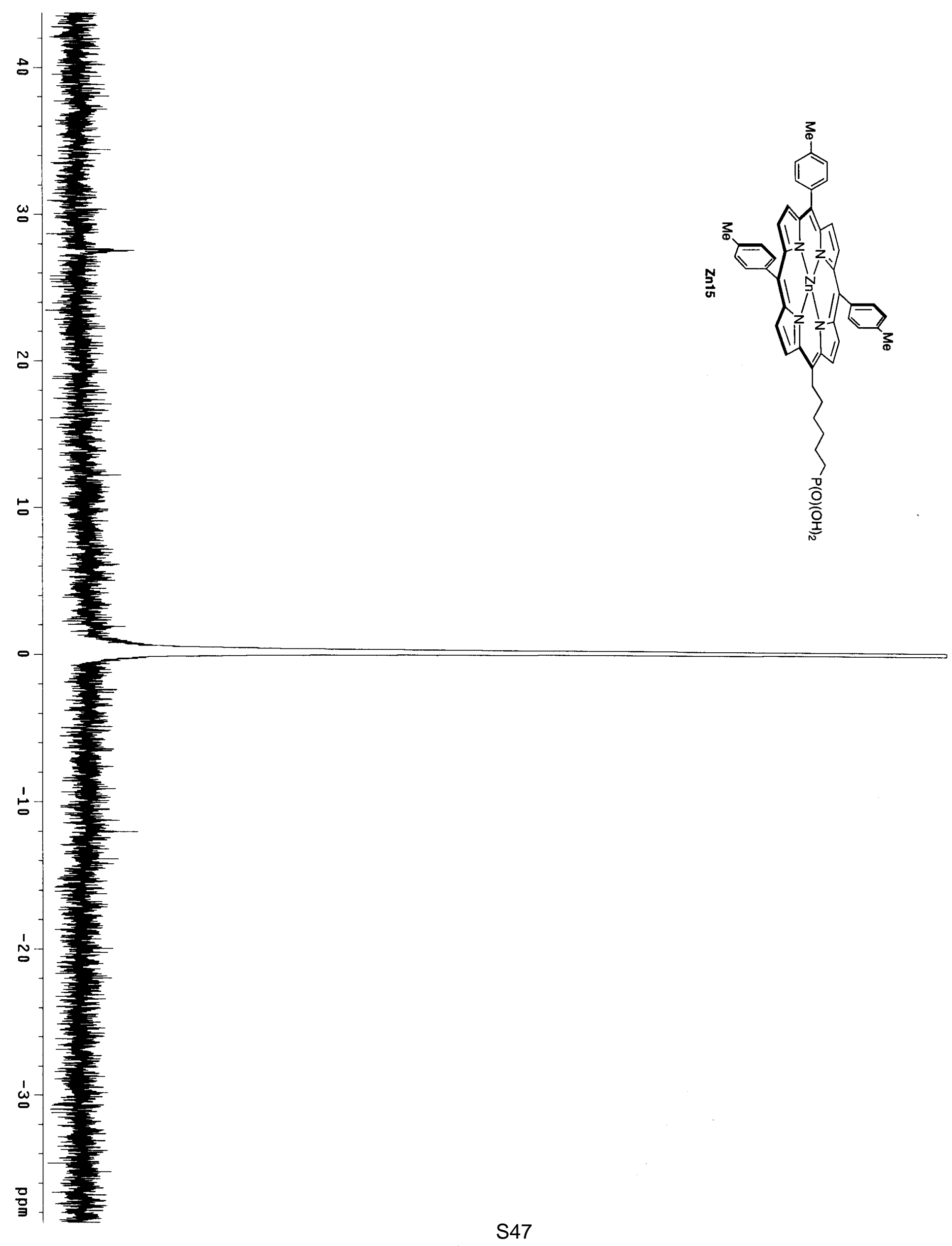




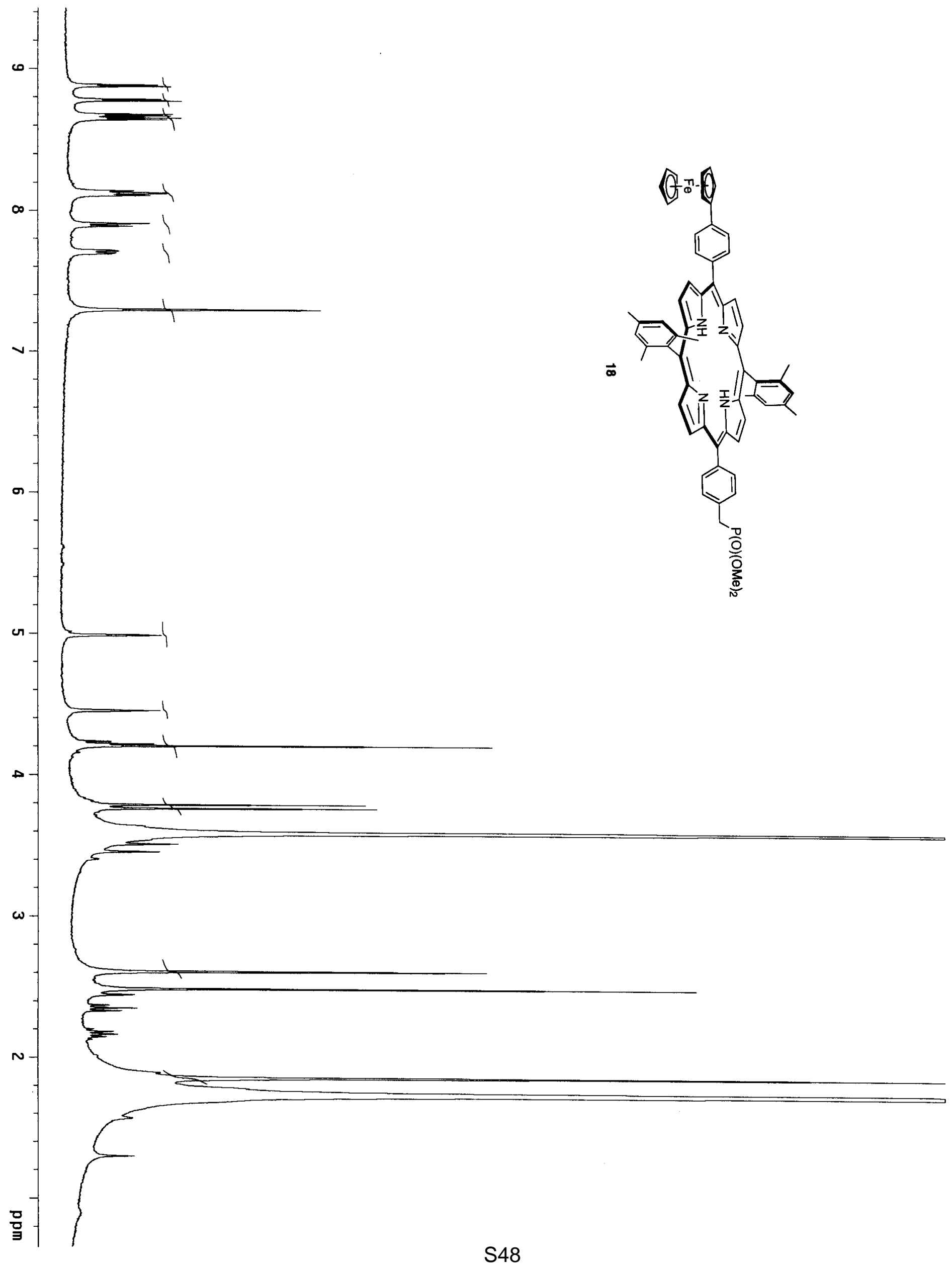




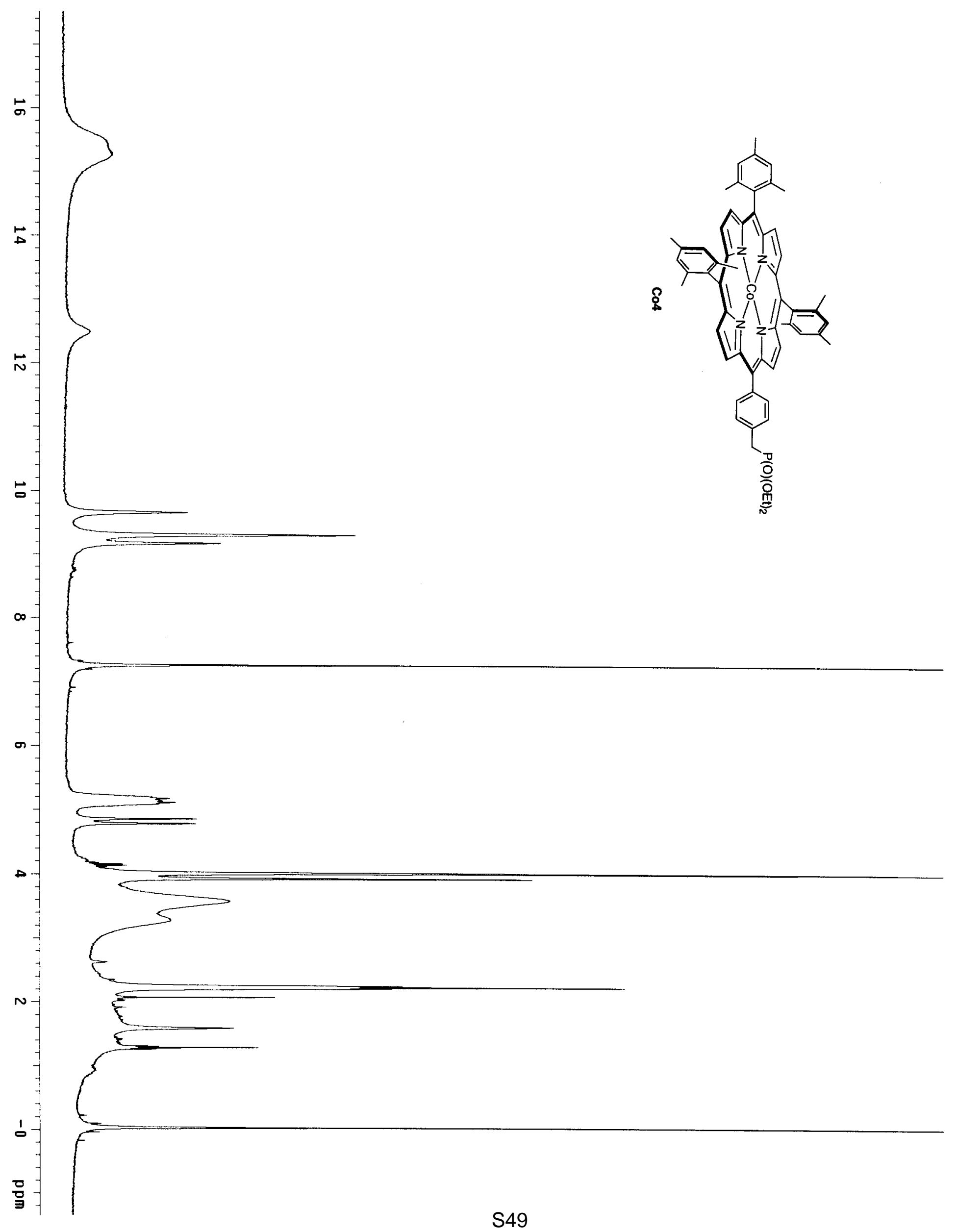




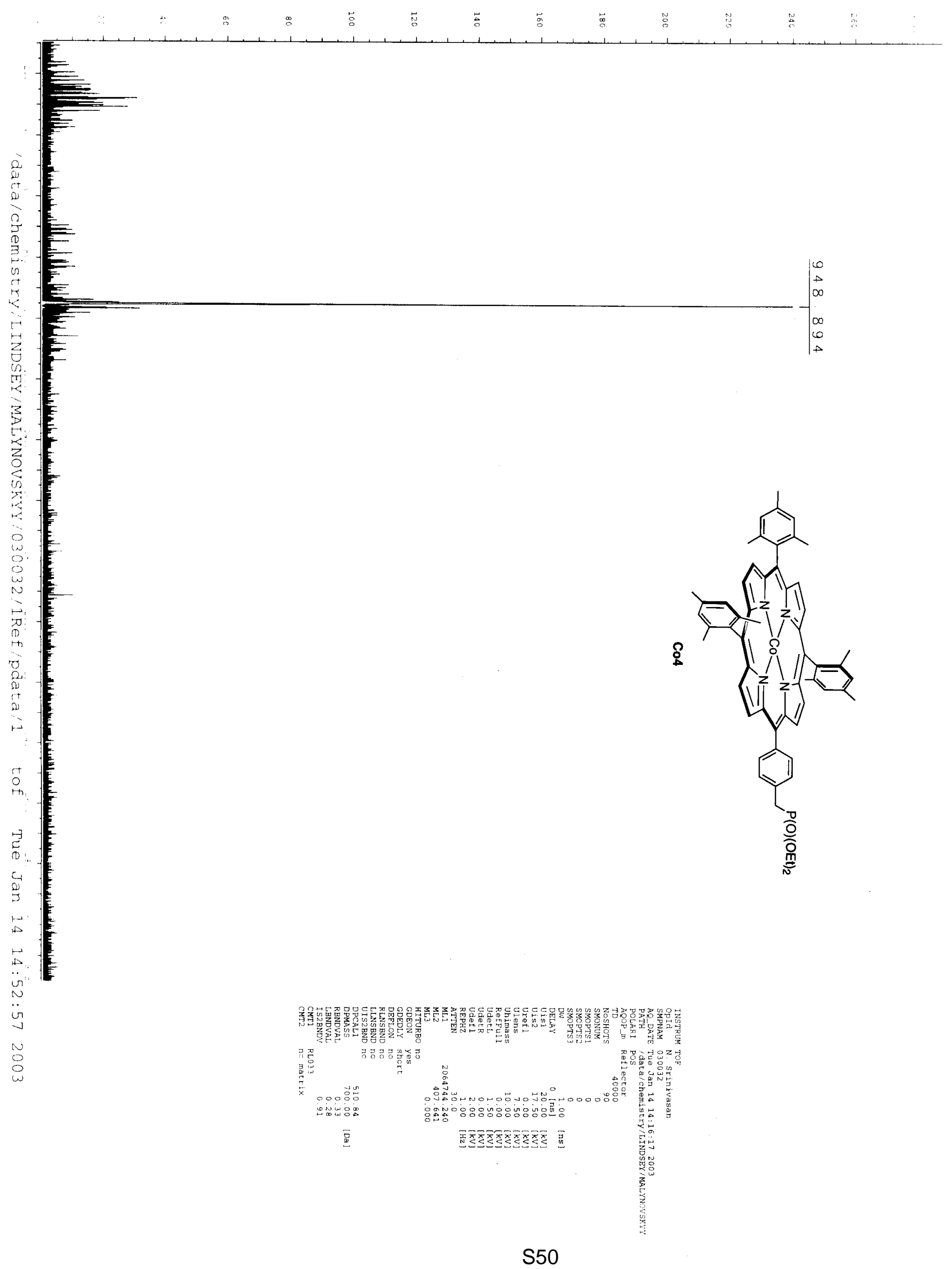



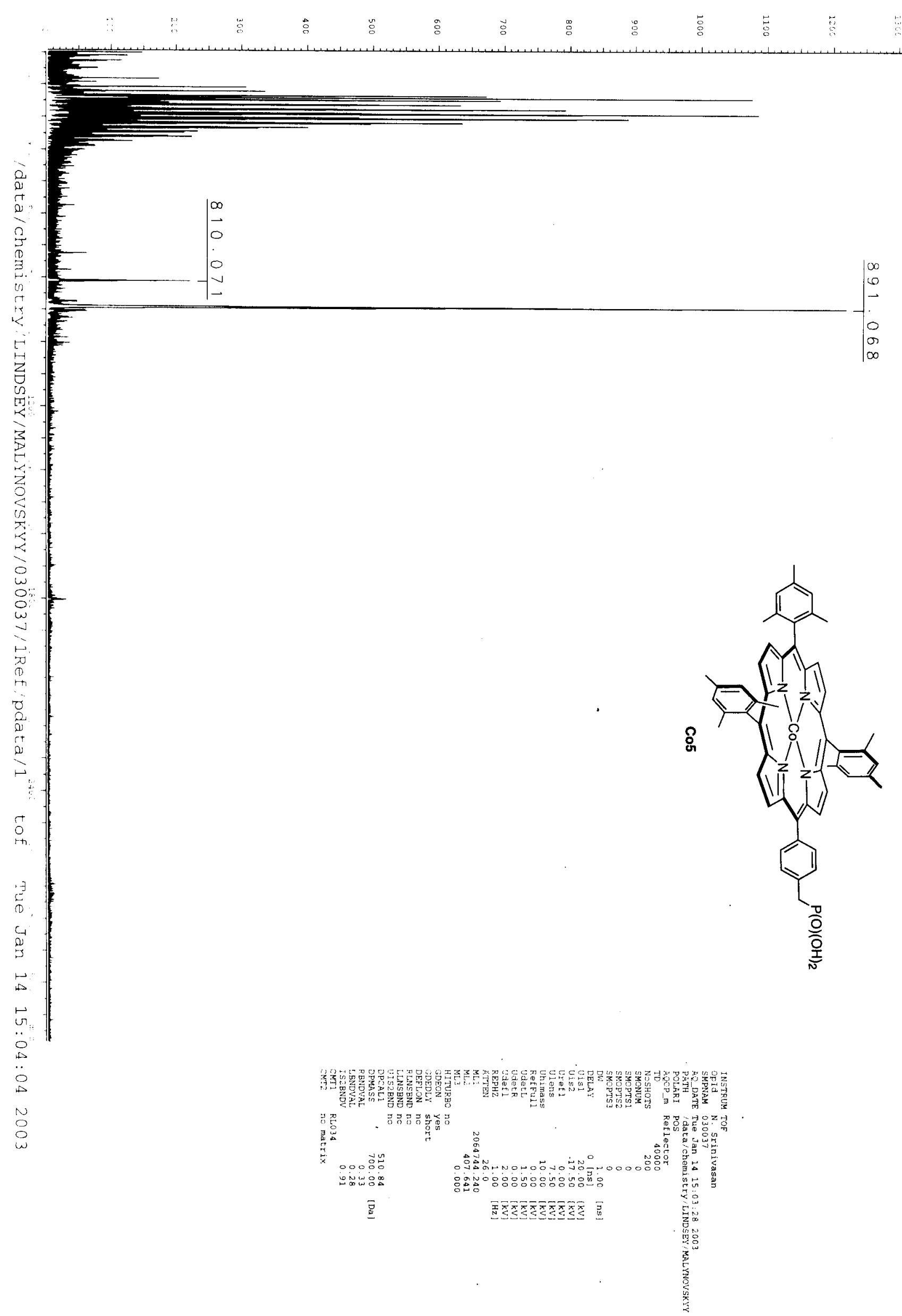


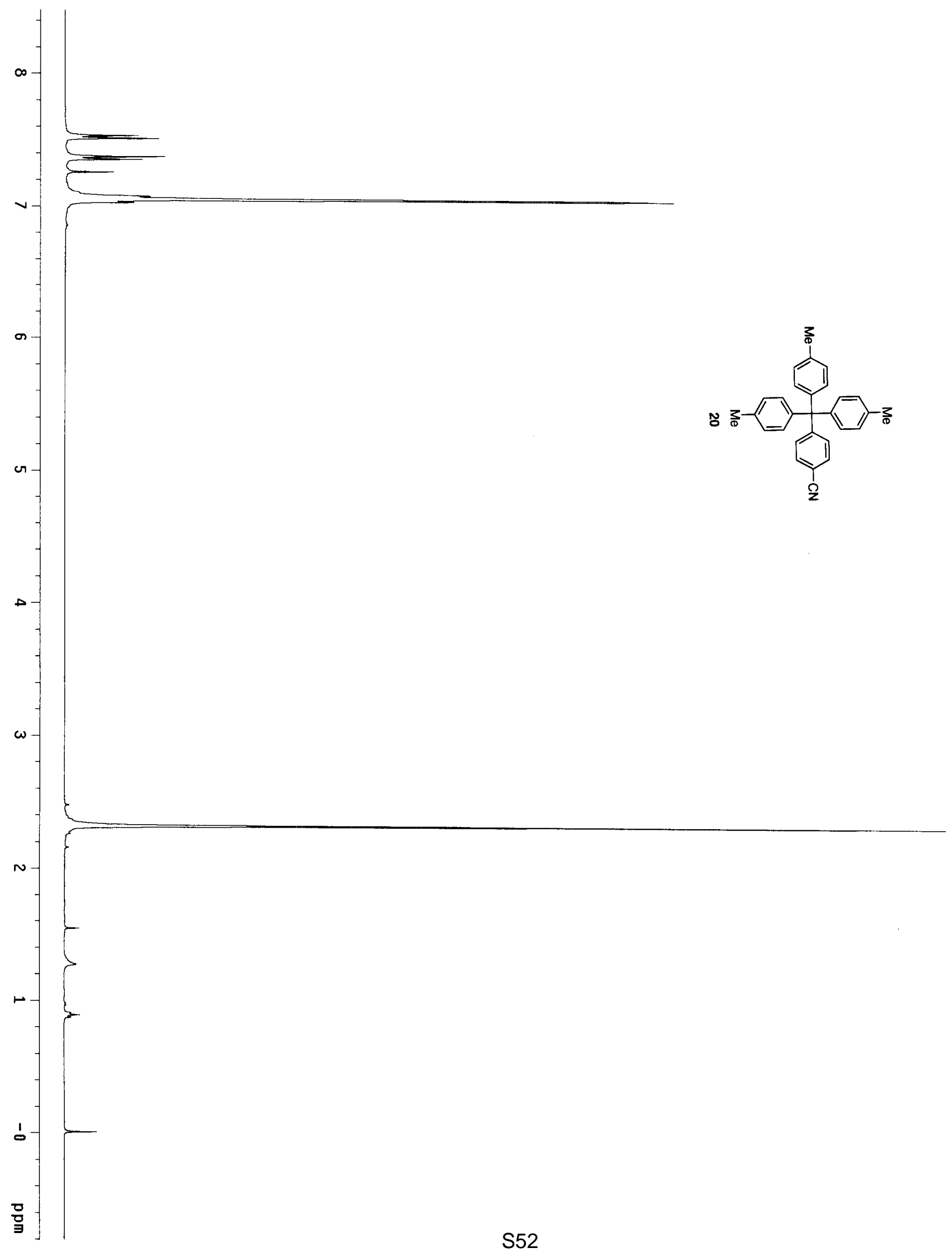




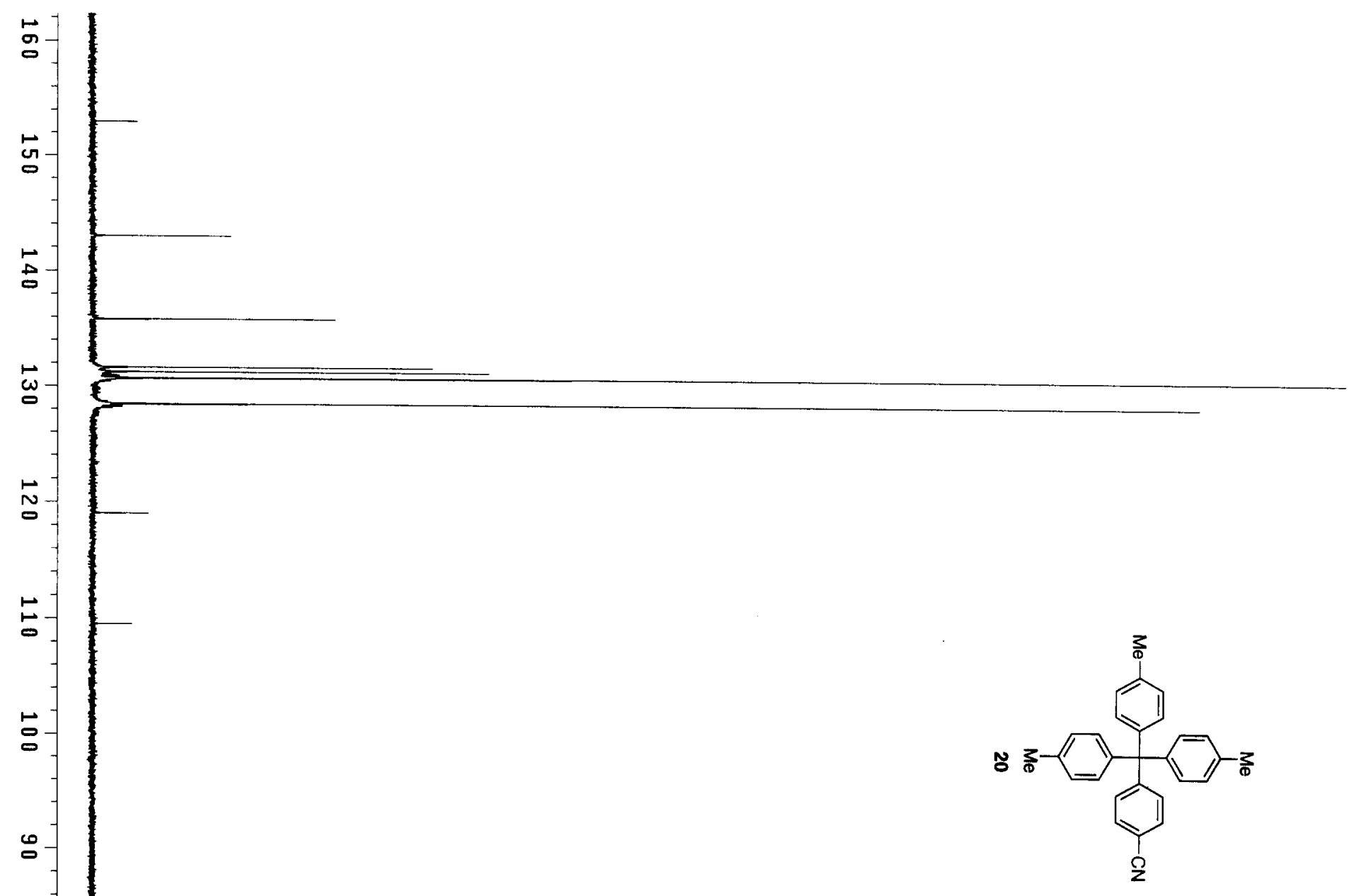




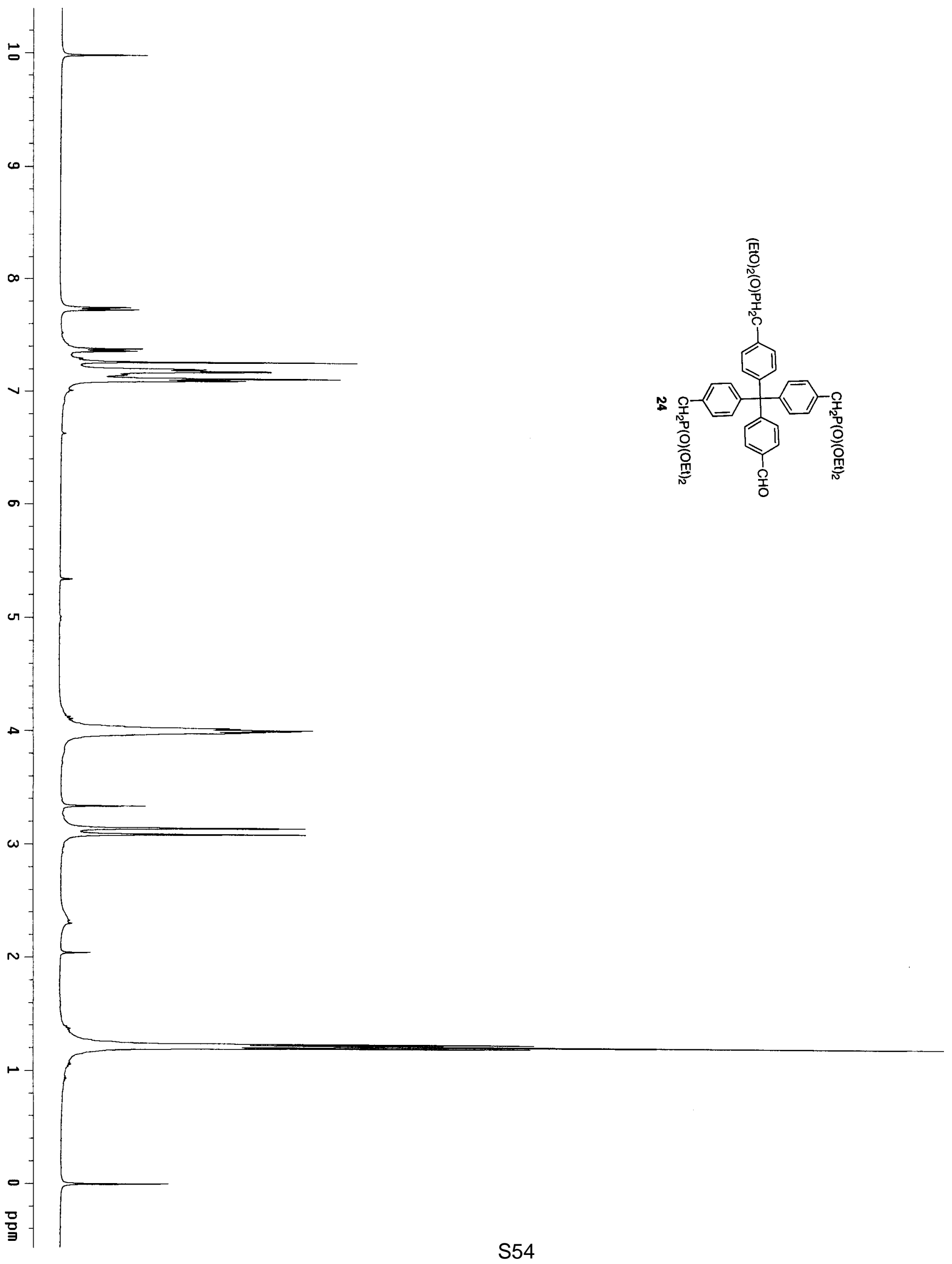



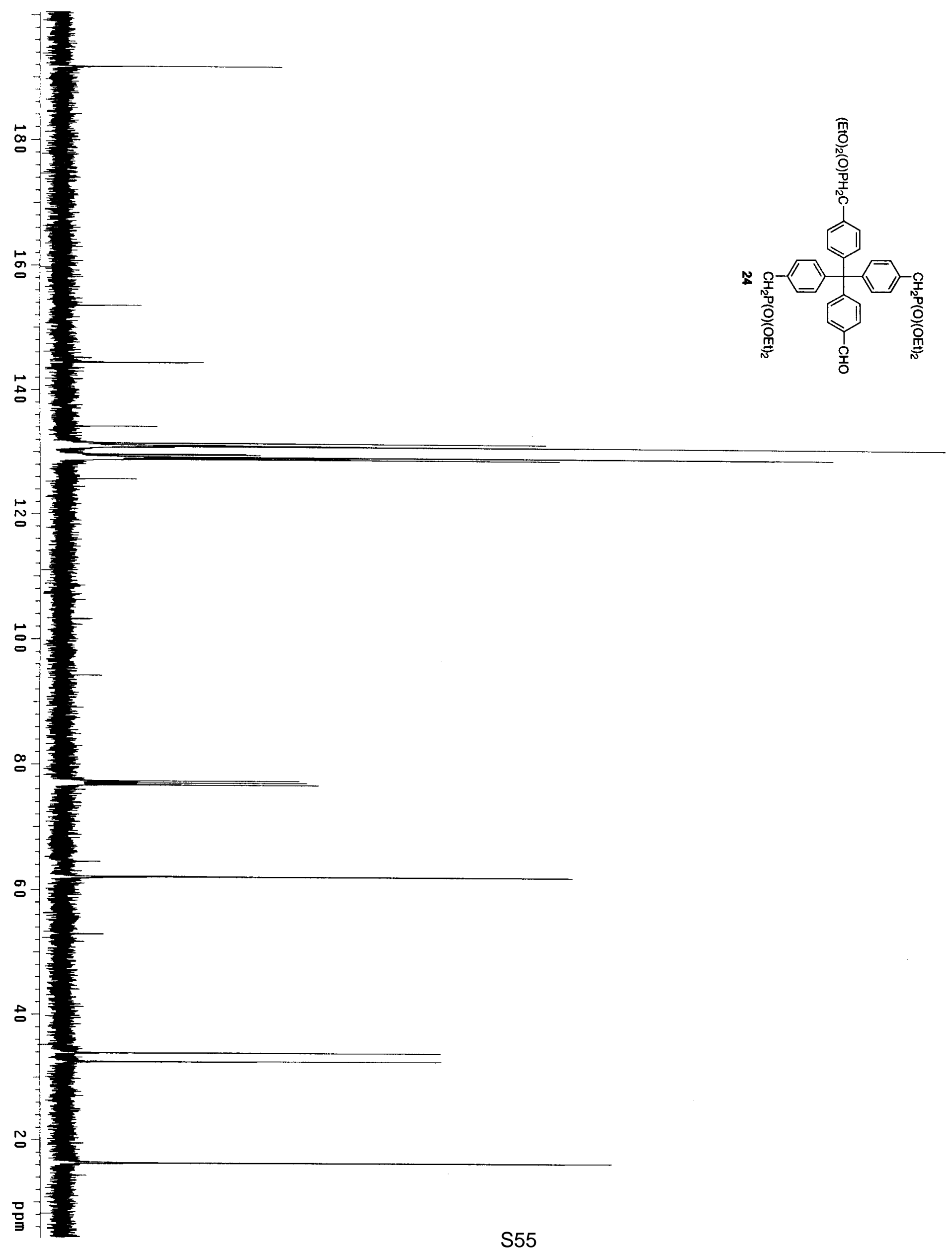


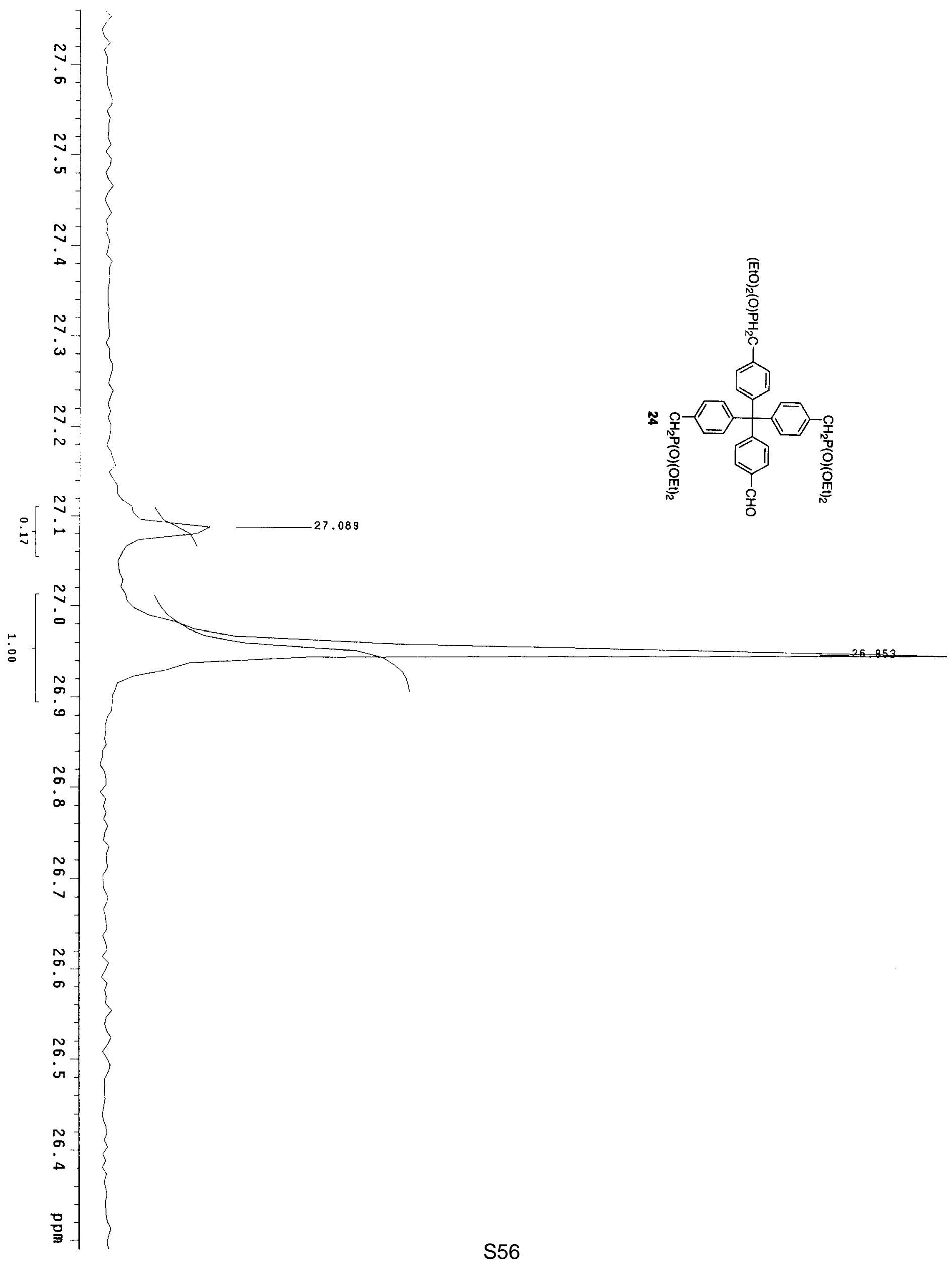



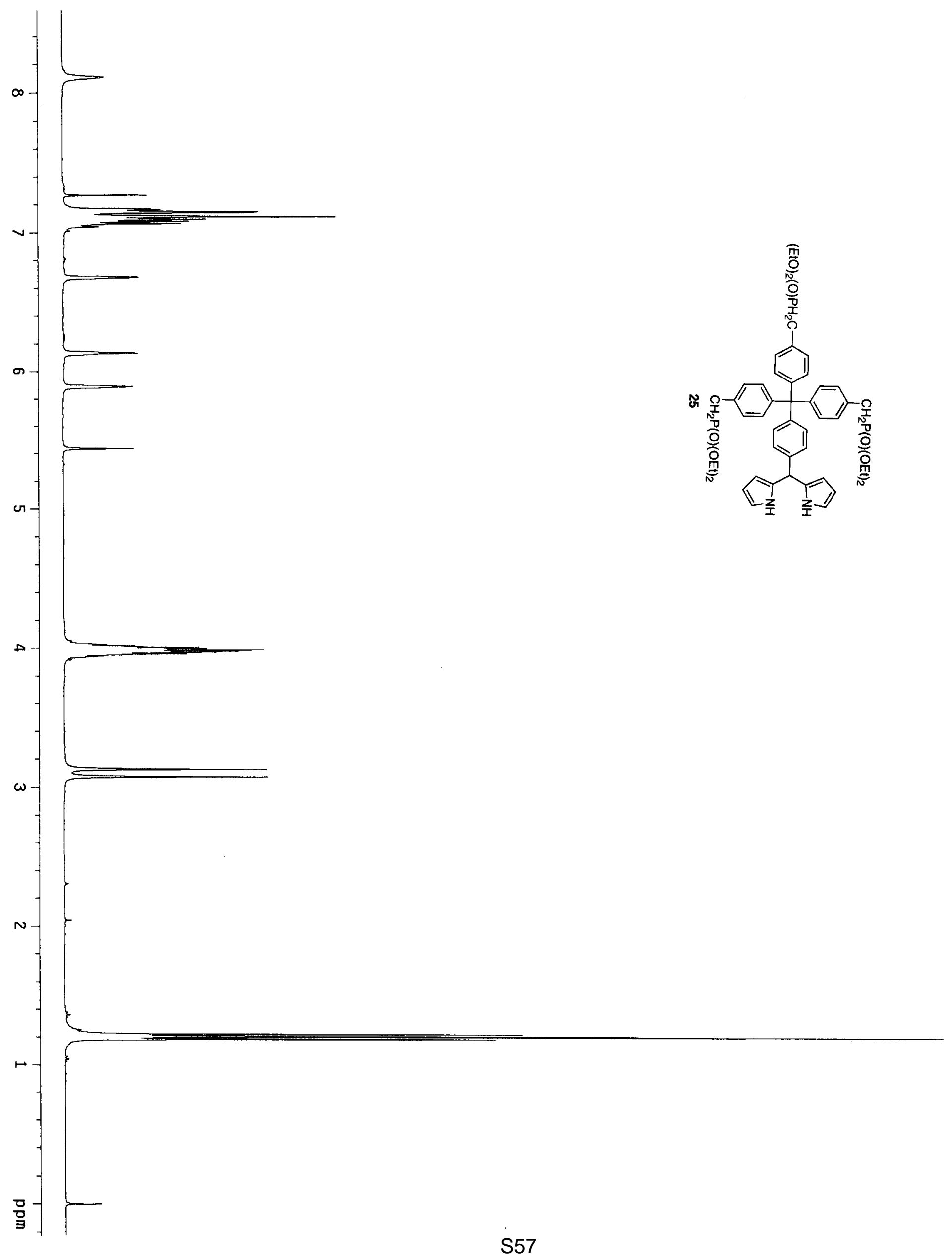


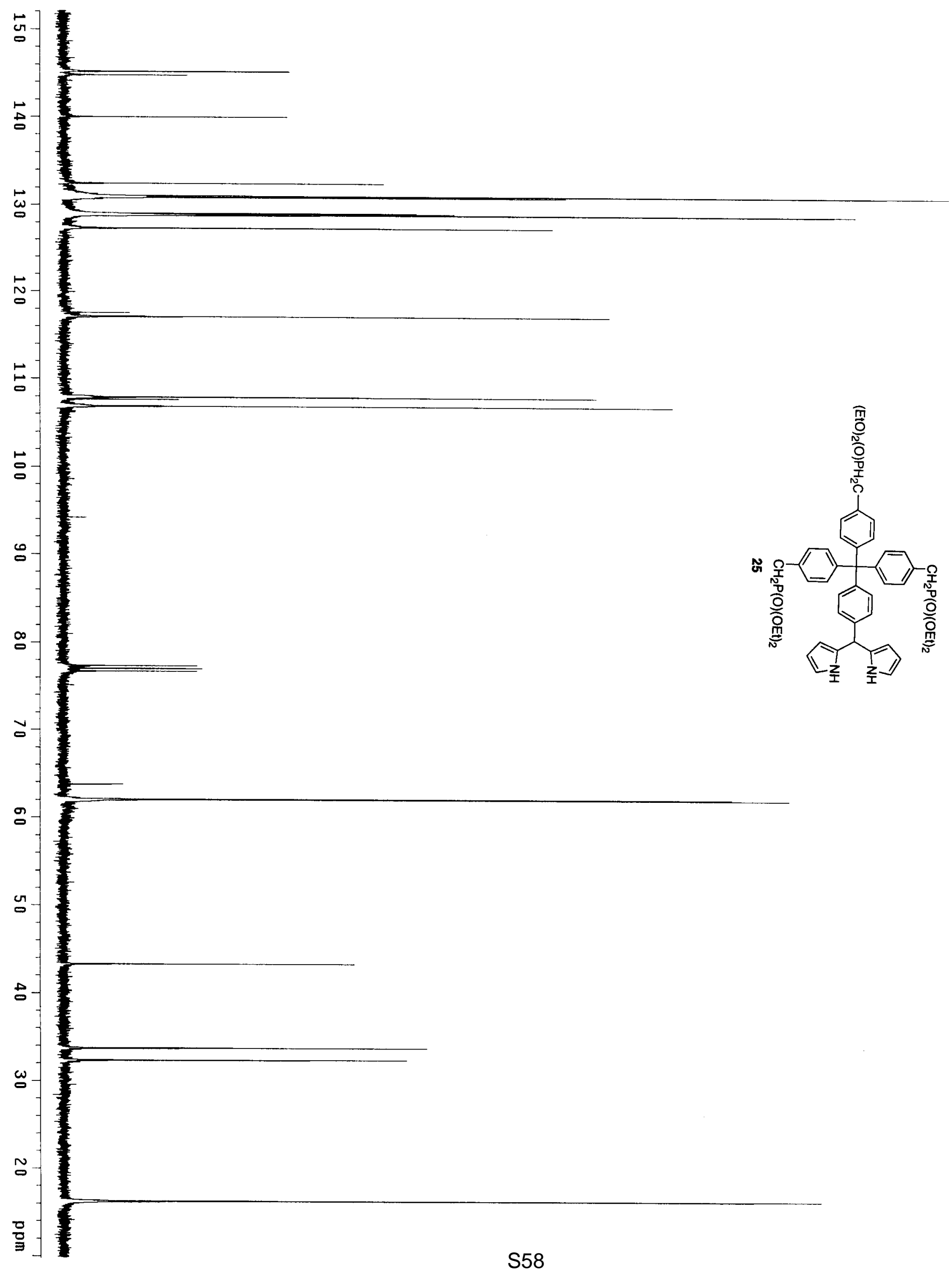




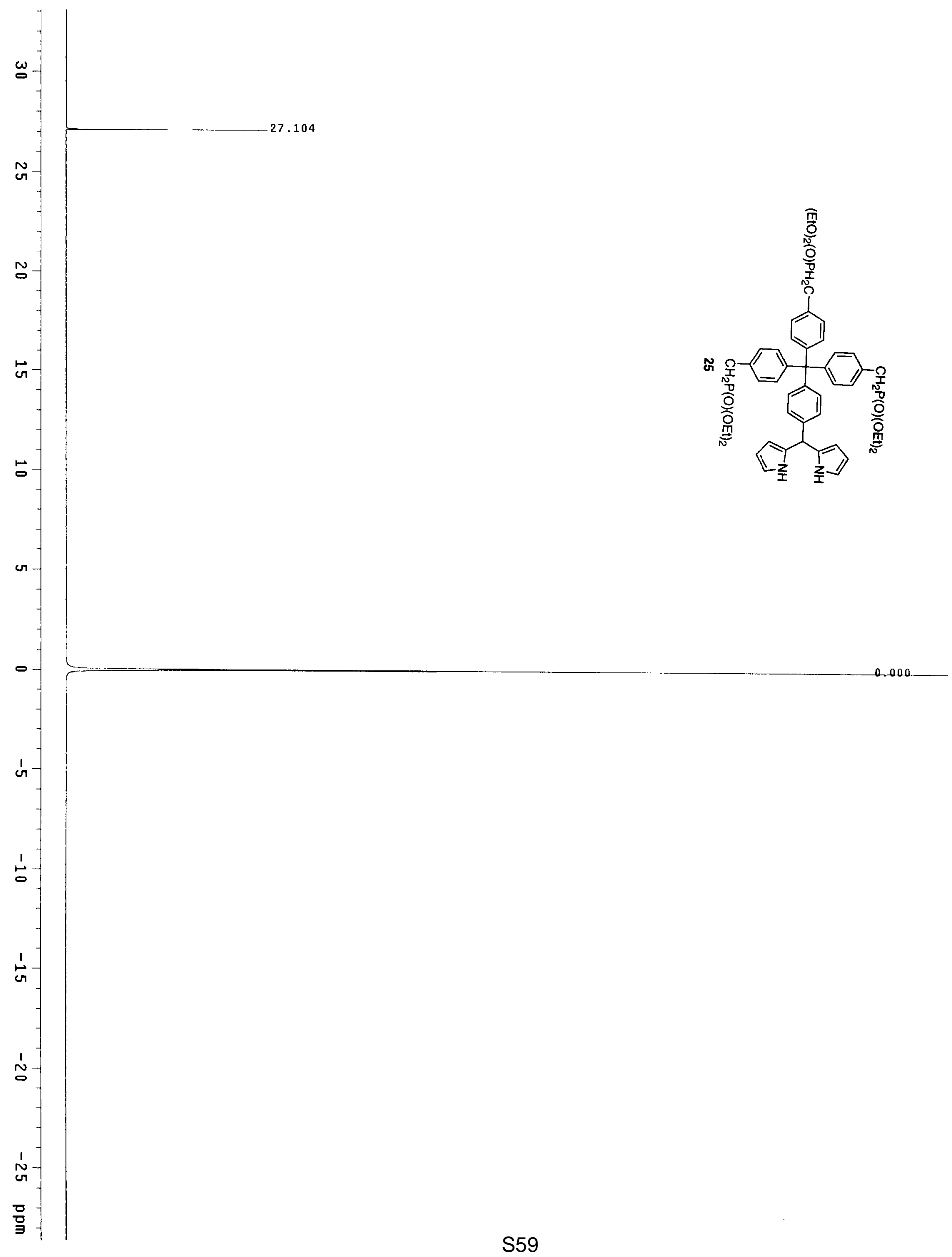




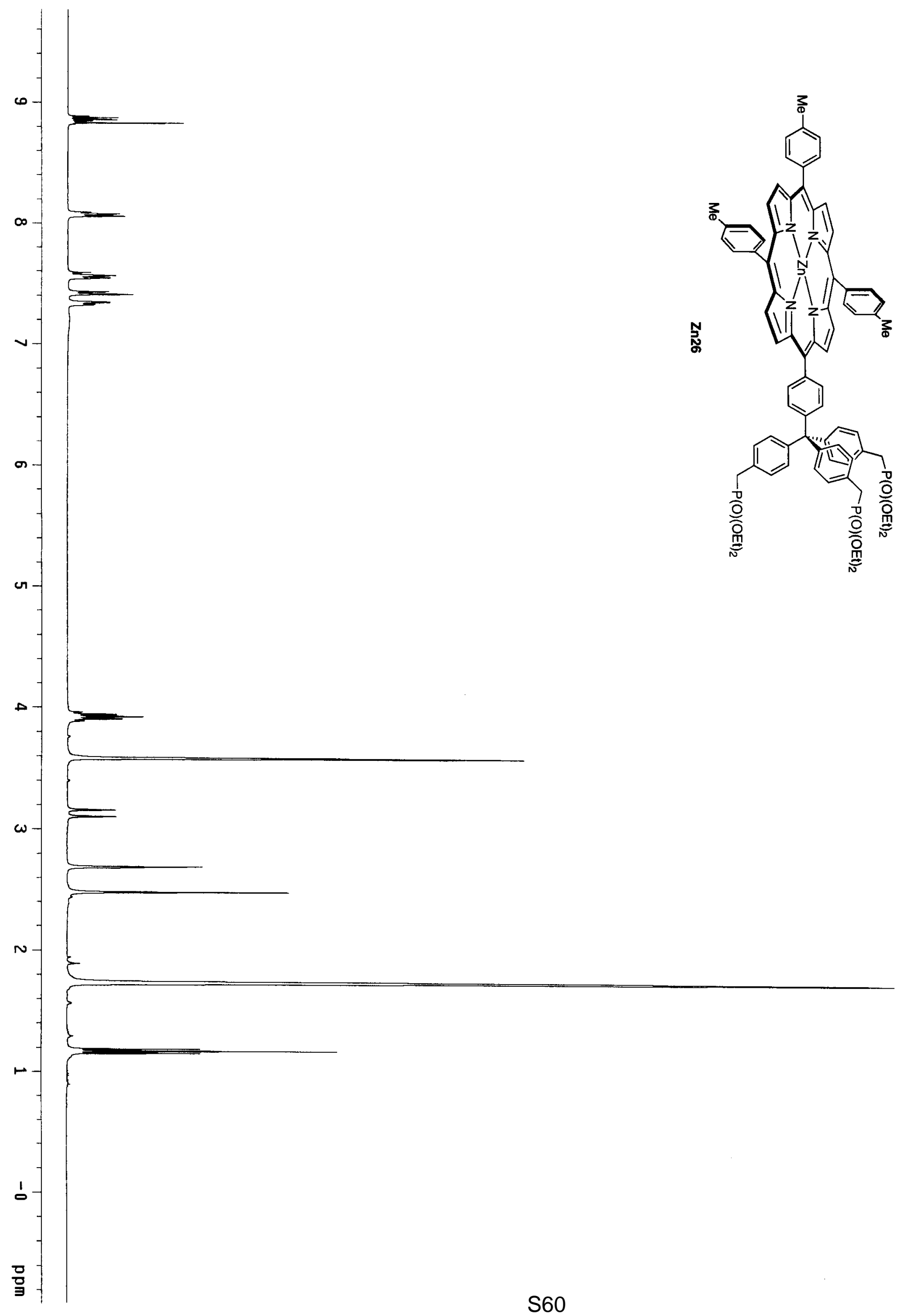




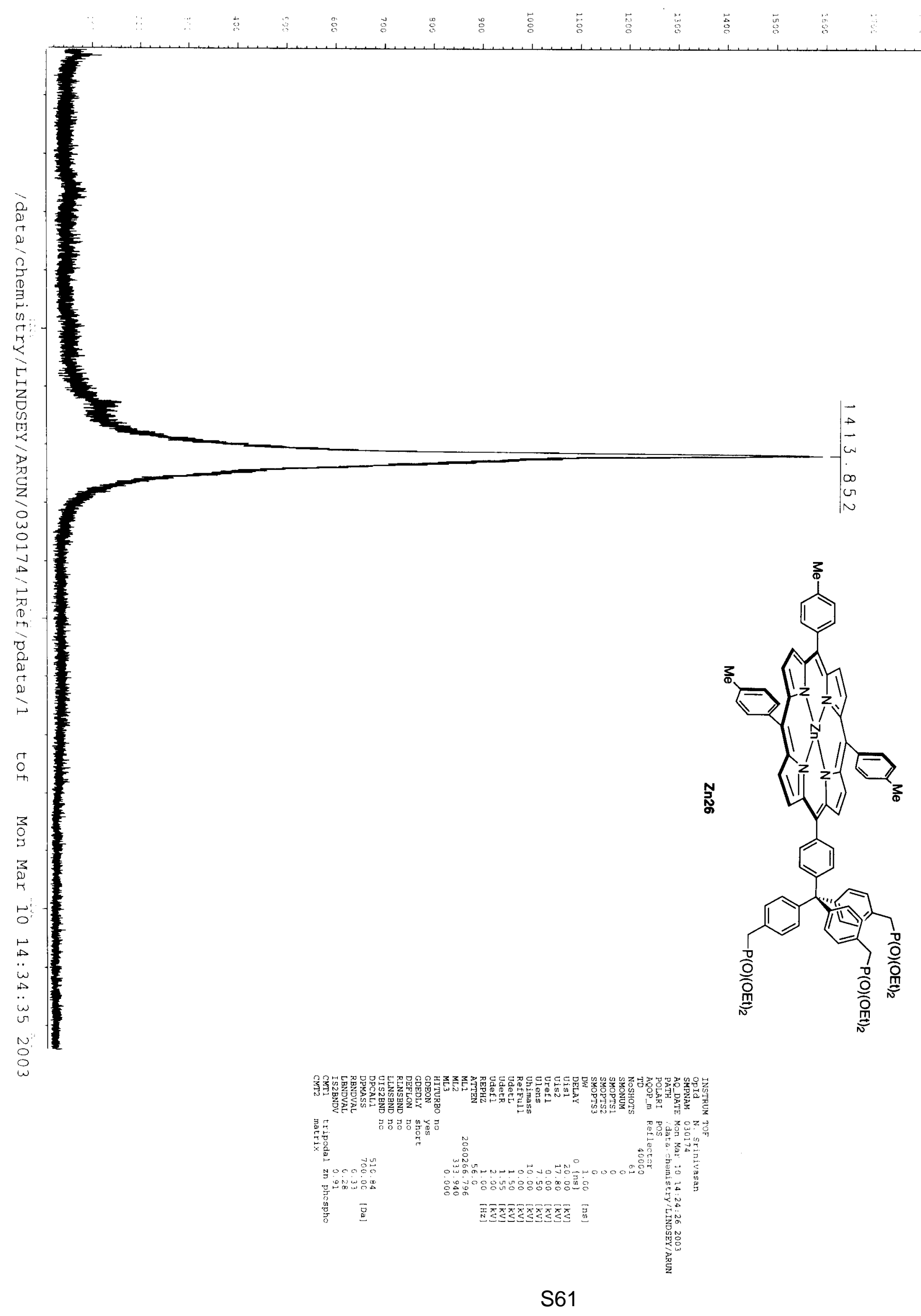




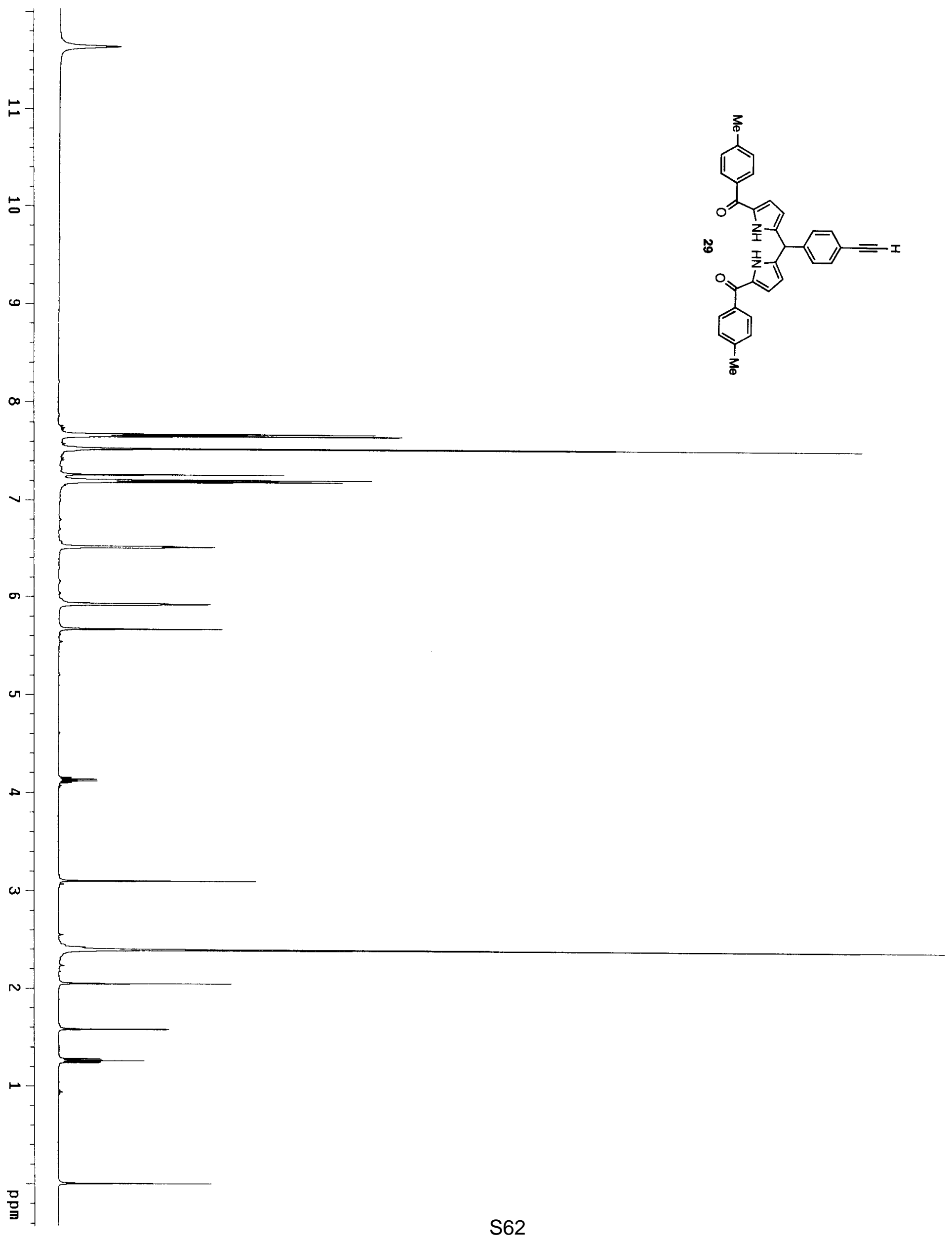



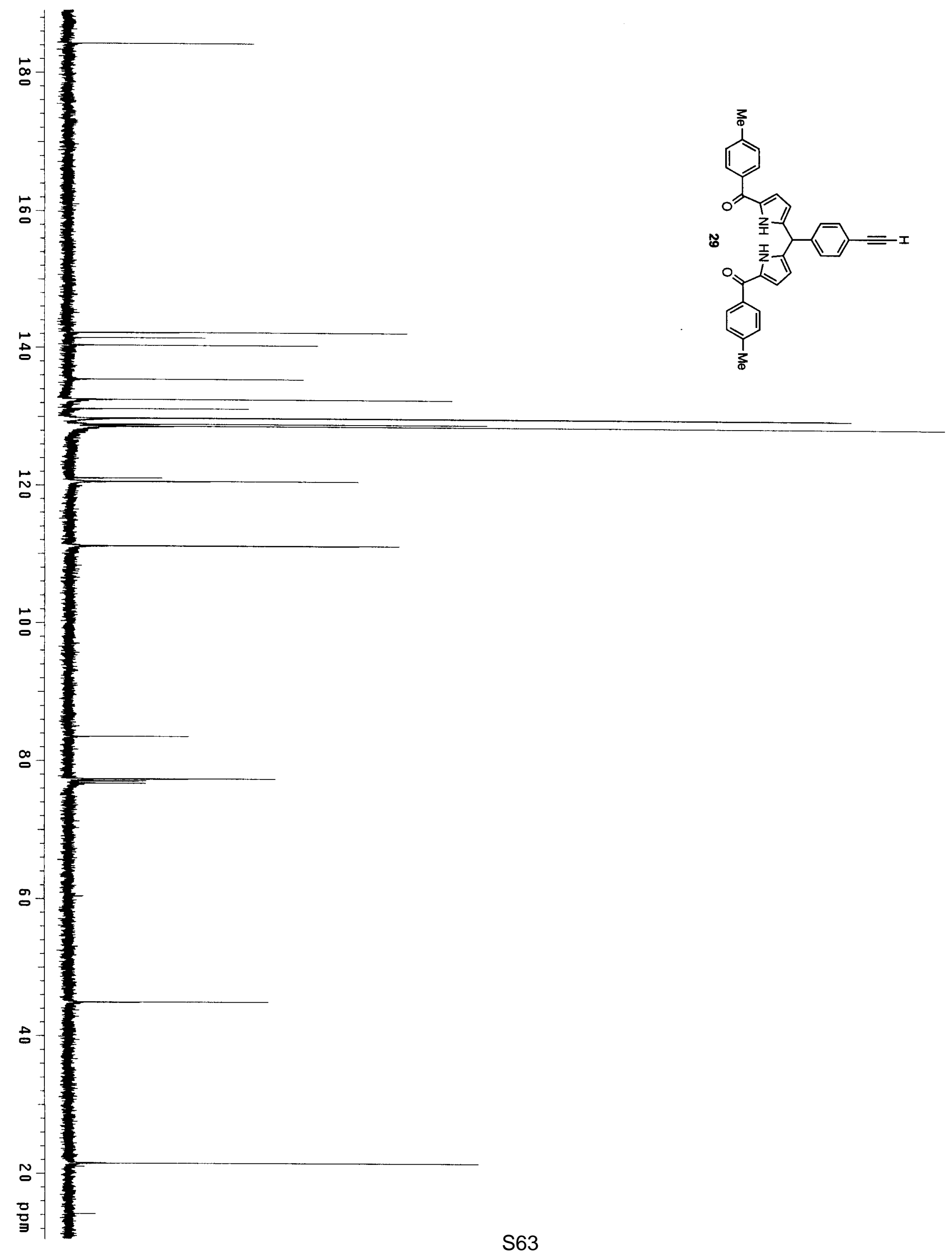


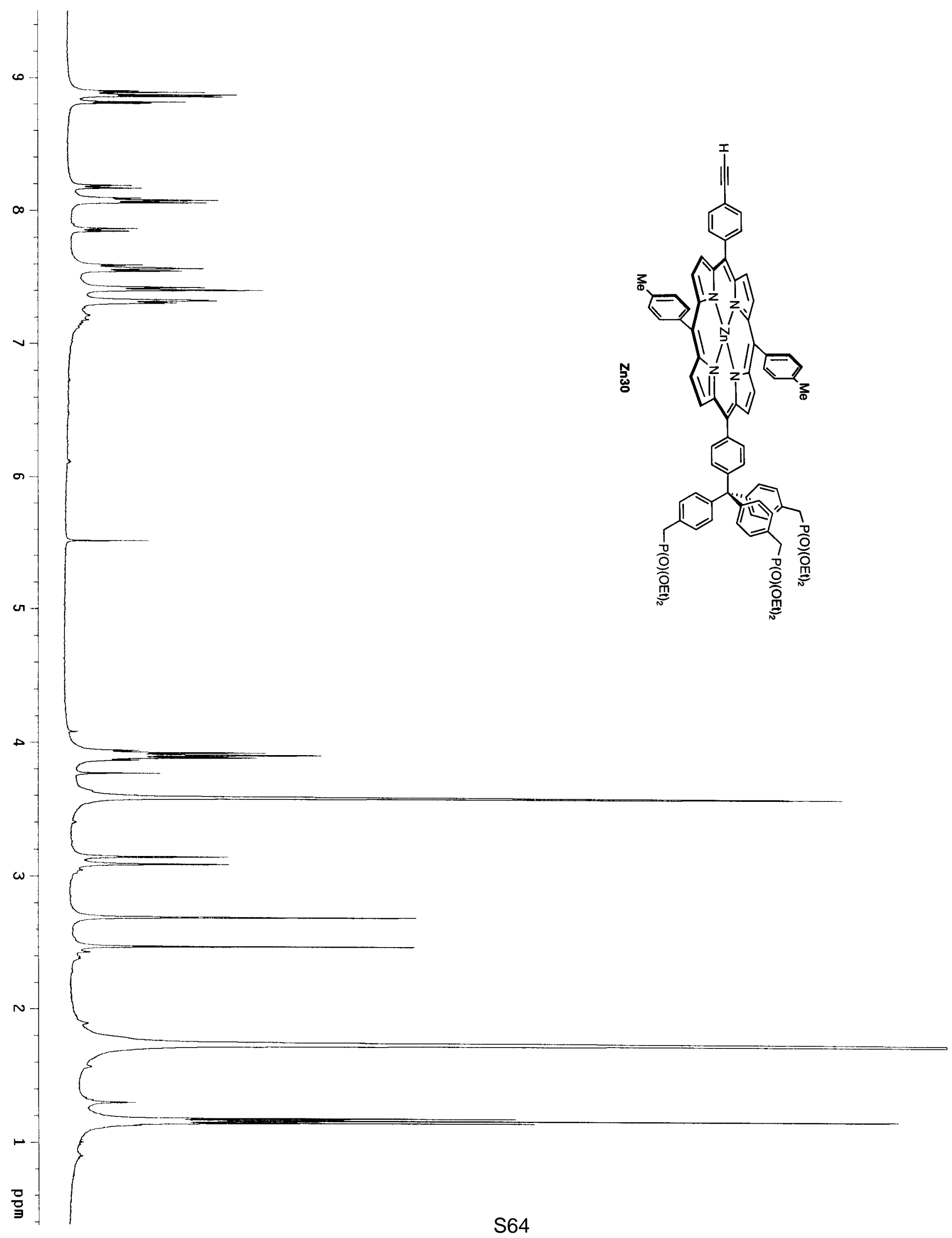




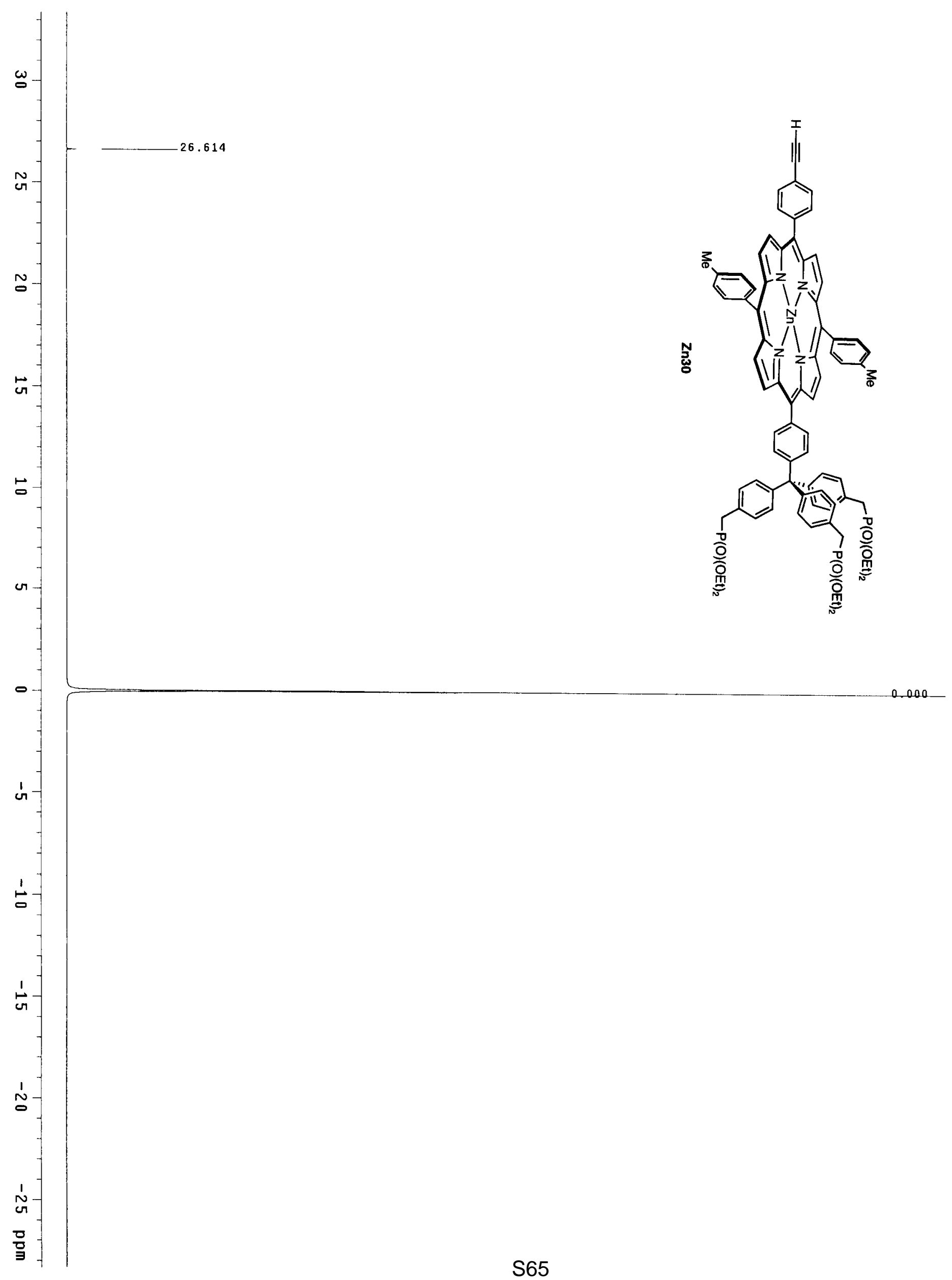




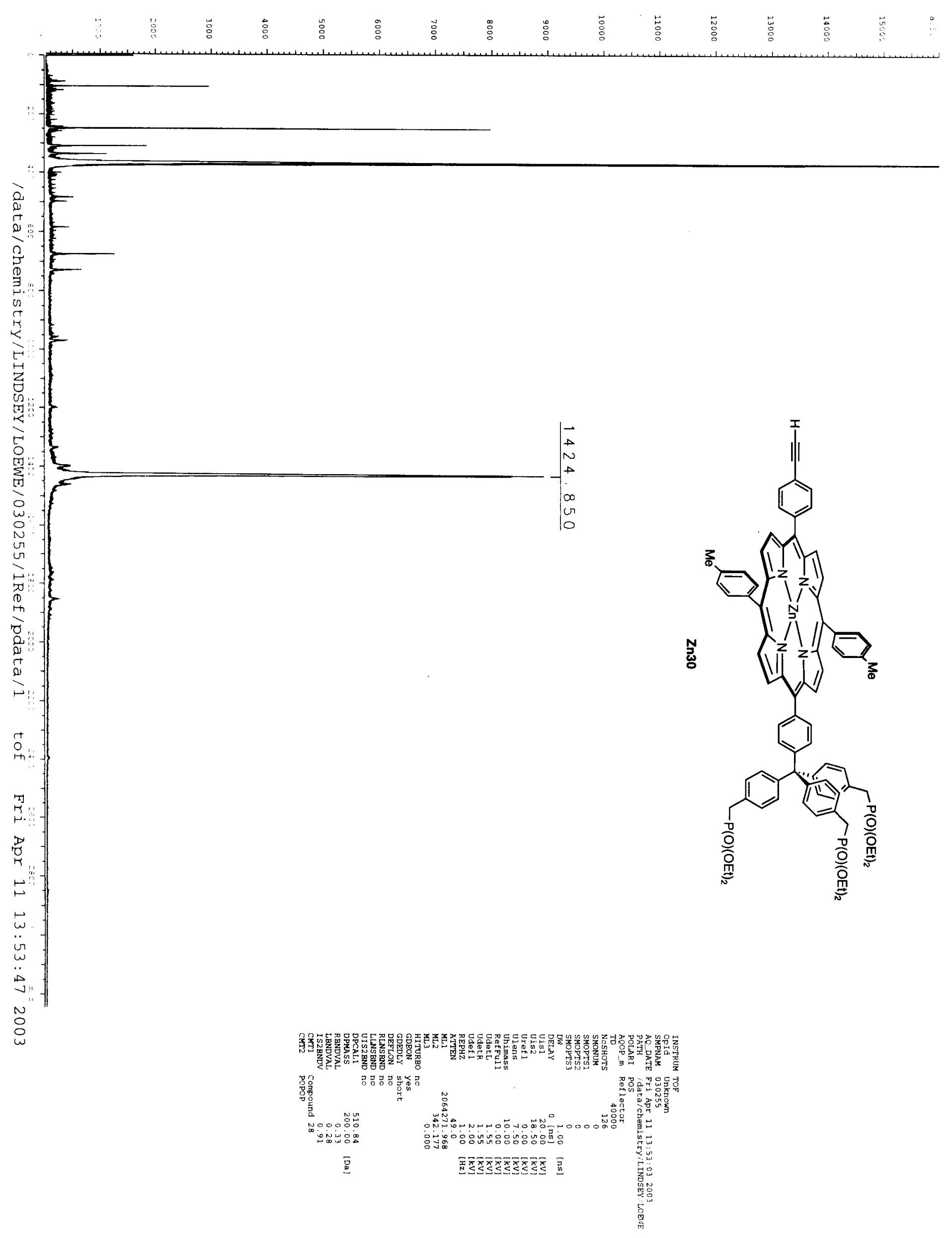

